\title{
Engineered skeletal muscle \\ from human pluripotent stem cells \\ to model muscle disease and regeneration
}

\author{
Doctoral Thesis
}

In partial fulfillment of the requirements for the degree

"Doctor rerum naturalium (Dr. rer. nat.)"

in the Molecular Medicine Study Program

at the Georg-August University Göttingen

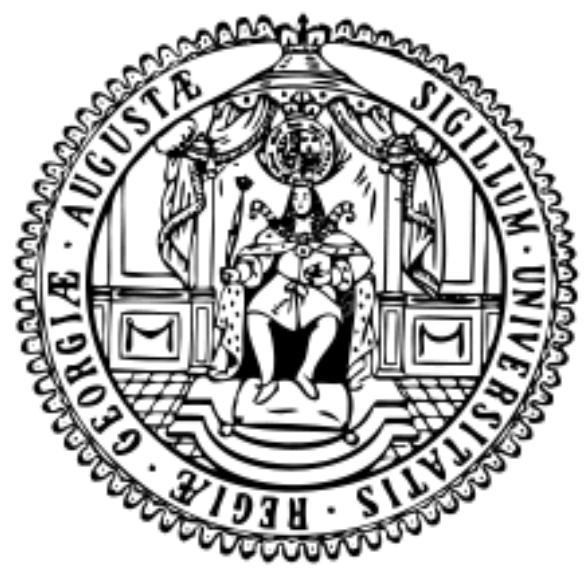

Submitted By

Mina Shahriyari

Born in Mashhad, Iran

Göttingen, December 2020 


\section{Thesis Committee Members}

Dr. med. Malte Tiburcy (Supervisor)

Email: $\quad$ m.tiburcy@med.uni-goettingen.de

Phone: $\quad+49(0) 5513920729$

Postal Address: Institute of Pharmacology and Toxicology

University Medical Center Göttingen

Georg-August University Göttingen

Robert-Koch-Str. 40

37075 Göttingen, Germany

Prof. Dr. med. Wolfram-Hubertus Zimmermann (Supervisor, First thesis committee member)

Email: $\quad$ w.zimmermann@med.uni-goettingen.de

Phone: $\quad+49$ (0) 551395781

Postal Address: Institute of Pharmacology and Toxicology

University Medical Center Göttingen

Georg-August University Göttingen

Robert-Koch-Str. 40

37075 Göttingen, Germany

Prof. Dr. Wolfgang A. Linke (Second thesis committee member)

$\begin{array}{ll}\text { Email: } & \text { wlinke@uni-muenster.de } \\ \text { Phone: } & +49(0) 2518355540 \\ \text { Postal Address: } & \text { Institute of Physiology II } \\ & \text { University Medical Center Münster } \\ & \text { Robert-Koch-Str. 27b } \\ & \text { 48149 Münster, Germany }\end{array}$

Prof. Dr. Andre Fischer (Third thesis committee member)

Email: $\quad$ A.Fischer@med.eni-g.de

Phone: $\quad$ +49 (0) 5513961211

Postal Address: Department for Epigenetics and Systems Medicine in Neurodegenerative Diseases German Center for Neurodegenerative Diseases (DZNE) Göttingen

Georg-August University Göttingen

Von-Siebold-Str. 3a

37075 Göttingen, Germany 


\title{
Thesis Committee Advisory Board
}

\section{Dr. Antje Ebert}

Email: $\quad$ antje.ebert@,med.uni-goettingen.de

Phone: $\quad+49(0) 5513966309$

Postal Address: Department of Cardiology and Pneumology

University Medical Center Göttingen

Georg-August University Göttingen

Robert-Koch-Str. 40

37075 Göttingen, Germany

\section{Dr. Sven Thoms}

\author{
Email: $\quad$ sven.thoms@med.uni-goettingen.de \\ Phone: $\quad$ +49 (0) 5513966261 \\ Postal Address: Department of Child and Adolescent Health \\ University Medical Center Göttingen \\ Georg-August University Göttingen \\ Robert-Koch-Str. 40 \\ 37075 Göttingen, Germany
}

\section{Prof. Dr. med. Bernd Wollnik}

Email: $\quad$ bernd.wollnik@med.uni-goettingen.de

Phone: $\quad+49$ (0) 5513967589

Postal Address: Institute of Human Genetics

University Medical Center Göttingen

Georg-August University Göttingen

Heinrich-Düker-Weg 12

37073 Göttingen, Germany 


\section{Affidavit}

Here I declare that my doctoral thesis entitled

"Engineered skeletal muscle from human pluripotent stem cells to model muscle disease and regeneration"

has been written independently with no other sources and aids than quoted. 


\section{List of Scientific Contributions}

\section{Oral presentations}

Mina, Shahriyari, Wolfram-Hubertus Zimmermann, Malte Tiburcy. Engineered human skeletal muscle from pluripotent stem cell-derived myoblasts - a novel pharmacological tool. $3^{\text {rd }}$ German Pharm-Tox Summit (DGPT) 2018, Göttingen, Germany.

Mina Shahriyari, Chengzu Long, Eric N. Olson, Wolfram-Hubertus Zimmermann, Malte Tiburcy. Human skeletal muscle from induced pluripotent stem cells for the investigation of genetic therapeutic approaches for Duchenne muscular dystrophy. 25 th Congress of German Society for Muscle Diseases (DGM) 2019, Göttingen, Germany.

\section{Poster}

Mina Shahriyari, Chengzu Long, Andreas Unger, Wolfgang A. Linke, Eric N. Olson, WolframHubertus Zimmermann, Malte Tiburcy. Human Engineered Skeletal Muscle from Pluripotent Stem Cells to Phenotype Genetic Therapies of Duchenne Muscular Dystrophy. Myogenesis Gordon Research Conference 2019, Renaissance Tuscany, II Ciocco, Italy.

\section{Patent}

Shahriyari M, Tiburcy M, Zimmermann WH (2020). Herstellung von Skelettmuskelzellen und Skelettmuskelgewebe aus pluripotenten Stammzellen. DE 102019127604.7 (Priority date: 14 October 2019); PCT/EP2020/078738 


\section{Table of Contents}

Acknowledgements .......................................................................................................................................8

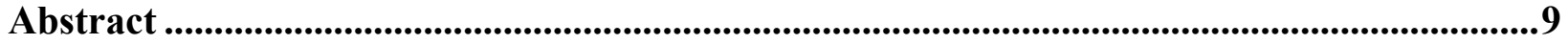

List of figures .............................................................................................................................................10

List of tables .......................................................................................................................................12

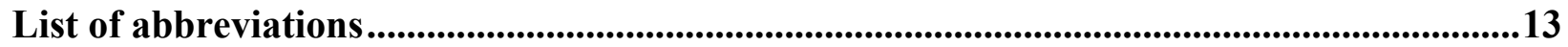

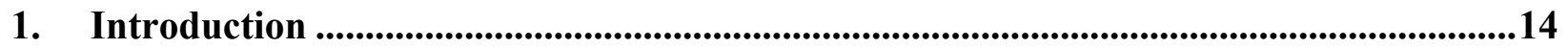

$1.1 \quad$ Structure of skeletal muscle................................................................................15

$1.2 \quad$ Skeletal muscle development in vivo................................................................................16

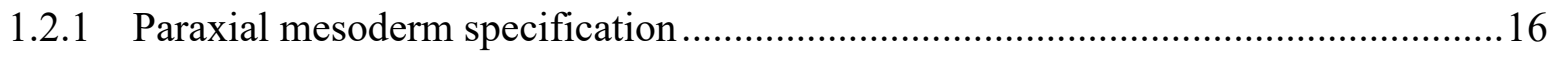

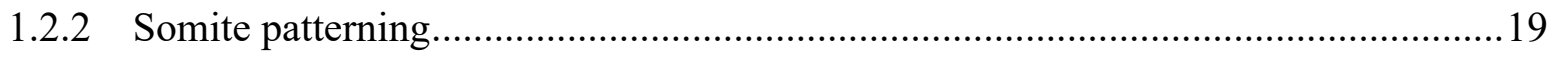

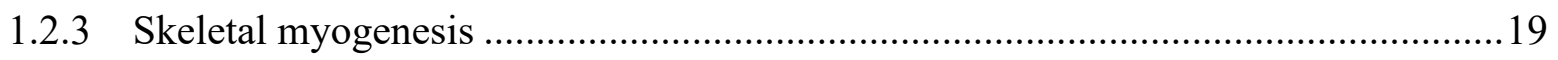

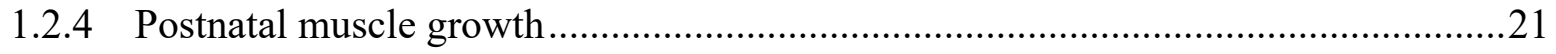

1.3 Contractile function of skeletal muscle ...............................................................22

$1.4 \quad$ Skeletal muscle regeneration .......................................................................................23

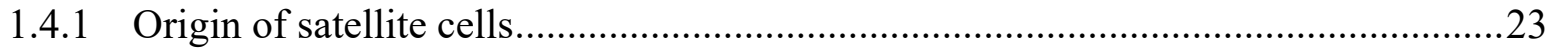

1.4.2 Sequential stages of skeletal muscle regeneration.................................................2.

1.5 Human in vitro models of skeletal muscle..................................................................25

1.5.1 Directed skeletal myocyte differentiation of human pluripotent stem cells ..............25

1.5.2 Tissue engineering of skeletal muscle ..................................................................26

1.5.3 Duchenne muscular dystrophy in in vitro models of human skeletal muscle ...........28

$1.6 \quad$ Aim and hypotheses of the study................................................................................29

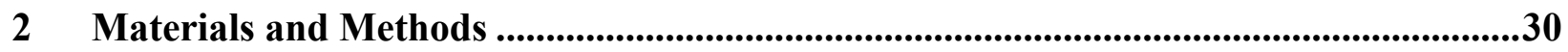

2.1 Human cell culture techniques .................................................................................30

2.1.1 Human pluripotent stem cell lines ..................................................................... 30

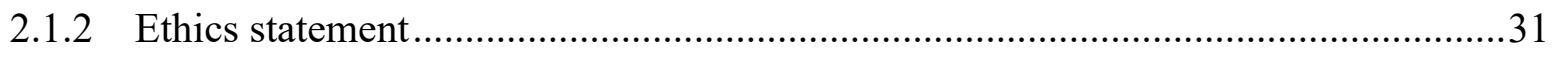

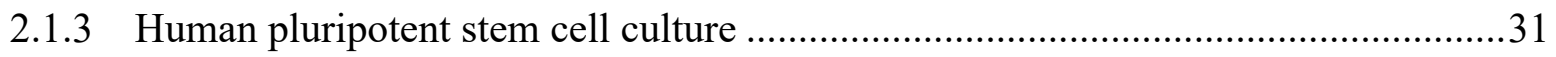


2.1.4 Skeletal muscle differentiation media and reagents ................................................. 31

2.1.5 Directed differentiation of human PSCs into skeletal myocytes .................................34

2.1.6 Enzymatic dissociation of human PSC-derived skeletal myocytes..............................35

2.1.7 Cryopreservation of human PSC-derived skeletal myocytes .....................................35

2.1.8 Thawing of human PSC-derived skeletal myocytes....................................................36

2.2 Human engineered skeletal muscle ..................................................................................36

2.2.1 Preparation of casting molds and static stretchers...................................................

2.2.2 Generation of human bioengineered skeletal muscle (BSM) organoid.......................36

2.2.3 Generation of human engineered skeletal muscle (ESM) ..........................................37

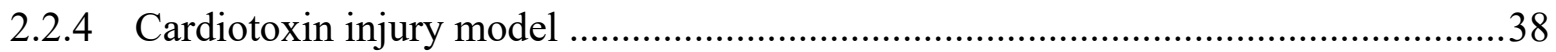

2.3 Immunostaining and confocal imaging ...................................................................39

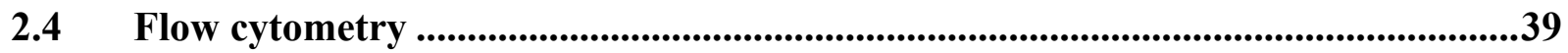

2.5 RNA sequencing.....................................................................................................40

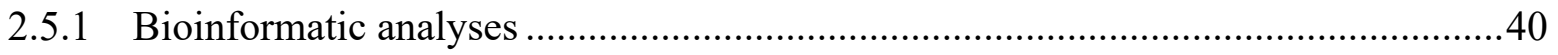

2.6 Single cell transcriptomics by single nuclei RNA sequencing ....................................41

2.6.1 Bioinformatic analysis of single-nucleus RNA-sequencing.....................................42

2.7 Isometric force measurements.........................................................................................43

2.8 Western blot analysis .......................................................................................................43

2.9 Transmission Electron Microscopy ….........................................................................44

$2.10 \quad$ Statistical analysis............................................................................................................

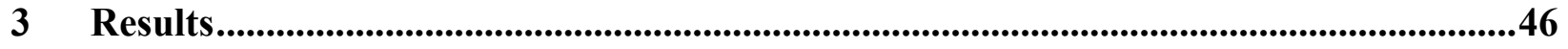

3.1 Directed differentiation of hPSCs into skeletal myocytes...........................................46

3.1.1 Sequential recapitulation of key stages of skeletal muscle development..................46

3.1.2 Evaluation of skeletal muscle differentiation efficiency .........................................51

3.1.3 Transcriptome profiling of skeletal muscle differentiation from hPSCs...................53

3.1.4 Composition of skeletal myocyte cultures on single cell level ................................59

3.2 Human engineered skeletal muscle ................................................................................61

3.2.1 Generation of human bioengineered skeletal muscle organoid from iPSC ................61 
3.2.2 Generation of engineered skeletal muscle with organotypic function .63

3.2.3 Advanced muscle structure of engineered skeletal muscle .........................................66

3.2.4 Creatine supplementation enhances force of ESM....................................................68

3.2.5 Maturation of myosin isoforms by $\mathrm{T} 3$ treatment.................................................... 70

3.3 Regenerative capacity of engineered skeletal muscle.....................................................74

3.3.1 Satellite cells in engineered skeletal muscle ......................................................... 74

3.3.2 Regenerative capacity of cardiotoxin injured ESM...............................................76

3.4 Modeling Duchenne Muscular Dystrophy in engineered skeletal muscle................78

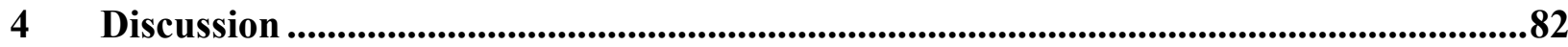

4.1 Robust protocol for skeletal muscle differentiation of human iPSCs.......................83

4.2 Engineered models of skeletal muscle from human PSCs..........................................86

4.2.1 Bioengineered skeletal muscle (BSM) organoid from human PSCs.........................8 87

4.2.2 Engineered skeletal muscle from human PSC-derived skeletal muscle cells ............87

4.3 Myosin isoform switch in ESM under Triiodo-L-thyronine .........................................88

4.4 Regenerative potential of ESM in response to cardiotoxin-induced injury ............89

4.5 Modeling Duchenne Muscular Dystrophy in ESM .......................................................90

$5 \quad$ Summary and perspectives.........................................................................................................92

6 Bibliography.............................................................................................................................93

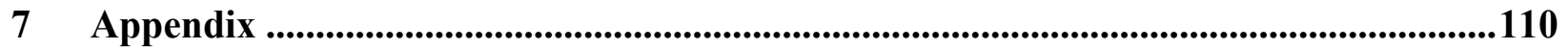

7.1 Reagents...........................................................................................................................110

7.2 Gene panel.......................................................................................................................114

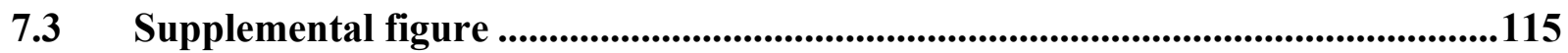




\section{Acknowledgements}

First, I wish to offer my sincerest gratitude to Prof. Wolfram-Hubertus Zimmermann for providing me a golden opportunity to join his research team. He supported me throughout the PhD study with his unique expertise, encouragements and tremendous academic support which altogether made my academic life an enriching experience.

I would like to express my deepest gratitude to my supervisor Dr. Malte Tiburcy, for providing me the chance to complete my studies with this precious project. His professional support and priceless expertise elevated the scientific value of my study. This progression would not have been possible without his patience, his valuable guidance and wholeheartedly contribution to the preparation and completion of the project. I heartily appreciate his trust and kindness as well as his invaluable encouragements.

I would like to express my appreciation to the members of my thesis committee, Prof. Wolfgang A. Linke, and Prof. Andre Fischer, for accepting to evaluate my thesis and whose insightful comments enriched the findings of my study.

My special thanks go to Dr. Patapia Zafeiriou, Dr. Laura Zelarayan, Dr. Tim Meyer and Dr. Angelica Roa for their kind and valuable guidance, availability and inspirational encouragements. I am very grateful to Dr. Elif Levent, Dr. Farah Raad, Ms. Harithaa Anandakumar, Mr. Eric Schoger and Mr. Sebastian Nagel for their generous helps at work and sharing their experiences. I would like to present my appreciation to all the colleagues in the Institute of Pharmacology and Toxicology, specially to the colleagues and lab mates in AG Tiburcy and to the colleagues in human lab, Mrs. Iris Quentin, Mrs. Daria Reher and Mr. Florian Martinpott who with their kind and helpful efforts provided an environment for progression in lab work.

I am specially thankful to my parents and my friend Shaqayeq, who with their cheerful supports don't let me suffer how far they are.

Passing through the long and complicated road to experience a different face of life while completing my studies would have never been possible for me without having my husband Amir, next to me. I can't thank him enough. 
Skeletal muscle disease modeling offers the unique opportunity to investigate devastating muscle diseases like Duchenne Muscle Dystrophy in vitro but requires advanced three-dimensional (3D) model systems reflecting the characteristics of human muscle in vivo. The aim of this study was to generate engineered models of skeletal muscle from human pluripotent stem cells (hPSCs) with physiological properties by recapitulating specific stages of muscle development.

To allow for robust skeletal muscle tissue engineering first a directed differentiation protocol was established in 2D culture under serum-free conditions. Comparison of hPSC differentiation to embryonic muscle development confirmed significant overlap with characteristic signatures of paraxial mesoderm, dermatomyotome, and somite stage. The protocol robustly directed multiple hPSC lines into skeletal muscle cells in 2D culture as well as in a collagen-1/Matrigel ${ }^{\circledR}$ hydrogel in 3D generating bioengineered skeletal muscle (BSM) organoid. By identifying additional maturation cues (creatine, triiod-L-thyronine) hPSC-derived skeletal myogenic cells embedded into a collagen-1/Matrigel ${ }^{\circledR}$ hydrogel generated engineered skeletal muscle (ESM) with compact muscle syncytia, anisotropically arranged sarcomeres, properly localized dystrophin-associated complex proteins, and contractile function of developing fast muscle. Importantly, Pax7-positive cells were found adjacent to muscle fibers underneath a laminin-positive basal lamina in a satellitelike cell position. Cardiotoxin injury of ESM induced a regenerative response with recovery of tetanic force after complete loss of function. Finally, modelling of Duchenne Muscular Dystrophy (DMD) in ESM demonstrates "proof of concept" for efficacy of CRISPR/Cas9 based exon skipping.

Collectively, human BSM and ESM models provide unprecedented opportunities to study muscle development, maturation, and regeneration in vitro and may serve as preclinical test bed for novel therapies of skeletal muscle disease. 


\section{List of figures}

Figure 1. The three types of muscle tissue in the human body. .............................................. 14

Figure 2. The hierarchical structure of skeletal muscle...................................................... 15

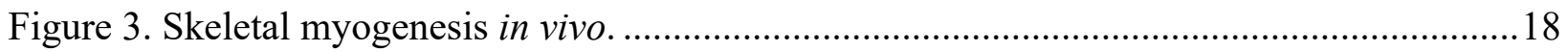

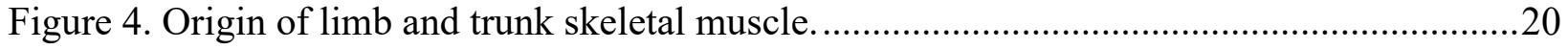

Figure 5. Force trace patterns of mammalian muscle fibers....................................................23

Figure 6. Satellite cell niche of skeletal muscle. ...................................................................24

Figure 7. Tissue engineering models of skeletal muscle from hPSCs. ...................................22

Figure 8. Directed limb skeletal myogenesis in vitro................................................................48

Figure 9. Recapitulation of sequential distinct phases of myogenesis during directed skeletal muscle differentiation from human PSCs.

Figure 10. Development of key marker proteins during directed skeletal muscle differentiation of hPSCs. .50

Figure 11. Efficient differentiation of hPSCs into skeletal muscle cell populations...................51

Figure 12. Robust skeletal muscle differentiation from multiple hPSCs lines..........................52

Figure 13. Unbiased transcriptome profiling identifies temporally distinct clusters of skeletal muscle development.

Figure 14. Signature gene expression in temporal coexpression clusters.

Figure 15. Coexpression clusters in vitro overlap with embryonic muscle development in vivo. 57

Figure 16. Identification of skeletal muscle maturation genes in coexpression cluster. .59

Figure 17. Single cell transcriptomes of skeletal muscle differentiation. 60

Figure 18. Generation of functional human bioengineered skeletal muscle (BSM) organoid from PSCs.

Figure 19. Generation of highly organized skeletal myofibrils in human bioengineered skeletal muscle.

Figure 20. Contractile function of engineered skeletal muscle increases with time.

Figure 21. Advanced development of skeletal muscle structures in human engineered skeletal muscle.

Figure 22. Functional nicotinic acetylcholine receptors in ESM.

Figure 23. Ultrastructure of human engineered skeletal muscle. 
Figure 24. Creatine supplementation increases ESM twitch tension.

Figure 25. Effect of thyroid hormone on twitch tension generated by ESM. .............................70

Figure 26. Advanced contractile kinetics of ESM by thyroid hormone treatment.....................71

Figure 27. Thyroid hormone elevates the tetanus threshold of human ESM. .......................... 72

Figure 28. Myosin heavy chain isoform expression in human ESM treated with thyroid hormone.

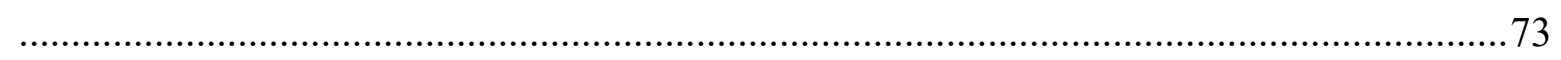

Figure 29. PAX7-positive cells adopt a satellite cell niche in human ESM.............................75

Figure 30. Functional regeneration of human engineered skeletal muscle. ................................77

Figure 31. Efficient generation of skeletal myocytes from DMD and myoedited DMD iPSC

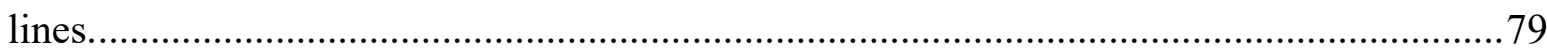

Figure 32. Restoration of Dystrophin expression in DMD ESM by myoediting........................80

Figure 33. Rescue of DMD ESM dysfunction by myoediting. .............................................. 81

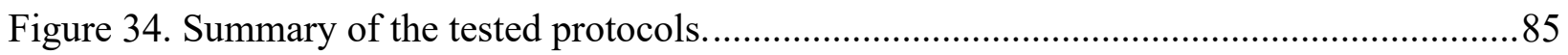




\section{List of tables}

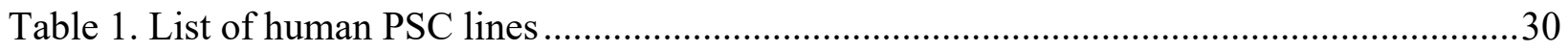

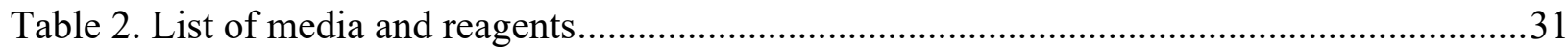

Table 3. Composition of hydrogel master mix for BSM ............................................................ 37

Table 4. Composition of hydrogel master mix for ESM ........................................................... 38

Table 5. Annotation of biological processes to coexpression clusters ............................................56 


\section{List of abbreviations}

$\begin{array}{ll}\text { aPSM } & \text { anterior Pre Somitic Mesoderm } \\ \text { ACh } & \text { AcetylCholine } \\ \text { AChR } & \text { AcetylCholine Receptors } \\ \text { BMP } & \text { Bone Morphogenic Protein } \\ \text { BSM } & \text { Bioengineered Skeletal Muscle } \\ \text { CRISPR } & \text { Clustered Regularly Interspaced Short Palindromic Repeats } \\ \text { Ctrl } & \text { Control } \\ \text { CTX } & \text { Cardiotoxin } \\ \text { DMD } & \text { Duchenne Muscular Dystrophy } \\ \text { DMEM } & \text { Dulbecco's Modified Eagle Medium } \\ \text { ECM } & \text { Extra Cellular Matrix } \\ \text { EHM } & \text { Engineered Heart Muscle } \\ \text { ESC } & \text { Embryonic Stem Cells } \\ \text { ESM } & \text { Engineered Skeletal Muscle } \\ \text { FGF } & \text { Fibroblast Growth Factor } \\ \text { HGF } & \text { Hepatocyte Growth Factor } \\ \text { IGF } & \text { Insulin-like Growth Factor } \\ \text { PBS } & \text { Phosphate Buffered Saline } \\ \text { PDMS } & \text { Polydimethylsiloxane } \\ \text { PM } & \text { Paraxial Mesoderm } \\ \text { PSCs } & \text { Pluripotent Stem Cells } \\ \text { PSM } & \text { PreSomitic Mesoderm } \\ \text { pPSM } & \text { posterior PreSomitic Mesoderm } \\ \text { RFD } & \text { Rate of Force Development } \\ \text { RNA } & \text { Ribonucleic Acid } \\ \text { RPKM } & \text { Reads Per Kilobase Million } \\ \text { T3 } & \text { Triiodo-L-thyronine } \\ \text { TEM } & \text { Transmission Electron Microscope } \\ \text { TT } & \text { Twitch Tension } \\ \text { VEGF } & \text { Vascular Endothelial Growth Factor } \\ \text { WT } & \text { Wild Type } \\ & \end{array}$




\section{Introduction}

Skeletal muscle as the largest tissue in the human body comprises more than 600 individual muscles across the body (Janssen et al. 2000) performing essential functions not only in movement, but also in power generation, heat production, homeostasis and metabolism regulation (Ostrovidov et al. 2014). Moreover, the robust regeneration capacity of skeletal muscle allows it to efficiently restore function after traumatic injury (Rosenblatt 1992). Despite the high regenerative potential, many types of diseases including metabolic, neuromuscular and dystrophic disorders can functionally impair skeletal muscle leading to jeopardized quality of life.

Developing a physiological reliable skeletal muscle in vitro from human pluripotent stem cells will provide an invaluable tool to better understand the basis of muscle regeneration as well as effectively model skeletal muscle disease and elucidate potential therapeutic approaches. To achieve this goal, Nature's blueprint of skeletal muscle structure and embryonic muscle development in vivo was studied to closely recapitulate these steps in vitro.

In the human body, three types of muscle are recognized: 1) smooth muscle existing in the wall of the internal organs, 2) cardiac muscle only present in the heart and 3) skeletal muscle distributed through the body and connected to the skeleton (Figure 1). Contrary to smooth and cardiac muscle, skeletal muscle can be moved voluntarily (Frontera and Ochala 2015).

A

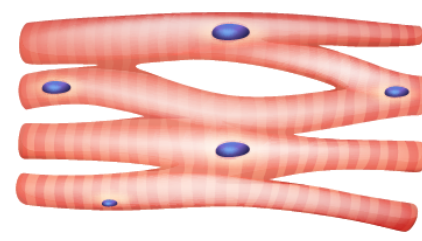

Cardiac muscle
B

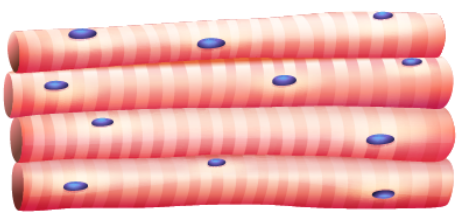

Skeletal muscle

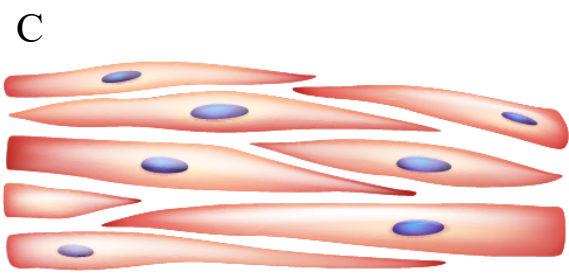

Smooth muscle

Figure 1. The three types of muscle tissue in the human body.

A, Cardiac muscle with branching striated cells. B, Skeletal muscle with single, long striated cells which are multinucleated, and $\mathbf{C}$, Smooth muscle with single fusiform cells without striations. (Image from www.scientistcindy.com) 


\subsection{Structure of skeletal muscle}

Skeletal muscle encompasses a very organized hierarchical structure. Each skeletal muscle is composed of several muscle fascicules surrounded by fibrous perimysium. Each muscle fascicule contains several muscle fibers which are surrounded by endomysium. Each muscle fiber is a multinucleated muscle cell which has a cylindrical shape with a diameter between 50 to $100 \mu \mathrm{m}$ and a length up to $\mathrm{cm}$ scale. The plasma membrane of muscle fibers (sarcolemma) is encased by a laminin- and collagen IV-rich basal lamina (Gillies and Lieber 2011). Skeletal muscle stem cells (satellite cells) reside between sarcolemma and basal lamina adjacent to the muscle fiber. In mature muscle fibers the nuclei are positioned in the periphery just underneath the sarcolemma (Figure 2).

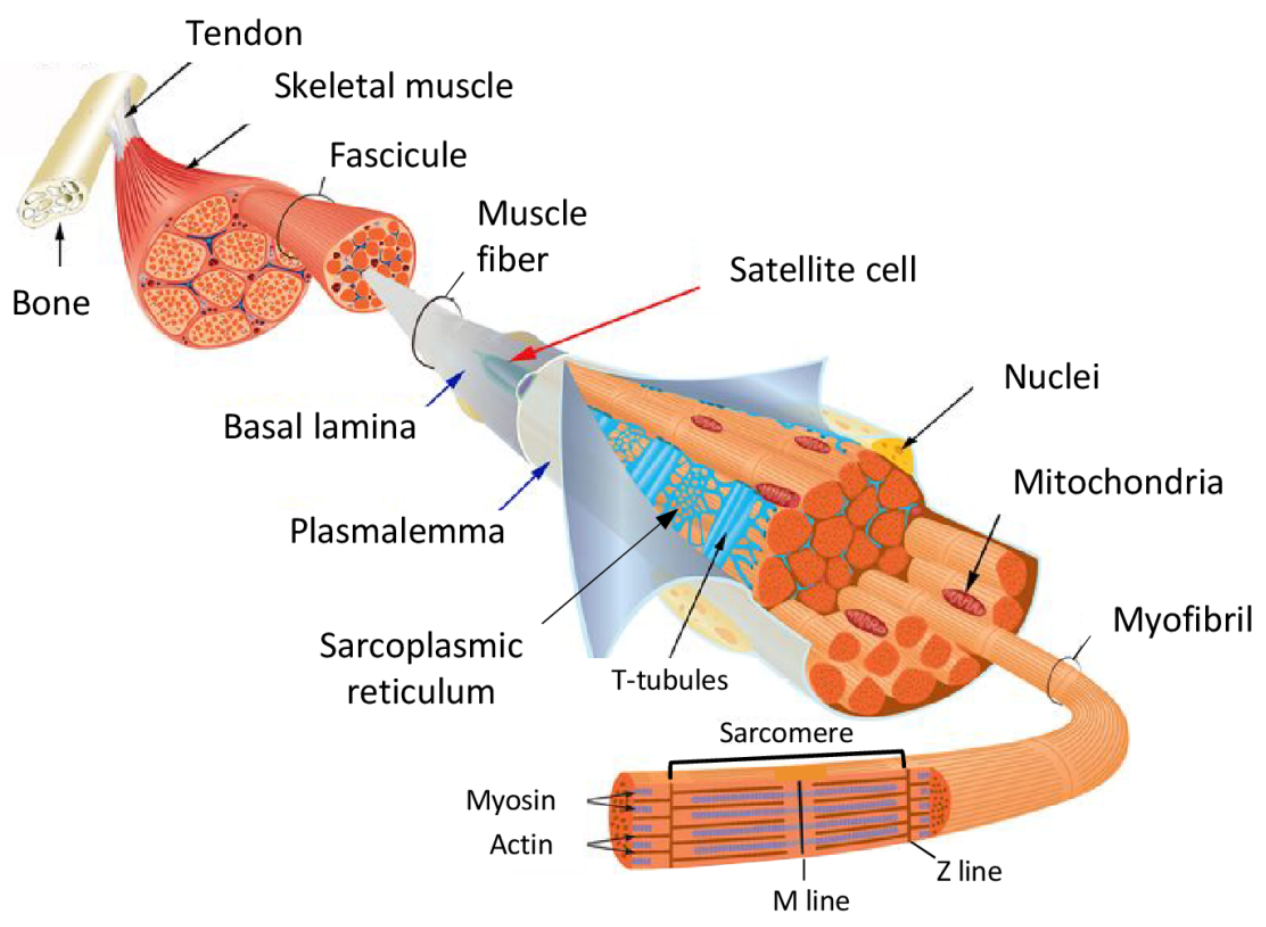

Figure 2. The hierarchical structure of skeletal muscle.

Skeletal muscle attaches to the bone by tendons. Each skeletal muscle is made up of fascicules. Fascicules contain several muscle fibers. Muscle fibers are multinucleated with nuclei located at the periphery of the cell underneath the sarcolemma. The sarcolemma of each muscle fiber is surrounded by a basal lamina. Satellite cells are attached to the muscle fiber between the sarcolemma and basal lamina. Mitochondria and membranous structure of $\mathrm{T}$ tubules/sarcoplasmic reticulum are present in the sarcoplasm. Muscle fibers contain several myofibrils which are composed of repeating sarcomere units (defined by $\mathrm{Z}$ line). In each sarcomere, the $\mathrm{Z}$ line is the anchoring band of the actin filaments and $\mathrm{M}$ line is the anchoring band of the myosin filaments (Image adapted with permission from Development, Company of Biologists, Relaix and Zammit 2012). 
Each muscle fiber contains several myofibrils encircled by T-tubules and sarcoplasmic reticulum (SR) which form close contacts (triads). Mitochondria are located adjacent to SR along the myofibrils. Each myofibril consists of myofilaments, structural and regulatory proteins. Assembly of myofilaments in a highly organized pattern forms the sarcomere with a length of 1.9 to $3 \mu \mathrm{m}$ (Cutts 1988). Each sarcomere is considered as the functional unit of the muscle (Frontera and Ochala 2015). The two most abundant myofilament proteins in the sarcomere are the thin filament actin and the thick filament myosin (Figure 2). Another structural myofilament protein with multifaceted roles in the sarcomere is titin which stabilizes the alignment of myosins (Linke 2018; Swist et al. 2020).

\subsection{Skeletal muscle development in vivo}

Skeletal muscle development is a long, multistep process that starts with paraxial mesoderm formation from pluripotent preimplantation embryo (epiblast) and continues with somite formation, primary skeletal myogenesis and secondary skeletal myogenesis that will be followed by postnatal and adult muscle growth. This process is regulated by a set of specific signaling molecules and transcription factors (Figure 3A,B).

\subsubsection{Paraxial mesoderm specification}

During early gastrulation paraxial mesoderm (PM) forms as bilateral strips of presomitic mesoderm (PSM) flanking the neural tube and notochord alongside the forming posterior-anterior (P-A) axis at the posterior domain of the embryo (Chal and Pourquié 2009).

When Wnt-signaling is activated in multipotent progenitors near the embryonic node, dishevelled (Dvl/Dsh) will be recruited to inhibit the glycogen synthase kinase 3 (GSK3) located in the betacatenin destruction complex. This leads to an accumulation of free non-phosphorylated betacatenin in the cytosol, which will be translocated to the nucleus and activates Wnt-target genes such as $T$ (brachyury) to give rise to primitive streak mesoderm (Liu et al. 1999; Ramkumar and Anderson 2011). In the absence of BMP signaling, the enhancer N1 will be activated by synergistic action of Wnt and FGF signals. N1 is responsible for Sox2 regulation to give rise to T/Sox2 
coexpressing neuromesodermal progenitors (NMP) in paraxial mesoderm (Garriock et al. 2015; Takemoto et al. 2011). WNT together with FGF signaling will repress the neural fate by antagonizing Sox 2 in NMPs and promote the formation of posterior presomitic mesoderm (pPSM) by upregulation of Tbx6 and Msgn1 (Takemoto et al. 2011; Yoon, Moon, and Wold 2000). Oscillations of the segmentation clock which generates pulses of Wnt, FGF and Notch signaling conduct the differentiation of pPSM towards the somite (Aulehla and Pourquié 2008; Dunty et al. 2008; Hubaud and Pourquié 2014; Miura et al. 2006). A decreasing posterior to anterior (P-A) gradient of Wnt/FGF activity with simultaneous activation of Notch signaling is proposed as defined threshold called determination front. At this level Tbx6 in pPSM will respond to the segmentation clock by activating the expression of segmentation genes such as Hes 7 and Mesp2 to enter the anterior presomitic mesoderm (aPSM) and express genes such as Foxc2, Meox1 and Pax3 (González et al. 2013; Kume et al. 2001; Mankoo et al. 2003; Oginuma et al. 2008; Pourquié 2011; Saga et al. 1997). 


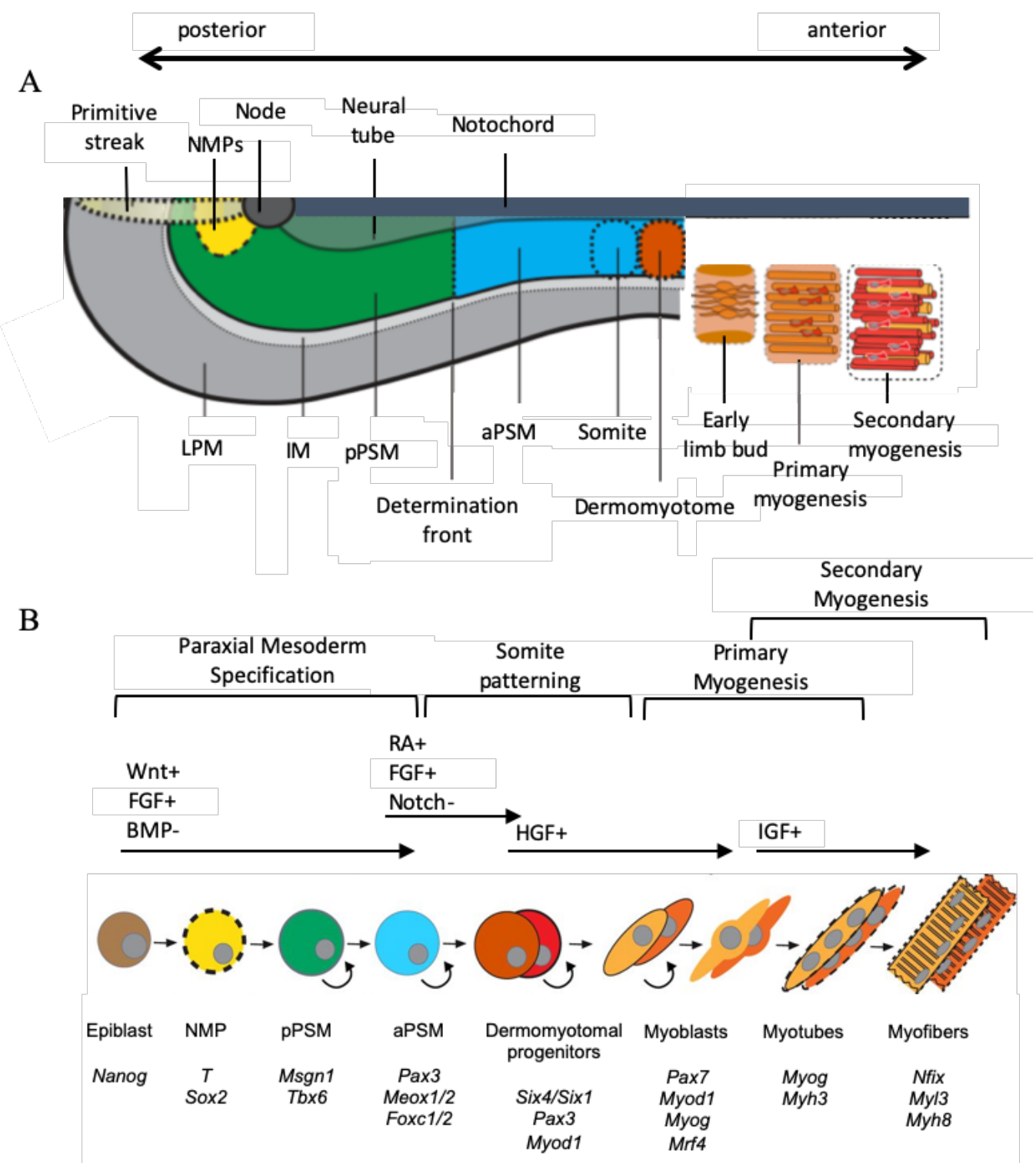

Figure 3. Skeletal myogenesis in vivo.

A, Schematic of dorsal view of spatial organization of mesoderm fate and limb skeletal myogenesis progression from posterior (left) to anterior (right) region of a developing amniote embryo. IM: intermediate mesoderm, LPM: lateralplate mesoderm, NMPs: neuromesodermal progenitors, pPSM: posterior presomitic mesoderm; aPSM: anterior presomitic mesoderm. B, Diagram of differentiation of paraxial mesoderm to skeletal muscle fibers of limb with color coded cell types according to the fate map in (A) are shown from left to right. Key signaling pathways identified during the in vivo differentiation of paraxial mesoderm and skeletal myogenesis as well as the corresponding marker genes are indicated. Foxc1: Forkhead-related transcription factor 1; Foxc2: Forkhead-related transcription factor 2; Myod1: Myogenic differentiation 1; Myog: Myogenin; Mrf4: Myogenic regulatory factor 4; Myh3: Myosine heavy chain 3; Myh7: Myosine heavy chain 7; Myh8: Myosine heavy chain 8; Myl3: Myosin light chain 3; Msgn1: Mesogenin 1; Meox1: Mesenchyme homeobox 1; Meox2: Mesenchyme homeobox 2; Nfix: Nuclear factor I X; NMPs: neuromesodermal progenitors, Pax3: Paired box gene 3; Pax7: Paired box gene 7; pPSM: posterior presomitic mesoderm; aPSM: anterior presomitic mesoderm. Six 1: Sine oculis-related homeobox homolog 1; Six4: Sine oculisrelated homeobox homolog 4; Sox2: SRY-box transcription factor 2; T: T-box transcription factor T; Tbx6: T-box transcription factor 6 (Image adapted with permission from Development, Company of Biologists, Chal and Pourquié 2017 and from Current Topics in Developmental Biology, Elsevier, Pourquié et al. 2018). 


\subsubsection{Somite patterning}

In the aPSM increasing level of retinoic acid (RA) and FGF signaling (Pourquié 2011; Yamaguchi et al. 1994) induce Notch signaling inhibition to establish the compartmentalization of the somite (Morimoto et al. 2005). The aPSM progressively condenses and coincidently somites exit as epithelial spheres from the dorsal-most region of aPSM. The cell population oriented towards the notochord will differentiate into the sclerotome via an epithelial-mesenchymal transition. The remaining epithelium forms the dermomyotome, which will later give rise to skeletal muscle and dermis (Tajbakhsh and Spörle 1998). Notch signaling inhibition in somites will promote dermomyotome formation (Mayeuf-Louchart et al. 2014).

The dermomyotome can anatomically be divided into dorsomedial (epaxial) and ventrolateral (hypaxial) compartments which will give rise to skeletal muscle of trunk and limb respectively (Figure 4) (Cinnamon et al. 2001). Dermomyotomal cells at the level of the limb skeletal myogenesis are characterized by expression of $\operatorname{Pax} 3$ and at the level of trunk skeletal myogenesis are characterized by expression of Myf5 (Bentzinger, Wang, and Rudnicki 2012; M Ying et al. 2011; Martins et al. 2009; Mayeuf-Louchart et al. 2014; Parker, Seale, and Rudnicki 2003).

\subsubsection{Skeletal myogenesis}

The myogenic program can be subdivided into primary/embryonic and secondary myogenesis. The epaxial dermomyotome which is specified by BMP inhibition will generate skeletal muscle of the myotome and later trunk and back muscles (Marcelle, Stark, and Bronner-Fraser 1997). During primary myogenesis the neural crest cells that migrate between the neural tube and somites by expressing the Notch ligand delta-like 1 (Dl11) will transiently activate Notch signaling in epaxial dermomyotome cells to express $M y f 5$ that rapidly will give rise to $M y o D$ expressing myoblasts (Borycki et al. 1999; Kablar et al. 1997; Rios et al. 2011; Rossi and Messina 2014).

The hypaxial dermomyotome will give rise to myogenic progenitors migrating to the early limb bud to develop limb and diaphragm muscles (Buckingham and Relaix 2007; Hirsinger et al. 2000). During primary myogenesis at the limb bud level, the transcription factors sine oculis-related homeobox 1 and 4 (Six 1 and Six4) guide the Pax 3 expressing progenitors from the dermomyotome towards the myogenic lineage (Figure 4) (Grifone et al. 2005; Laclef et al. 2003; Relaix et al. 2013). Pax3 controls c-met (tyrosine kinase receptor) expression (Epstein et al. 1996; Kassar- 
Duchossoy et al. 2005). c-met and its ligand, hepatocyte growth factor (HGF), regulate growth and migration of myogenic precursor cells derived from dermomyotome through the myogenic differentiation program by sequentially expressing the myogenic regulatory factors (MRFs) such as MyoD and Myogenin to give rise to primary myoblasts (F. Bladt et al. 1995).

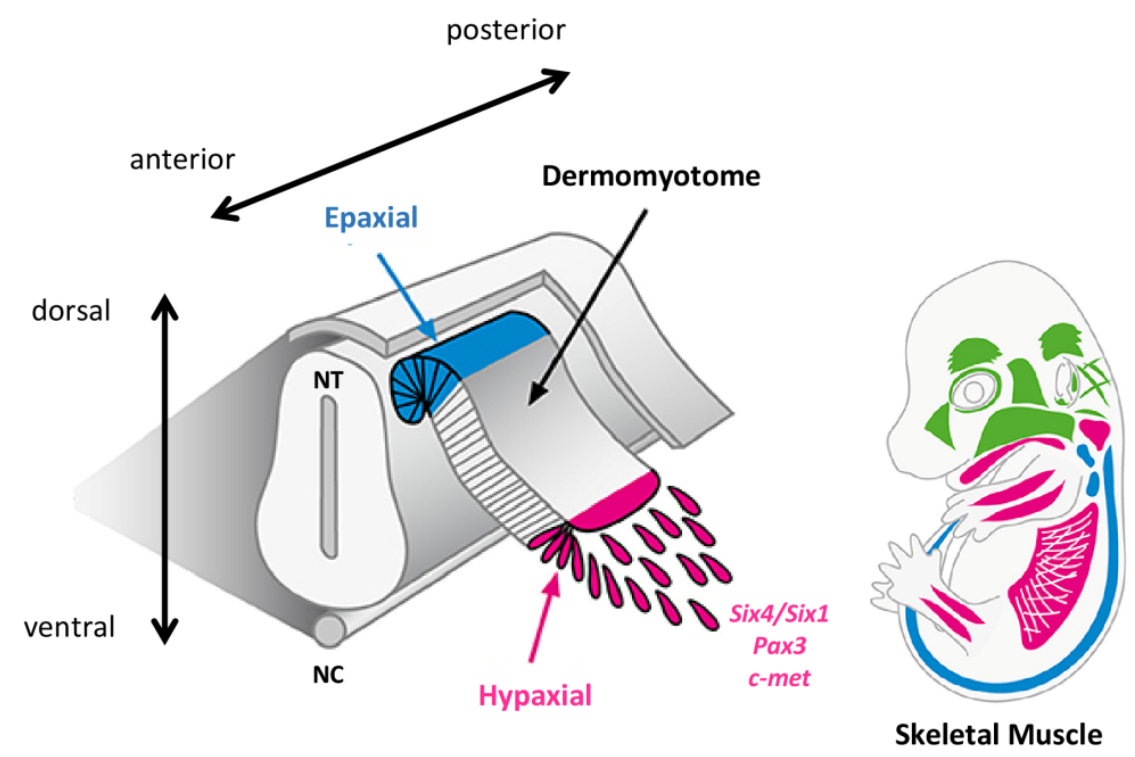

Figure 4. Origin of limb and trunk skeletal muscle.

Schematic of the dermomyotome that gives rise to epaxial (trunk and back) and hypaxial (limb and diaphragm) muscles. The hypaxial muscle progenitor cells are specified by Six4/Six 1, Pax3 and c-met that will migrate to the limb bud. NC: notochord; NT: neural tube (Image adapted with permission from frontiers in Cell and Developmental Biology, Nassari, Duprez, and Fournier-Thibault 2017).

Insulin-like growth factor (IGF) will promote myoblast fusion to form primary myotubes (van der Velden et al. 2006). These myotubes express specific proteins such as Myogenin and embryonic myosin heavy chain (Myh3).

In secondary or fetal/neonatal myogenesis a subset of the somitic Pax $3+$ myogenic progenitors express $\operatorname{Pax} 7$ and downregulate $\operatorname{Pax} 3$. These Pax $7+$ myogenic progenitors will proliferate and fuse to each other to form secondary myoblasts expressing transcription factor $N f i x$, or will fuse to the primary myotubes to give rise to the secondary or fetal fibers that expressing markers such as myosin light chain 3 (Myl3) and neonatal myosin heavy chain (Myh8) (Biressi, Molinaro, and 
Cossu 2007; Buckingham and Relaix 2007; Messina et al. 2010; White et al. 2010). The expression of the transcription factor Nfix is induced by Pax7+ myogenic progenitors in fetal muscle, activating the transcription of fetal specific genes such as muscle creatin kinase $(\mathrm{Ckm})$. Thus, $\mathrm{Nfix}$ has been proposed to control the switch from embryonic to fetal myogenesis (Messina et al. 2010). Six factors (Sixl and Six4) and the transcriptional repressor Sox6 are also controlling the gene program of fast muscle fibers at the stage of the switch from embryonic to fetal myogenesis (Hagiwara, Yeh, and Liu 2007; Niro et al. 2010).

\subsubsection{Postnatal muscle growth}

While a detailed characterization of the dynamics of postnatal skeletal muscle growth and maturation is lacking (Gattazzo et al. 2020), the current knowledge postulates that during postnatal stage the number of muscle fibers remains constant, while the size of each muscle fiber starts to increase by hypertrophy through the accretion of muscle protein within growing fibers and an increase in the number of myonuclei through fusion of differentiating PAX7+ satellite cells to the growing muscle fiber(Goldspink 1970; Montgomery 1962; White et al. 2010).

During postnatal growth, muscle fibers will lose polyneuronal innervation and different stimuli such as mechanical loading and thyroid hormone level will induce the size-independent maturation process in skeletal muscle including the isoform transformation of myosin heavy chain (MYH) in the muscle fiber. Postnatally skeletal muscle expression of immature MYH like embryonic myosin heavy chain (Myh3) and neonatal myosin heavy chain (Myh8) decreases while the expression of adult myosin heavy chain isoforms increases (Gambke et al. 1983; Schiaffino et al. 1988, 2015). In principle, adult myosin heavy chain polymorphism gives rise to four major types of fibers with different contractile properties distributed in varying levels in limb, trunk, and head muscle tailored to the specific motor performance of the respective muscle. Type I fibers express myosin heavy chain 7 (Myh7), type IIa fibers express myosin heavy chain 2 (Myh2), type IIx fibers express myosin heavy chain 1 (Myhl) and type IIb fibers express myosin heavy chain 4 (Myh4). ATPase activity of myosin isoforms categorizes the type I fibers as slow-twitch fibers with sustained tension, whereas type II fibers are fast-twitch fibers performing short burst activities with rapid decline in tension following repetitive stimulation. (Schiaffino and Reggiani 2011). 
Neuronal activity and molecular make-up of the neuromuscular junctions (NMJ), a synapse between a motor neuron and nicotinic acetylcholine receptors (nAChRs) in the postsynaptic membrane of the muscle fiber, are determinants of the skeletal muscle fiber development. Nerveinduced muscle contraction is required for correct myofibrillar growth in developing muscle fibers and for the selective expression of AChR subunits at the NMJ by synapse-associated nuclei. All embryonic muscles express the gamma subunit AChRs exclusively while the majority of adult muscle fibers express epsilon subunit and have no detectable gamma subunit (Afshar Bakooshli et al. 2019; Fambrough 1979; Missias et al. 1996).

\subsection{Contractile function of skeletal muscle}

Skeletal muscle contraction begins with propagation of action potentials from the motor neuron to the muscle cell through the neuromuscular junction, a cholinergic synapse between a motoneuron and a muscle fiber. Following the release of acetylcholine (ACh) into the synaptic cleft, ACh will bind to the nicotinic receptors (nAChR), ligand-gated ion channels located on the sarcolemma. Binding of Ach will lead to influx of sodium ions which depolarizes the membrane and triggers the excitation-contraction coupling via release of calcium from the SR to generate tension. One action potential causes a single muscle twitch and multiple action potentials at maximal stimulation frequencies result in summation of twitches leading to tetanic tension development (Figure 5A).

Tension development reflects the $\mathrm{Ca}^{2+}$ dependent actin-myosin cross bridge formation in the sarcomere (Racca et al. 2013). Both sarcomeric arrangements and actin-myosin cross bridge detachment define the tension relaxation. The rate of tension development and relaxation is significantly slower in human fetal myofibrils than adult ones (Figure 5B) (Poggesi, Tesi, and Stehle 2005). Twitch tension of skeletal muscle significantly increases with maturation which is postulated to be mainly caused by increased sarcomere alignment and myofibril density. Human fetal myofibrils produce significantly less force than adult ones (Racca et al. 2013). 

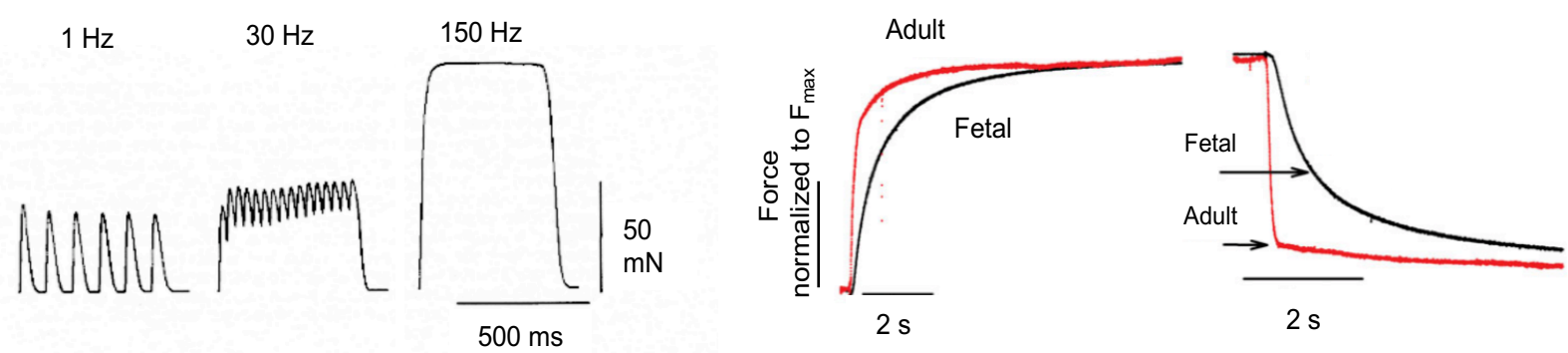

Figure 5. Force trace patterns of mammalian muscle fibers.

A, Representative force traces of single twitches at $1 \mathrm{~Hz}$, unfused tetanus at $30 \mathrm{~Hz}$ and fused tetanus at $150 \mathrm{~Hz}$ of adult rat fast muscle motor units (Image adapted with permission from journal of Physiology and Pharmacology, Celichowski 2000). B, Normalized tetanic force traces presenting the slower activation and relaxation kinetics of human fetal myofibril (black line) in comparison with the adult counterpart (red line) (Image adapted with permission from Journal of Physiology Racca et al. 2013).

\subsection{Skeletal muscle regeneration}

\subsubsection{Origin of satellite cells}

The self-renewing stem cell of skeletal muscle is called satellite cell. Pax7+ myogenic progenitors originating from somites are the source of adult satellite cells (Gros et al. 2005; Seale et al. 2000). During late neonatal stage the pool of satellite cell progenitors represents up to $30 \%$ of the mononucleated cells and while they are actively dividing some of these Pax7+ cells exit the cell cycle and localize to the muscle fiber underneath the basal lamina to establish the quiescent satellite cell niche (Figure 6A) (Hellmuth and Allbrook 1971; Mauro 1961).

By adulthood the proportion of satellite cell progenitors reduces to a small pool (5-6\%) of mitotically quiescent satellite cells (Allbrook, Han, and Hellmuth 1971; Schultz 1974). In adult skeletal muscle, satellite cells express a set of markers including the paired domain transcription factors Pax7 (Figure 6B,C) and Pax3 (Buckingham et al. 2003; Seale et al. 2000), myogenic regulatory factor Myf5 (Cornelison and Wold 1997), homeobox transcription factor Barx2 (Meech et al. 2012), tyrosine kinase receptor c-Met (Allen et al. 1995) and Caveolin-1 (Volonte, Liu, and Galbiati 2005). Specifically, Pax7 is the canonical marker for quiescent and proliferating satellite cells (Seale et al. 2000). 

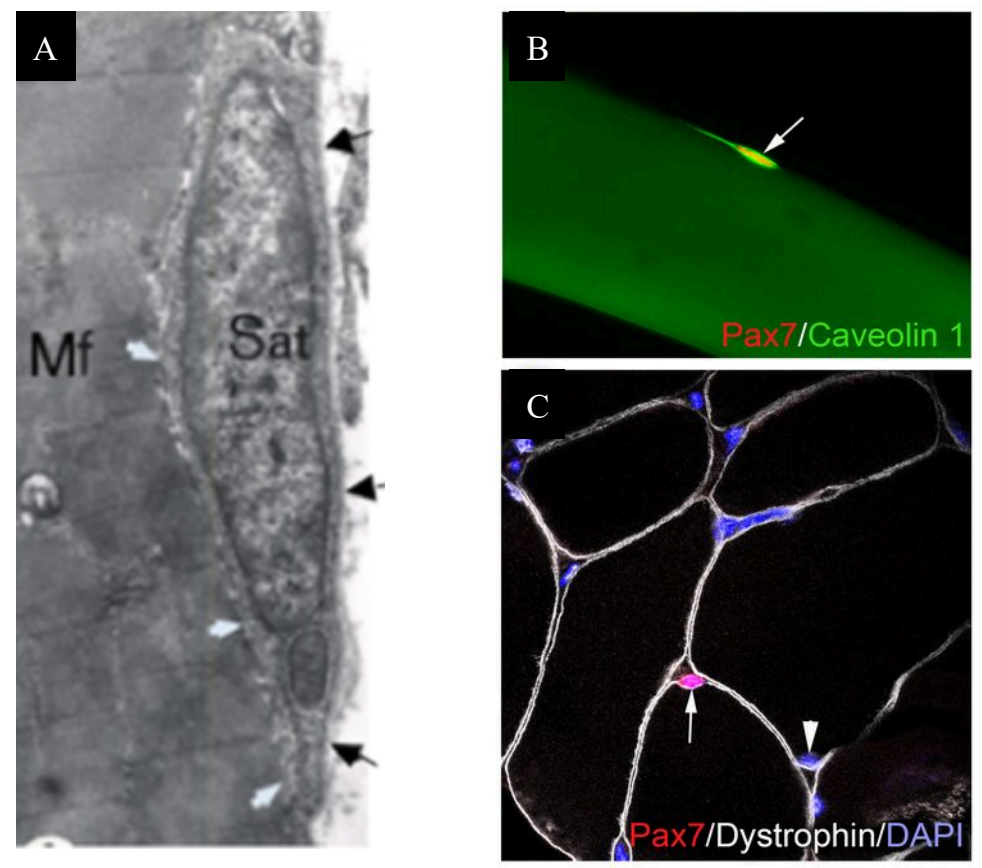

Figure 6. Satellite cell niche of skeletal muscle.

A, Transmission electron microscopy image of satellite cell (white arrow) sitting under the basal lamina (Black arrow) of muscle fiber. Mf: Muscle fiber; Sat: Satellite cell (Image adapted with permission from Journal of Histochemistry and Cytochemistry, Yablonka-Reuveni 2011). B, Immunostaining for Pax7 (red-nuclear) and Caveolin-1 (green) to reveal a satellite cell (indicated by an arrow) resting in its niche adjacent to a muscle fiber isolated from an adult mouse (Image adapted with permission from Development, The Company of Biologists, Relaix and Zammit 2012). C, Immunostaining of a cross section of an adult mouse muscle for Pax7+ satellite cell (red) indicated by arrow, Dystrophin (white) to delineate the sarcolemma of the muscle fiber and DAPI (blue). The arrowhead highlights a myonucleus (Image adapted with permission from Development, The Company of Biologists, Relaix and Zammit 2012).

\subsubsection{Sequential stages of skeletal muscle regeneration}

Upon muscle injuries caused by resistance training, exposure to myotoxins or trauma, the resulting muscle fiber loss will be regenerated (Luz, Marques, and Santo Neto 2002) by satellite cells breaking quiescence, proliferating, and differentiating into satellite cell-derived myoblasts. These myoblasts then fuse and give rise to immature myotubes (with central myonuclei) and finally muscle fibers (Bischoff 1975; Sambasivan et al. 2011).

Specific signaling pathways such as Notch and HGF signaling are implicated in activation of satellite cells. Notch signaling is on one hand crucial for maintaining the satellite cell quiescence (Mourikis et al. 2012) and on the other hand it is essential for activation and proliferation of satellite cells. Following injury, both satellite cells and myofibers upregulate expression of Notch1 
resulting in activation of the satellite cell, which in turn will proliferate (Conboy and Rando 2002). Inhibition of Notch signaling has been shown to block regeneration in rat engineered skeletal muscle (Tiburcy et al. 2019). In the early phase of muscle regeneration, HGF is sequestered by heparan sulfate proteoglycans within the basal lamina. HGF will bind to cell surface receptor cMet on both quiescent and activated satellite cells to stimulate proliferation (Cornelison and Wold 1997; Miller et al. 2000; Tatsumi and Allen 2004). Proliferative satellite cells will rapidly differentiate to MyoD expressing myoblasts and begin to fuse to damaged myofibers or fuse to each other to form new myofibers (Yin, Price, and Rudnicki 2013).

\subsection{Human in vitro models of skeletal muscle}

\subsubsection{Directed skeletal myocyte differentiation of human pluripotent stem cells}

To enhance the translational impact of experimental models for muscle disease, relevant human in vitro models are a promising tool. Although primary human myoblasts are available, typically limited numbers can be obtained from biopsies and genetic modification to interrogate genotypephenotype relations is nearly impossible in primary cells. Recently, encouraging progress has been made in differentiating human pluripotent stem cells (hPSCs) into a myogenic fate in two dimensional culture without the need of genetic modification such as overexpression of Pax7 or MyoD (Chal et al. 2016; Choi et al. 2016).

The protocol provided by Chal et al. 2016 starts with dual modulation of Wnt and BMP signaling pathways, followed by FGF signaling activation to generate induced paraxial mesoderm cells (iPAMs). In the next steps iPAMs were exposed to growth factors HGF and IGF1 to be differentiated into trunk skeletal myogenic cells within 30 days. By this approach they obtained a myogenic differentiated culture with 22\% Myogenin+ nuclei and 23\% PAX7+ nuclei (Chal et al. 2016; Pourquié et al. 2018). In the protocol provided by Choi et al. only Wnt signaling is activated to obtain presomitic progenitors. Next, to increase the speed and efficiency of myogenic specification, Notch signaling is blocked. The reported yield of myogenic cells at day 30 is $61 \%$ Myogenin and 63\% Myosin (labelled by MF20 antibody) expressing cells (Choi et al. 2016). 
While there is significant progress in skeletal muscle differentiation from human PSCs the limitations of 2D monolayer cultures in supporting functional properties of mature muscle fibers are inevitable (Afshar Bakooshli et al. 2019; Chal et al. 2016). Therefore, in vitro generation of 3 dimensional (3D) models of human skeletal muscle with enhanced maturation is important to offer more desirable physiological platforms for experimental and therapeutic testing. Tissue engineering may address some of these limitations.

\subsubsection{Tissue engineering of skeletal muscle}

Tissue engineering is a promising tool to add a third dimension to monolayer culture models. Tissue engineering of skeletal muscle from rodent and human primary cells has been pioneered more than 30 years ago (Vandenburgh, Karlisch, and Farr 1988). Work from our own group demonstrated that engineered skeletal muscle from rat cells faithfully recapitulates skeletal muscle physiology including the presence of a functional satellite cell niche that regenerates the muscle in vitro (Tiburcy et al. 2019).

Only recently, three models of tissue engineered skeletal muscle from hPSCs have been reported (Figure 7) by using either forced expression of Pax7 (Rao et al. 2018; Xu et al. 2019) or directed multi-lineage differentiation (Maffioletti et al. 2018). 
A
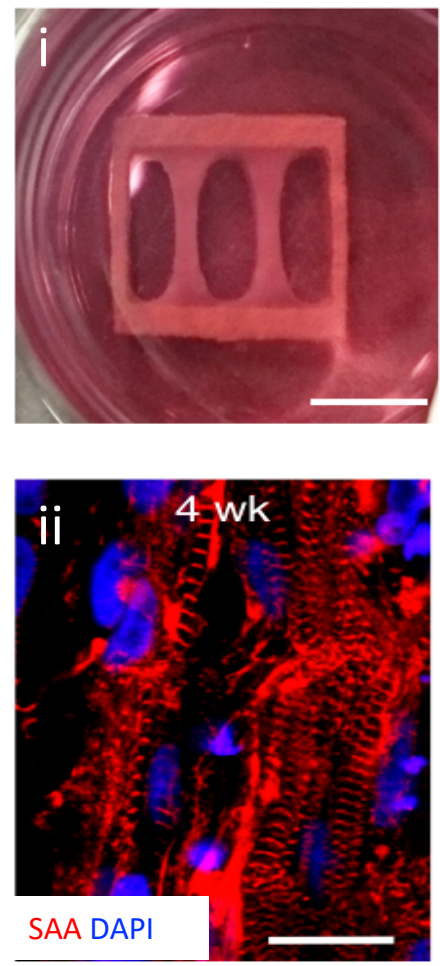

$\mathrm{B}$
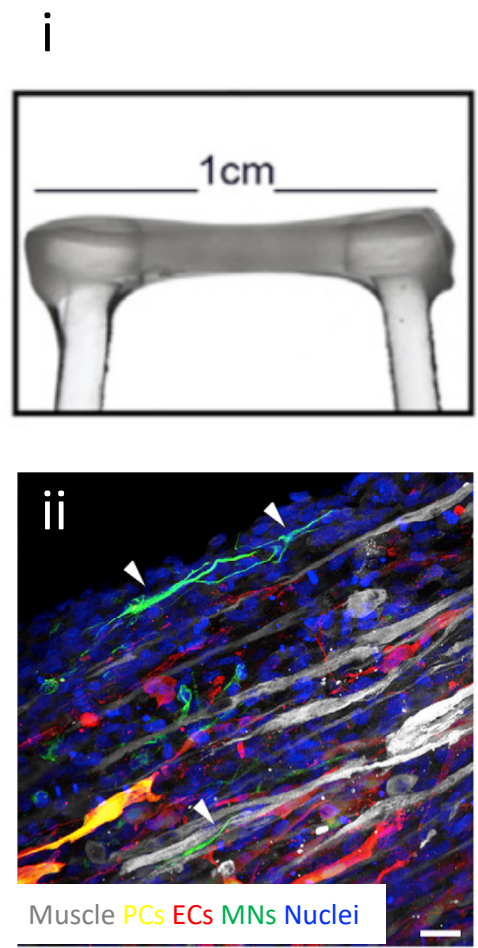

C
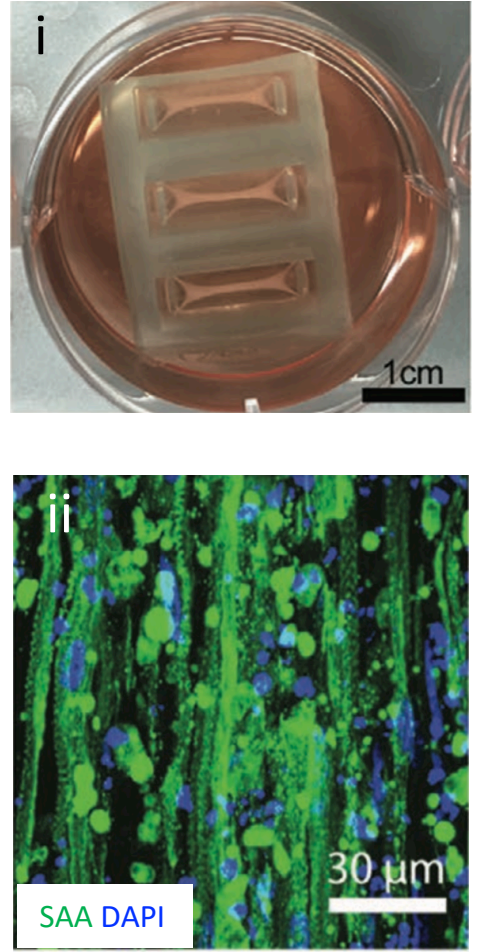

Figure 7. Tissue engineering models of skeletal muscle from hPSCs.

A, (i) Human skeletal muscle bundle generated from Pax7 induced myogenic progenitor cells, anchored within a nylon frame. (ii) Immunostaining of cross striated myotube for sarcomeric $\alpha$-actinin (SAA, red) and myonuclei (DAPI, blue) in a 4 wk muscle bundle. Scale bar: $10 \mu \mathrm{m}$ (Image adapted with permission from Nature Communications, Rao et al. 2018). B, (i) Side view of the multilineage 3D artificial skeletal muscle model from iPSC. (ii) Immunofluorescent image of multilineage 3D artificial muscle derived from WT human iPSCs containing isogenic myofibers (Muscle, gray), vascular cells (ECs, red and PCs, yellow), and motor neurons indicated by arrowheads (SMI32, green). ECs: endothelial cells; PCs: pericytes. Scale bar: $10 \mu \mathrm{m}$ (Image adapted with permission from Cell Reports, Maffioletti et al. 2018). C, (i) Three representative muscle constructs generated from PAX7-induced hESC-derived myogenic progenitors. (ii) Immunostaining of aligned myotubes and striated sarcomere structures indicated by sarcomeric $\alpha$ actinin (SAA, green) (Image adapted with permission from Advanced Biosystems, Xu et al. 2019).

An ideal tissue engineered skeletal muscle should display all characteristic morphological (e.g. formation of adult muscle fiber syncytium with a proper niche for satellite cells) and functional (e.g. tetanic contractions upon high frequency stimulation) properties of bona fide skeletal muscle to be eligible for downstream applications in regenerative medicine and disease modeling (Madden et al. 2015; Tiburcy et al. 2019). Advanced tissue engineering approaches to provide a 3D culture environment containing not only differentiated human PSCs-derived skeletal myocytes, but also 
supportive non-myocyte populations and exhibiting robust skeletal muscle function are still in need.

\subsubsection{Duchenne muscular dystrophy in in vitro models of human skeletal muscle}

Duchenne muscular dystrophy (DMD) is a fatal, $\mathrm{X}$-linked recessive disorder caused by mutations in $D M D$ gene encoding Dystrophin protein. DMD patients face a progressive muscle weakness with muscle fibrosis and futile regeneration at early age. Weakness of the diaphragm and cardiomyopathy become clinically apparent with advanced disease and cause life threatening breathing difficulties and cardiac failure.

Dystrophin and its associated protein complex (dystroglycans, sarcoglycans, sarcospan, $\alpha$ dystrobrevins, syntrophins, syncoilin, nNOS, and caveolin-3 (Ehmsen, Poon, and Davies 2002)) anchors the sarcolemma to the $\mathrm{Z}$ disk of the sarcomere as force generating unit in striated muscle (Lapidos, Kakkar, and McNally 2004). In skeletal muscle, lack of dystrophin renders muscle fibers more susceptible to membrane damage during increased mechanical stress (Petrof et al. 1993). While rodent (Amoasii et al. 2017) and large animal models (Amoasii et al. 2018; Moretti et al. 2020) of DMD have been established, there is no doubt that the clinical and physiological aspects of human DMD mutations cannot fully be recapitulated in animal models (van der Worp et al. 2010). In vitro 3D models of human skeletal muscle carrying patient-specific mutations are at least complementary to animal models, providing more comprehensive approaches to genotypephenotype relations of human dystrophic skeletal muscle disease. As an example, it is demonstrated that CRISPR/Cas9-mediated "myoediting" of DMD mutations restores dystrophin expression and the corresponding force of contraction in a 3D model of engineered heart muscle (EHM) from patient specific iPSC-derived cardiomyocytes (Long et al. 2018). In another study Maffioletti et al, presented that a 3D skeletal muscle construct can stimulate differentiation of dystrophic human PSCs, modeling severe and incurable forms of muscular dystrophy (Maffioletti et al. 2018). 


\subsection{Aim and hypotheses of the study}

Considering the significance of human skeletal muscle disease we aimed to generate engineered skeletal muscle from human PSC to model skeletal muscle disease in vitro.

To achieve this goal, we investigated the following specific hypotheses:

1) A robust protocol for directed skeletal muscle differentiation from human PSCs can be established by recapitulating embryonic developmental cues.

2) Human engineered skeletal muscle with physiological function can be generated in vitro.

3) Human engineered skeletal muscle regenerates after injury in vitro.

4) Human engineered skeletal muscle recapitulates the phenotype of patients with Duchenne Muscular Dystrophy. 


\section{Materials and Methods}

\subsection{Human cell culture techniques}

\subsubsection{Human pluripotent stem cell lines}

The following human pluripotent stem cell (hPSC) lines were utilized in this project.

Table 1. List of human PSC lines

\begin{tabular}{|c|c|c|c|}
\hline Line & Description & Source & Identifier \\
\hline HES2 & Human embryonic stem cell line & WiCell & $\begin{array}{l}\text { (Reubinoff et al. } \\
\text { 2000) }\end{array}$ \\
\hline iPSC 1 & Human wild type line TC-1133 (WT1) & RUDCR & $\begin{array}{l}\text { (Baghbaderani } \\
\text { et al. 2016) }\end{array}$ \\
\hline iPSC 2 & Human wild type line(WT2) & $\begin{array}{l}\text { D. Garry, University of } \\
\text { Minnesota }\end{array}$ & $\begin{array}{l}\text { (Long et al. } \\
2018)\end{array}$ \\
\hline iPSC 3 & $\begin{array}{l}\text { Human DMD line (DMD del; deletion } \\
\text { of exon } 48-50\end{array}$ & $\begin{array}{l}\text { E. Olson, University of } \\
\text { Texas, Southwestern }\end{array}$ & $\begin{array}{l}\text { (Long et al. } \\
2018)\end{array}$ \\
\hline iPSC 4 & $\begin{array}{l}\text { Human DMD iPSC line (DMD del cor; } \\
\text { corrected by myoediting }\end{array}$ & $\begin{array}{l}\text { E. Olson, University of } \\
\text { Texas, Southwestern }\end{array}$ & $\begin{array}{lll}\text { (Long et al. } \\
\text { 2018) }\end{array}$ \\
\hline iPSC 5 & $\begin{array}{l}\text { Human DMD iPSC line (DMD pEx; } \\
\text { pseudoexon 47A) }\end{array}$ & $\begin{array}{l}\text { E. Olson, University of } \\
\text { Texas, Southwestern }\end{array}$ & $\begin{array}{l}\text { (Long et al. } \\
2018 \text { ) }\end{array}$ \\
\hline iPSC 6 & $\begin{array}{l}\text { Human DMD iPSC line (DMD pEx cor; } \\
\text { corrected by myoediting) }\end{array}$ & $\begin{array}{l}\text { E. Olson, University of } \\
\text { Texas, Southwestern }\end{array}$ & $\begin{array}{lll}\text { (Long et al. } \\
\text { 2018) }\end{array}$ \\
\hline
\end{tabular}

All lines were routinely tested for pluripotency and confirmed to be free of mycoplasma (Lonza Mycoalert $^{\mathrm{TM}}$ kit). 


\subsubsection{Ethics statement}

The use of HES2 line was approved by the Robert-Koch-Institute (Nr. 3.04.02/0160).

\subsubsection{Human pluripotent stem cell culture}

Human PSC lines were maintained on 1:120 Matrigel $^{\mathrm{TM}}$ (BD) in phosphate-buffered saline (Thermo Fisher Scientific)-coated plates and cultured in StemMACS iPS-Brew XF (Miltenyi Biotec) at $37{ }^{\circ} \mathrm{C}$ and $5 \% \mathrm{CO}_{2}$. Medium was changed every day and when the culture reached a confluency of 80-90\%, it was rinsed once with PBS (Thermo Fisher Scientific) and incubated in Versene solution (Thermo Fisher Scientific) for 3-5 $\mathrm{min}$ at room temperature. Versene was carefully aspirated and cells were washed gently with StemMACS iPS-Brew XF (Miltenyi Biotec) supplemented with $5 \mu \mathrm{M}$ Y27632 (Stemgent). Cells were counted (CASY cell counter) and passaged every 3 to 4 days at a 1:6 to $1: 8$ ratio by plating $1 \times 10^{6}$ cells into a new T75 flask.

\subsubsection{Skeletal muscle differentiation media and reagents}

To perform the skeletal muscle differentiation, the following media and reagents were prepared (Table 2). A complete list of media and reagents can be found in the Appendix, Table A1.

Note: All media were warmed up to $20-24^{\circ} \mathrm{C}$ prior to use.

Table 2. List of media and reagents

\begin{tabular}{|l|l|}
\hline CHIR 99021 stock solution & $\begin{array}{l}\text { Reconstitute } \mathrm{CHIR}_{99021} \text { in DMSO to obtain a } 10 \mathrm{mM} \text { stock } \\
\text { solution. Aliquot and store at }-20^{\circ} \mathrm{C} \text { for up to one year. Once } \\
\text { thawed, keep at } 4^{\circ} \mathrm{C} \text { for up to one week. }\end{array}$ \\
\hline $\mathrm{LDN}_{193189}$ stock solution & $\begin{array}{l}\text { Reconstitute } \mathrm{LDN}_{193189} \text { in DMSO to obtain a } 10 \mathrm{mM} \text { stock } \\
\text { solution. Aliquot and store at }-20^{\circ} \mathrm{C} \text { for up to one year. Once } \\
\text { thawed, keep at } 4^{\circ} \mathrm{C} \text { for up to one week. }\end{array}$ \\
\hline
\end{tabular}




\begin{tabular}{|c|c|}
\hline DAPT stock solution & $\begin{array}{l}\text { Reconstitute DAPT in DMSO to obtain a } 20 \mathrm{mM} \text { stock solution. } \\
\text { Aliquot and store at }-20^{\circ} \mathrm{C} \text { for up to one year. Once thawed, keep } \\
\text { at } 4^{\circ} \mathrm{C} \text { for up to one week. }\end{array}$ \\
\hline FGF-2 stock solution & $\begin{array}{l}\text { Dissolve FGF-2 in PBS containing } 0.1 \% \text { recombinant albumin to } \\
\text { obtain a } 10 \mu \mathrm{g} / \mathrm{ml} \text { stock solution. Aliquot and store at }-20^{\circ} \mathrm{C} \text { for up } \\
\text { to one year. } \\
\text { Once thawed, keep at } 4{ }^{\circ} \mathrm{C} \text { for up to one week. }\end{array}$ \\
\hline HGF stock solution & $\begin{array}{l}\text { Dissolve HGF in PBS containing } 0.1 \% \text { recombinant albumin to } \\
\text { obtain a } 10 \mu \mathrm{g} / \mathrm{ml} \text { stock solution. Aliquot and store at }-20^{\circ} \mathrm{C} \text { for up } \\
\text { to one year. } \\
\text { Once thawed, keep at } 4{ }^{\circ} \mathrm{C} \text { for up to one week. }\end{array}$ \\
\hline N2 medium & 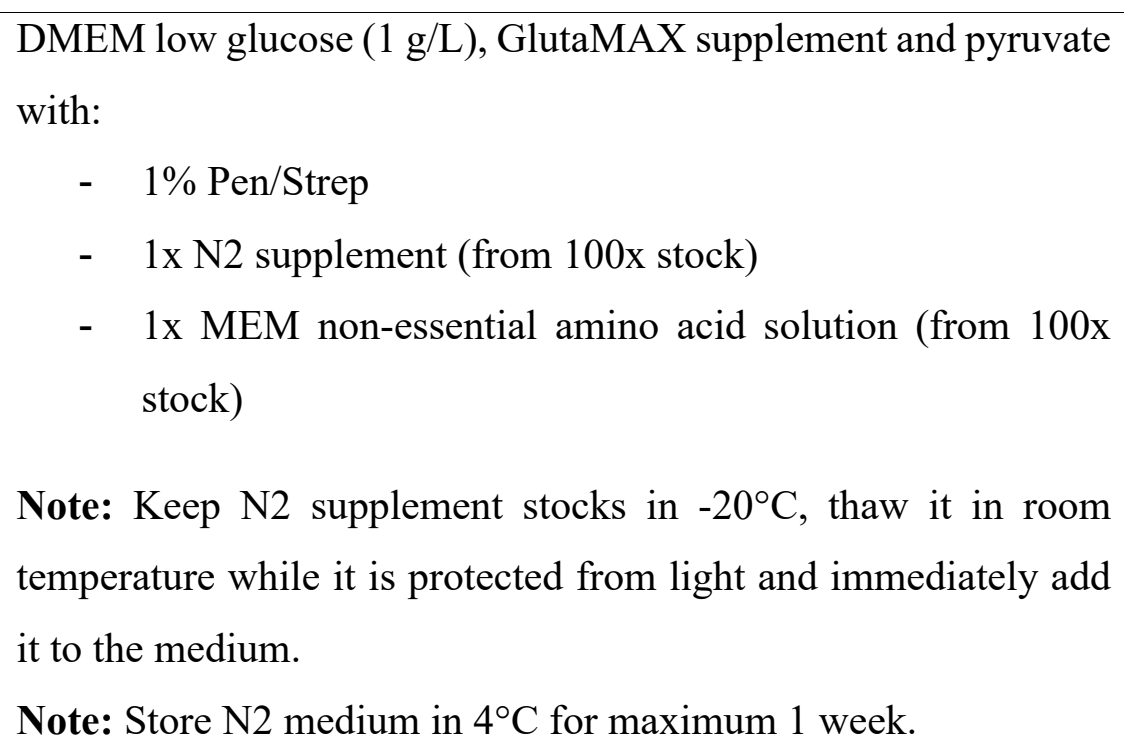 \\
\hline $\begin{array}{l}\text { N2-CLF medium } \\
\text { (day } 0,1,2 \text { and } 3 \text { ) }\end{array}$ & $\begin{array}{l}\text { N2 medium plus: } \\
\text { - } 10 \mu \mathrm{mol} / \mathrm{L} \text { CHIR99021 (C), must be added freshly before } \\
\text { use. } \\
\text { - } \quad 0.5 \mu \mathrm{mol} / \mathrm{L} \mathrm{LDN}_{193189}(\mathrm{~L}), \text { must be added freshly before } \\
\quad \text { use. } \\
\text { - } 10 \mathrm{ng} / \mathrm{ml} \mathrm{FGF-2} \mathrm{(F),} \mathrm{must} \mathrm{be} \mathrm{added} \mathrm{freshly} \mathrm{before} \mathrm{use.}\end{array}$ \\
\hline
\end{tabular}




\begin{tabular}{|c|c|}
\hline $\begin{array}{l}\text { N2-FD medium } \\
\text { (day } 4 \text { and 5) }\end{array}$ & $\begin{array}{l}\text { N2 medium plus: } \\
\begin{aligned}- & 20 \mathrm{ng} / \mathrm{ml} \text { FGF-2 (F), must be added freshly before use. } \\
- & 10 \mu \mathrm{mol} / \mathrm{L} \text { DAPT (D), must be added freshly before use. }\end{aligned}\end{array}$ \\
\hline $\begin{array}{l}\text { N2-FDH medium } \\
\text { (day } 6 \text { and 7) }\end{array}$ & $\begin{array}{l}\text { N2 medium plus: } \\
\begin{aligned}- & 20 \mathrm{ng} / \mathrm{ml} \text { FGF-2 (F), must be added freshly before use. } \\
- & 10 \mu \mathrm{mol} / \mathrm{L} \text { DAPT (D), must be added freshly before use. } \\
- & 10 \mathrm{ng} / \mathrm{ml} \text { HGF (H), must be added freshly before use. }\end{aligned}\end{array}$ \\
\hline $\begin{array}{l}\text { N2-DHK medium } \\
\text { (day } 8,9,10 \text { and } 11 \text { ) }\end{array}$ & 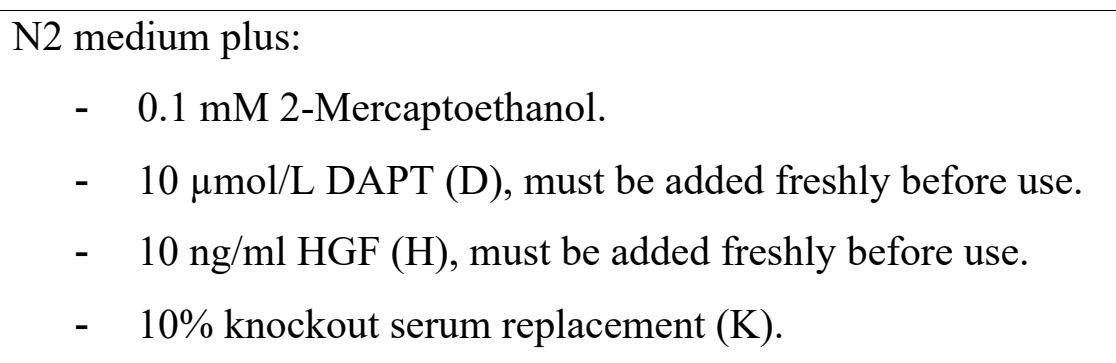 \\
\hline Expansion medium & 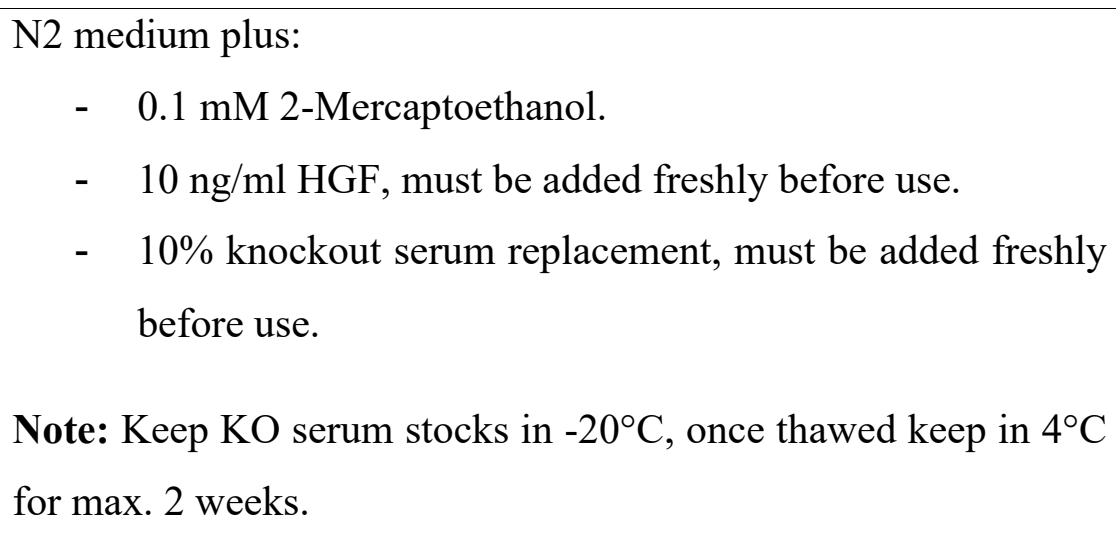 \\
\hline Maturation medium & 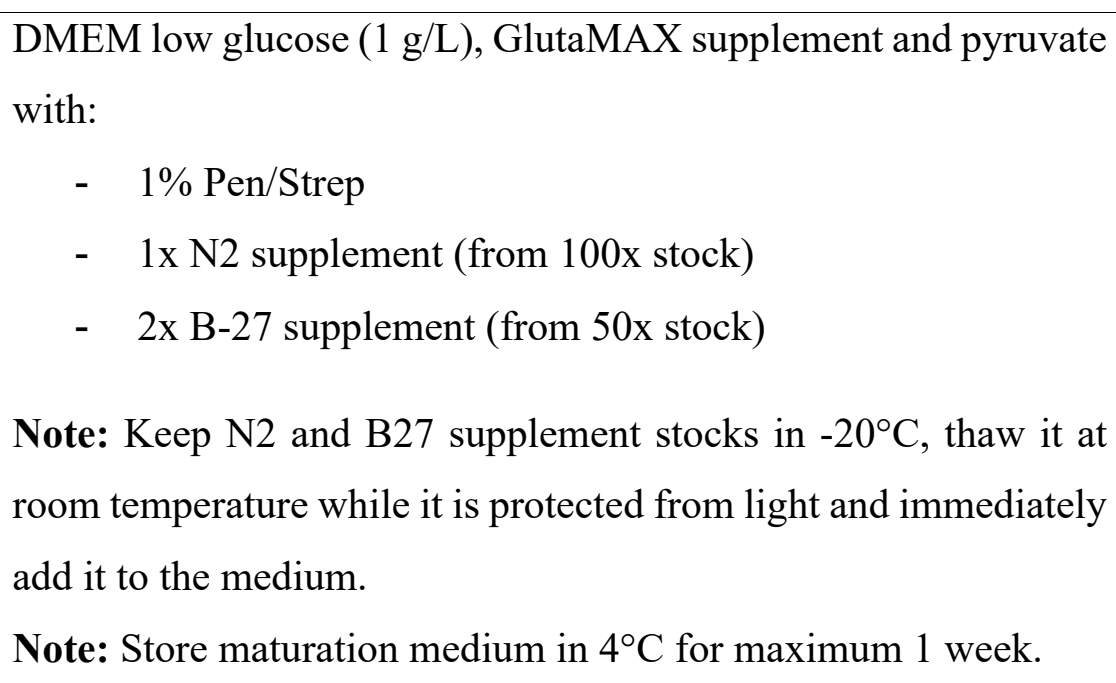 \\
\hline
\end{tabular}




\begin{tabular}{|c|c|}
\hline $\begin{array}{l}\text { Maturation medium plus } \\
\text { T3 and Creatine } \\
\text { (MM-TC) }\end{array}$ & $\begin{array}{l}\text { Maturation medium plus: } \\
\begin{array}{l}\text { - } \quad 1 \mathrm{mM} \text { Creatine monohydrate } \\
\text { - } \quad 0.1 \mu \mathrm{mol} / \mathrm{L} \text { Triiodo-L-thyronine (T3) }\end{array}\end{array}$ \\
\hline Y27632 stock solution & $\begin{array}{l}\text { Reconstitute Y27632 in DMSO to obtain a } 10 \mathrm{mM} \text { stock solution. } \\
\text { Aliquot and store at }-20^{\circ} \mathrm{C} \text { for up to one year. Once thawed, keep } \\
\text { at } 4^{\circ} \mathrm{C} \text { for up to one week. }\end{array}$ \\
\hline Matrigel & $\begin{array}{l}\text { To dilute Matrigel: } \\
\text { a. Thaw Matrigel ( } 7-10 \mathrm{mg} / \mathrm{ml} \text { ) on ice at } 4^{\circ} \mathrm{C} \text { for } 16-24 \mathrm{~h} \text {. } \\
\text { b. Prepare } 250 \mu l \text { aliquots into } 50 \mathrm{ml} \text { falcon tubes using ice-cold } \\
\text { pipette tips and tubes, and directly freeze. Store aliquots at - } \\
20^{\circ} \mathrm{C} \text { for up to one year. } \\
\text { To coat cell culture plates (e.g. T75 flask): } \\
\text { c. Resuspend a frozen Matrigel aliquot in } 29.75 \mathrm{ml} \text { cold PBS to } \\
\text { obtain the working dilution of } 1: 120 \text {. } \\
\text { d. Add } 6 \text { ml of diluted Matrigel per } \mathrm{T} 75 \text { flask and ensure that } \\
\text { the entire surface is covered. } \\
\text { e. Incubate at } 37^{\circ} \mathrm{C} \text { for } 60 \text { min before plating the cells. } \\
\text { Note: Coated plates can be stored in } 4^{\circ} \mathrm{C} \text { for up to } 2 \text { weeks, but } \\
\text { should not be used if the Matrigel has dried up. }\end{array}$ \\
\hline
\end{tabular}

\subsubsection{Directed differentiation of human PSCs into skeletal myocytes}

Human PSCs were plated at $1.7 \times 10^{4}$ cells $/ \mathrm{cm}^{2}$ on 1:120 Matrigel $^{\mathrm{TM}}$ (BD) in phosphate-buffered saline (Thermo Fisher Scientific) -coated plates and cultured in StemMACS iPS-Brew XF (Miltenyi Biotec) with $5 \mu \mathrm{M}$ of Y27632 (Stemgent). After 24h when the culture reached a confluency of 30\% (day 0), iPS-Brew XF was replaced with daily refreshed N2-CLF medium (Table 2) for 4 days. Differentiating cultures were highly delicate at this stage, therefore medium changes were done slowly to avoid cell detachment. At day 4, the medium was changed every day 
to N2-FD medium (Table 2) until day 6. For day 6 and 7 the medium was replaced daily with N2FDH medium (Table 2). The medium was switched to N2-DHK medium (Table 2) on day 8, 9, 10 and 11. From day 12 to 22, myogenic cells were cultured in expansion medium (Table 2) which was refreshed every second day. Day 22 differentiated cultures were dissociated for downstream applications including culture expansion, myogenic maturation, freezing and generation of human engineered skeletal muscle.

\subsubsection{Enzymatic dissociation of human PSC-derived skeletal myocytes}

Day 22 differentiated cultures were rinsed once with PBS (Thermo Fisher Scientific). TrypLE (Thermo Fisher Scientific) was added to the cells and incubated for 5 to 7 minutes at $37^{\circ} \mathrm{C}$ and $5 \% \mathrm{CO}_{2}$. TrypLE digestion was stopped using expansion medium with $5 \mu \mathrm{M}$ Y27632 (Stemgent). Cell suspension was triturated very gently with a $10-\mathrm{ml}$ serological pipette to break up the cell clumps and centrifuged at $100 \mathrm{xg}, 10$ minutes at $20-24^{\circ} \mathrm{C}$. Supernatant was removed and the pellet was resuspended very gently in expansion medium with $5 \mu \mathrm{M}$ Y27632 (Stemgent). Cells were replated on 1:120 Matrigel $^{\mathrm{TM}}$ (BD) in phosphate-buffered saline (Thermo Fisher Scientific)-coated plates at a density of $60-70,000$ cells $/ \mathrm{cm}^{2}$ in expansion medium with $5 \mu \mathrm{M}$ Y27632 (Stemgent). From the next day, the expansion medium was refreshed every other day for one week. To further differentiate the cells to myotubes in monolayer culture expansion medium was replaced with maturation medium (Table 2) for another 4 weeks. Where indicated $0.1 \mu \mathrm{mol} / \mathrm{L} \mathrm{T} 3$ and $1 \mathrm{mmol} / \mathrm{L}$ creatine were added to the maturation medium (MM-TC, Table 2).

\subsubsection{Cryopreservation of human PSC-derived skeletal myocytes}

Human PSC-derived skeletal myocytes were cryopreserved for long term storage. On day 22 of differentiation, cell culture was rinsed once with PBS (Thermo Fisher Scientific). TrypLE (Thermo Fisher Scientific) was added to the cells and incubated for 5 to 7 minutes at $37{ }^{\circ} \mathrm{C}$ and $5 \% \mathrm{CO}_{2}$. TrypLE digestion was stopped using expansion medium with $5 \mu \mathrm{mol} / \mathrm{L}$ Y27632 (Stemgent). Cell suspension was triturated very gently with a $10-\mathrm{ml}$ serological and centrifuged with $100 \mathrm{xg}$ at 20 $24^{\circ} \mathrm{C}$ for 10 minutes. Supernatant was removed and the pellet was resuspended very gently in freezing medium which contained cold expansion medium $\left(4^{\circ} \mathrm{C}\right)$ with $5 \mu \mathrm{M}$ Y27632 (Stemgent) 
and 10\% DMSO (Sigma-Aldrich). 10x10 $10^{6}$ human PSC-derived skeletal myocytes were frozen in $1.5 \mathrm{ml}$ freezing medium per cryovial in a MrFrosty ${ }^{\mathrm{TM}}$ freezing container (Nalgene) at $-80^{\circ} \mathrm{C}$ overnight and then stored at $-150^{\circ} \mathrm{C}$ (SANYO, Ewald Innovationstechnik GmbH).

\subsubsection{Thawing of human PSC-derived skeletal myocytes}

A frozen cryovial was taken from $-150^{\circ} \mathrm{C}$ freezer (SANYO, Ewald Innovationstechnik GmbH) and quickly thawed in water bath at $37^{\circ} \mathrm{C}$ for approximately 2 min until a small ball of ice was still visible in the thawing medium. The cryovial was sprayed with alcohol and taken under the laminar flow hood. Using a $2 \mathrm{ml}$ serological pipette, the contents of the cryovial were transferred to a preprepared $15 \mathrm{ml}$ tube containing $9 \mathrm{ml}$ of expansion medium with $5 \mu \mathrm{M}$ Y27632 (Stemgent). The cell suspension was centrifuged at $100 x g, 10$ minutes at $20-24^{\circ} \mathrm{C}$. Supernatant was removed and the pellet was resuspended very gently in expansion medium with $5 \mu \mathrm{M}$ Y27632 (Stemgent) for plating.

\subsection{Human engineered skeletal muscle}

\subsubsection{Preparation of casting molds and static stretchers}

For the generation of 3D muscle poly-dimethylsiloxane (PDMS; SYLGARD ${ }^{\mathrm{TM}} 184$ Silicone Elastomer Kit, Dow Corning) circular molds with inner/outer diameter 4/6 mm and $2.5 \mathrm{~mm}$ height were fabricated and allowed to cure overnight at $55^{\circ} \mathrm{C}$. Static stretch devices were made from a

Teflon ${ }^{\circledR}$ base and stainless steel holders. The detailed protocol for the preparation of the casting molds and static stretchers has been described previously (Soong, Tiburcy, and Zimmermann 2012; Tiburcy et al. 2014).

\subsubsection{Generation of human bioengineered skeletal muscle (BSM) organoids}

To make bioengineered skeletal muscle (BSM) organoids undifferentiated human iPSC were dissociated according to section 2.1.2 when the monolayer cell culture reached a confluency of 80$90 \%$. A final $250 \mu \mathrm{l} / \mathrm{BSM}$ hydrogel mixture of i) $0.23 \mathrm{mg}$ acid soluble collagen type 1 (Collagen 
Solutions), ii) concentrated 2x DMEM (Thermo Fisher Scientific) serum-free medium ( $0.27 \mathrm{~g}$ DMEM powder in $10 \mathrm{ml}$ ddH2O), iii) $\mathrm{NaOH} 0.1 \mathrm{~N}$ (Carl Roth), iv) 10\% v/v Matrigel ${ }^{\mathrm{TM}}$ (BD) and v) $0.8 \times 10^{6}$ iPSC resuspended in StemMACS iPS-Brew XF (Miltenyi Biotec) medium with $5 \mu \mathrm{M}$ Y27632 (Stemgent), $10 \mathrm{ng} / \mathrm{ml}$ FGF-2 (Peprotech) and 10\% knockout serum replacement (ThermoFisher Scientific) was cast into circular polydimethylsiloxane (PDMS) molds (Table 3). After $1 \mathrm{~h}$ of condensation at $37^{\circ} \mathrm{C}$, BSMs were cultured in StemMACS iPS-Brew XF (Miltenyi Biotec) medium with $5 \mu \mathrm{M}$ Y27632 (Stemgent), 10 ng/ml FGF-2 (Peprotech) and 10\% knockout serum replacement (ThermoFisher Scientific). Following tissue compaction (typically after 24 to $48 \mathrm{hrs}$ ) BSMs were induced to skeletal muscle differentiation following the exact protocol for 2D monolayer cells (section 2.1.4). On day 22 of differentiation, BSM was loaded on static stretchers at $120 \%$ of slack length and cultured in maturation medium (Table 2) with the addition of 1 $\mathrm{mmol} / \mathrm{L}$ creatine monohydrate (Sigma-Aldrich) for 4 weeks.

Table 3. Composition of hydrogel master mix for BSM

\begin{tabular}{|l|c|c|}
\hline BSM number & $1 \mathrm{x}$ & unit \\
\hline \hline Acid soluble collagen type 1 $(6.5 \mathrm{mg} / \mathrm{ml})$ & 144 & $\mu \mathrm{l}$ \\
\hline 2x DMEM serum-free medium & 36 & $\mu \mathrm{l}$ \\
\hline $\mathrm{NaOH} 0.1 \mathrm{~N}$ & 6.75 & $\mu \mathrm{l}$ \\
\hline Matrigel & 25 & $\mu \mathrm{l}$ \\
\hline Cell suspension (including $\left.0.8 \times 10^{6} \mathrm{iPSC}\right)$ & 157.5 & $\mu \mathrm{l}$ \\
\hline \hline Total volume & 250 & $\mu \mathrm{l}$ \\
\hline
\end{tabular}

\subsubsection{Generation of human engineered skeletal muscle (ESM)}

To generate human engineered skeletal muscle (ESM), either PSC-derived skeletal myocytes were dissociated, or frozen PSC-derived skeletal myocytes were thawed as described in section 2.1.8. A final $250 \mu \mathrm{l} / \mathrm{ESM}$ hydrogel mixture of i) $0.23 \mathrm{mg}$ acid soluble collagen type 1 (Collagen Solutions), ii) concentrated 2x DMEM (Thermo Fisher Scientific) serum-free medium ( $0.27 \mathrm{~g}$

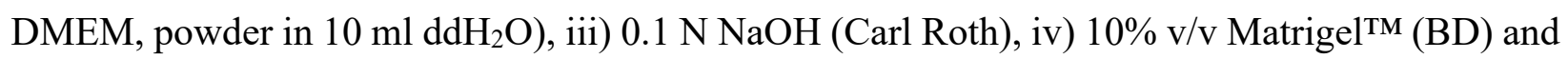
v) $1.25 \times 10^{6}$ of day $22 \mathrm{hPSC}$-derived skeletal myocytes resuspended in expansion medium with 5 $\mu \mathrm{M}$ Y27632 (Stemgent), was cast into circular PDMS molds (Table 4). After $1 \mathrm{~h}$ of condensation 
at $37^{\circ} \mathrm{C}$, ESMs were cultured in expansion medium with $5 \mu \mathrm{mol} / \mathrm{L}$ Y27632 (Stemgent) for $24 \mathrm{~h}$ and then expansion medium for another 6 days to consolidate into mechanically stable tissue. Medium was changed every second day and subsequently ESMs were transferred onto static stretchers to exert isometric load at $120 \%$ of the slack length. ESMs were cultured in maturation medium (Table 2) under mechanical load for up to 9 weeks. Maturation medium was changed every second day. Where indicated $1 \mathrm{mmol} / \mathrm{L}$ creatine monohydrate (Sigma-Aldrich) and 0.1 $\mu \mathrm{mol} / \mathrm{L}$ T3 were added to maturation medium from week 4 to 9 (MM-TC, Table 2).

Table 4. Composition of hydrogel master mix for ESM

\begin{tabular}{|l|c|c|}
\hline ESM number & $1 \mathrm{x}$ & unit \\
\hline \hline Acid soluble collagen type $1(6.5 \mathrm{mg} / \mathrm{ml})$ & 144 & $\mu 1$ \\
\hline $2 \mathrm{x}$ DMEM serum-free medium & 36 & $\mu 1$ \\
\hline $\mathrm{NaOH} 0.1 \mathrm{~N}$ & 6.75 & $\mu 1$ \\
\hline Matrigel & 25 & $\mu 1$ \\
\hline Cell suspension (including $1.25 \times 10^{6}$ skeletal myocytes) & 157.5 & $\mu 1$ \\
\hline \hline Total volume & 250 & $\mu 1$ \\
\hline
\end{tabular}

\subsubsection{Cardiotoxin injury model}

To induce muscle injury ESM were incubated in maturation medium with $25 \mu \mathrm{g} / \mathrm{ml}$ of Naja pallida cardiotoxin (CTX; Latoxan) for 24 h (Tiburcy et al. 2019). Subsequently the injured tissue was rinsed and cultured in expansion medium (Table 2) for 1 week and then switched to maturation medium with $1 \mathrm{mmol} / \mathrm{L}$ creatine. Medium was refreshed every second day.

To arrest cell cycle activity ESMs were irradiated with 30 Gy using an STS Biobeam 8000 (Germany) gamma irradiation $24 \mathrm{hrs}$ before CTX injury. 


\subsection{Immunostaining and confocal imaging}

$2 \mathrm{D}$ cell cultures were fixed in $4 \%$ formalin (Carl Roth) at $20-24^{\circ} \mathrm{C}$ for $15 \mathrm{~min}$. ESM/BSM were fixed in $4 \%$ formalin at $4^{\circ} \mathrm{C}$ overnight. For dystrophin staining, ESMs were fixed in ice-cold $100 \%$ acetone for $10 \mathrm{~min}$. After 2 washes with PBS, ESM/BSM were dehydrated in 70\% ethanol at 20$24^{\circ} \mathrm{C}$ for $1 \mathrm{~min}$ and then embedded in $2 \%$ agarose (peqGOLD) in $1 \mathrm{X}$ Tris Acetate-EDTA (TAE) buffer. Using a vibratome (Leica VT1000S), $400 \mu \mathrm{m}$ sections were cut and kept in $4^{\circ} \mathrm{C} 1 \mathrm{X}$ PBS. Prior to staining, 2D cell cultures as well as ESM sections were washed with 1X PBS followed by a blocking step to reduce unspecific binding using staining buffer (1X PBS with 5\% fetal bovine serum, $1 \%$ bovine serum albumin (BSA), and 0.5\% Triton-X). The following antibodies were applied for primary staining in staining buffer at $20-24^{\circ} \mathrm{C}$ for $4 \mathrm{hrs}$ (Appendix, Table 3): Oct4 (1:500, Abcam), Pax3-concentrate (1:100, DSHB), Pax7-concentrate (1:100, DSHB), MyoD (1:100, Dako) and Myogenin-concentrate (1:10, DSHB), Sarcomeric $\alpha$-actinin (1:500, SigmaAldrich), Laminin (1:50, Sigma-Aldrich), Dystrophin (1:500, Millipore), neurofilament H, SMI32 (1:20000, Biolegend), Beta-dystroglycan (1:50, LCL-b-DG, Leica Biosystem) and Ki67 (1:100, Abcam). After 3x PBS washes for 5 minutes, the appropriate Alexa Fluor-coupled secondary antibodies (1:1000, Thermo Fisher Scientific) were applied for $2 \mathrm{~h}$ at $20-24^{\circ} \mathrm{C}$. In parallel with secondary antibodies, Alexa Fluor 633-conjugated phalloidin (1:100, Thermo Fisher Scientific), Alexa Fluor 594-conjugated $\alpha$-Bungarotoxin and Hoechst 33342 (1:1000, Molecular Probes) were added to stain f-actin and nuclei, respectively. Following 3 washes with PBS, samples were mounted in Fluoromount-G (Southern Biotech). All images were acquired using a Zeiss LSM 710/NLO confocal microscope. To quantify the labeled cells, 3 random focal planes per sample from 3 different experiments were chosen for analysis with ImageJ cell counter tool.

\subsection{Flow cytometry}

After cell fixation in $4 \%$ formalin (Carl Roth) at $20-24^{\circ} \mathrm{C}$ for $15 \mathrm{~min}$ and $2 \mathrm{X}$ washes with PBS, samples were kept on ice for the staining process. To block unspecific binding, cells were incubated in staining buffer (1X PBS with 5\% fetal bovine serum, 1\% bovine serum albumin (BSA), and 0.5\% Triton-X) for 10 min. Cells were stained for Pax7 (1:500, DSHB), MyoD (1:500, Dako) and Myogenin (1:50, DSHB) and Sarcomeric $\alpha$-actinin (1:4000, Sigma-Aldrich), or 
appropriate isotype controls (R\&D systems) for $45 \mathrm{~min}$. Following two washes with PBS secondary antibodies (1:1000, Thermo Fisher Scientific) were applied for $30 \mathrm{~min}$. Samples were stained with Hoechst-3342 for nuclear DNA counting and exclusion of cell doublets. Cells were run on a LSRII SORP cytometer and at least 10,000 events per sample were analyzed using Diva software (BD Biosciences).

\subsection{RNA sequencing}

RNA was purified using Trizol (Thermo Fisher Scientific) according to the manufacturer's instructions. RNA quality was ensured with the Fragment Analyzer from Advanced Analytical by using the standard sensitivity RNA Analysis Kit (DNF-471). RNA-seq libraries were prepared using a modified strand-specific, massively parallel cDNA sequencing (RNA-Seq) protocol from Illumina, the TruSeqStranded Total RNA. Libraries were sequenced on a HiSeq4000 platform (Illumina) generating $50 \mathrm{bp}$ single end reads (30-40 Mio reads/sample). Sequence images were transformed with Illumina software BaseCallerto BCL files, which was demultiplexed to fastq files with bcl2fastq v2.17.1.14. The quality check was done using Fast QC (version 0.11.5, Babraham Bioinformatics). Sequence reads were aligned to the human genome reference assembly (UCSC version hg38) using Star. For each gene, the number of mapped reads was counted using Feature Counts. Raw counts were normalized and transformed to log2CPM values. Reads Per Kilobase per Million mapped reads (RPKM) were calculated based on Ensembl transcript length using biomaRT (v2.24). RNA sequencing was performed by the NGS Integrative Genomics (NIG) Core Unit.

\subsubsection{Bioinformatic analyses}

Weighted gene co-expression network analysis was performed by Dr. R. Islam, Fischer lab,

Department for Epigenetics and Systems Medicine in Neurodegenerative Diseases, using (WGCNA) package (version 1.61) in R. Briefly, normalized counts were transformed into log (base 2) counts and were used to calculate pairwise bi-weighted mid-correlations between genes. Next, based on approximate scale-free topology a soft threshold power of 14 was chosen and was 
used to calculate pair-wise topological overlap between genes to construct a signed gene coexpression network. Modules of co-expressed genes was later identified based on following criteria: minimum module size of 100 , method = "hybrid", deepSplit=0, pamRespectsDendro=T, pamStage $=\mathrm{T}$. Modules with correlation higher than 0.85 were merged together. Different modules were summarized as modular eigengenes (MEs), those were then used to compare expression of the given module across differentiation time points. The module specific genes were further filtered based on a module membership correlation coefficient cutoff of 0.60 . Gene ontology of the modules were analyzed using cluster Profiler (v3.0.4) and after multiple adjustments only statistically significant gene ontology terms (FDR $<0.05)$ were retrieved. For pathway analysis, Reactome (https://reactome.org/) database was used. To compare to human embryo data sets raw data from the study of (Xi et al. 2017) was retrieved from NCBI GEO (accession: GSE90876). Briefly, sequencing reads were mapped to human genome hg38 using STAR aligner (v2.5.2b). After mapping, raw count files were generated using featureCounts of subread package (v1.5.1). For differential expression analysis, all samples were processed together and genes with less than 5 reads in $50 \%$ of the samples were filtered out prior to the analysis. Differential expression analysis was performed using DESeq2 package (version 1.28.1) in R. Genes with FDR $<0.05$ were considered as differentially expressed. To test above chance overlap between previously identified module and differentially expressed genes, Fisher's exact test was performed.

\subsection{Single cell transcriptomics by single nuclei RNA sequencing}

Single nuclei were isolated from flash frozen cells. The cell pellet was homogenized using a plastic pestle in a $1.5 \mathrm{ml}$ Eppendorf tube containing $500 \mu \mathrm{l}$ EZ prep lysis buffer (Sigma, NUC101-1KT) with 30 strokes. The homogenate was transferred into $2 \mathrm{ml}$ microfuge tubes, lysis buffer was added up to $2 \mathrm{ml}$ and incubated on ice for 7 minutes. After centrifuging for 5 minutes at 500xg supernatant was removed and the nuclear pellet was resuspended into $2 \mathrm{ml}$ lysis buffer and incubated again on ice ( 7 minutes). After centrifuging for 5 minutes at $500 \mathrm{xg}$, the supernatant was removed and the nuclei pellet was resuspended into $500 \mu 1$ nuclei storage buffer (NSB: 1x PBS; Invitrogen, 0.5\% RNase free BSA;Serva, 1:200 RNaseIN plus inhibitor; Promega, 1x EDTA-free protease inhibitor; Roche) and filtered through $40 \mu \mathrm{m}$ filter (BD falcon) with additional $100 \mu \mathrm{L}$ NSB to collect residual nuclei from the filter. Isolated nuclei were stained with a nuclear stain (7AAD) and FACS 
sorted (BD FACSaria III) to ensure a homogenous and viable nucleus preparation. Sorted nuclei were counted in a Countess FL II automated cell counter (ThermoFischer AMQAF1000, DAPI light cube: ThermoFischer: AMEP4650) with DAPI staining and nuclei concentration was adjusted to 1000 nuclei/ $\mu \mathrm{L}$. The nuclei were further diluted to capture and barcode 4000 nuclei according to Chromium single cell 3' reagent kit v3.1 (10X Genomics). Single nuclei barcoding, GEM formation, reverse transcription, cDNA synthesis and library preparation were performed according to $10 \mathrm{X}$ Genomics guidelines. cDNA libraries were pooled and sequenced 8 times in Illumina NextSeq 550 in order to achieve the target reads / nuclei. Each sequencing run was acquiring 150bp paired-end reads (Illumina NextSeq 550 High Output Kit v2.5). Demultiplexing, read mapping (to pre-mRNA reference genome) and gene counts per nuclei were computed with cellranger (v4.0) software. The nuclei barcoding and sequencing pipeline typically allows to obtain 50.000-100.000 reads/nucleus resulting in detection of 200-10.000 genes/nucleus (median: 2000 genes/nucleus) for further downstream analysis These analyses were done in collaboration with S. Sadman, Fischer lab, Department for Epigenetics and Systems Medicine in Neurodegenerative Diseases.

\subsubsection{Bioinformatic analysis of single-nucleus RNA-sequencing}

Gene counts were obtained by aligning reads to the hg38 genome (NCBI:GCA 000001405.22) (GRCh38.p7) using CellRanger software (v.3.0.2) (10XGenomics). The CellRanger count pipeline was used to generate a gene-count matrix by mapping reads to the pre-mRNA as reference to account for unspliced nuclear transcripts. The SCANPY package was used for pre-filtering, normalization and clustering (Wolf, Angerer, and Theis 2018). Initially, cells that reflected lowquality cells (based on read number and expression of house-keeping genes (Eisenberg and Levanon 2013)) were excluded. Next, counts were scaled by the total library size multiplied by 10.000, and transformed to log space. Highly variable genes were identified based on dispersion and mean, the technical influence of the total number of counts was regressed out, and the values were rescaled. Principal component analysis (PCA) was performed on the variable genes, and UMAP was run on the top 50 principal components (PCs) (Becht et al. 2018). The top 50 PCs were used to build a k-nearest-neighbours cell-cell graph with k=100 neighbors. Subsequently, spectral decomposition over the graph was performed with 50 components, and the Leiden graph- 
clustering algorithm was applied to identify cell clusters. We confirmed that the number of PCs captures almost all the variance of the data. For each cluster, we assigned a cell-type label using manual evaluation of gene expression for sets of known marker genes. A muscle gene panel (Appendix) was identified by calculating the differentially expressed genes between myogenic and non-muscle cluster with a low frequency cutoff of 1 and an adjusted $p$ value of $<0.05$. These analyses were done by D. Krüger, Fischer lab, Department for Epigenetics and Systems Medicine in Neurodegenerative Diseases.

\subsection{Isometric force measurements}

Contractile function of engineered muscle was measured under isometric conditions in a thermostatted organ baths (Föhr Medical Instruments) filled with gassed $\left(5 \% \mathrm{CO}_{2} / 95 \% \mathrm{O}_{2}\right)$ Tyrode solution (containing: $120 \mathrm{NaCl}, 1 \mathrm{MgCl} 2,0.2 \mathrm{CaCl} 2,5.4 \mathrm{KCl}, 22.6 \mathrm{NaHCO}$, 4.2 NaH2PO4, 5.6 glucose, and 0.56 ascorbate; all in $\mathrm{mM}$ ) at $37^{\circ} \mathrm{C}$. The calcium concentration was set to $1.8 \mathrm{mM}$. To adjust the muscle length to maximal force production ESMs were electrically stimulated at 1 $\mathrm{Hz}$ with $4 \mathrm{~ms}$ square pulses of $200 \mathrm{~mA}$ and the muscle length was step-wise increased by intervals of $125 \mu \mathrm{m}$ until the maximum twitch force was observed. At the length of maximal force generation, tetanic twitch tension was assessed at higher frequency stimulations (stimulation at 5 , $10,20,40,60,80$ and $100 \mathrm{~Hz}$ for 4 seconds). Activation of nicotinic acetylcholine receptors was investigated by adding the unspecific cholinergic receptor agonist carbachol $(1 \mu \mathrm{M})$. Contraction data was recorded with BMON software and analyzed using AMON software (Ingenieurbüro Jäckel).

\subsection{Western blot analysis}

For protein isolation ESMs were placed in Eppendorf tubes and snap frozen in liquid nitrogen. 150 $\mu 1$ of ice-cold protein lysate buffer (2.38 g HEPES, $10.20 \mathrm{~g} \mathrm{NaCl}, 100 \mathrm{ml}$ Glycerol, $102 \mathrm{mg} \mathrm{MgCl}$, $93 \mathrm{mg}$ EDTA, $19 \mathrm{mg}$ EGTA, $5 \mathrm{ml} \mathrm{NP}-40$ in a total volume of $500 \mathrm{ml} \mathrm{ddH2O}$ ) containing PhosSTOP ${ }^{\circledR}$ phosphatase inhibitor and cOmplete ${ }^{\circledR}$ protease inhibitor mix (Roche) was added to the ESM. A $7 \mathrm{~mm}$ stainless steel bead (Qiagen) was placed in the Eppendorf tube and the sample 
was homogenized using TissueLyser (Qiagen) for 30 seconds at $30 \mathrm{~Hz}$ at $4^{\circ} \mathrm{C}$ and subsequently incubated on ice for 2 hours and then centrifuged for $30 \mathrm{~min}$ at $12000 \mathrm{rpm}$ at $4^{\circ} \mathrm{C}$. The supernatant was collected as protein sample and protein concentration was measured using the Bradford protein assay. $30 \mu \mathrm{g}$ of protein sample was loaded on a 4 to $15 \%$ gradient sodium dodecyl sulfate (SDS)-polyacrylamid gel (Bio-Rad), run at $100 \mathrm{~V}$ for approximately 2.5 hours followed by protein transfer to a polyvinylidene fluoride (PVDF) membrane at $30 \mathrm{~V}$ in an ice-filled box staying in cold room overnight. To visualize the total protein, the PVDF membrane was stained with Ponceau Red. Primary antibody $\left(4 \mathrm{~h}\right.$ at $\left.20-24^{\circ} \mathrm{C}\right)$ and secondary antibody $\left(1 \mathrm{~h}\right.$ at $\left.20-24^{\circ} \mathrm{C}\right)$ staining was performed in a staining buffer containing 5\% milk in $1 \mathrm{x}$ Tris-buffered saline (TBS) and $0.1 \%$ Tween 20. The following primary antibodies were applied: dystrophin monoclonal antibody (1:500, MANDYS8, Sigma-Aldrich), embryonic myosin heavy chain 3 (1:500, F1.652, DSHB), slow type myosin heavy chain 7 (1:500, A4.951, DSHB) and fast type myosin heavy chain 2 (1:100, A4.74, DSHB). Protein loading was controlled by Vinculin (VCL) antibody (1:5000, V3131, Sigma-Aldrich). The membrane was washed for 5 min with 1x Tris-buffered saline (TBS) and $0.1 \%$ Tween 20 . Horseradish peroxidase conjugated goat anti-mouse IgG antibody (1:10000, P0260, Dako) was used for the secondary staining. After washing the membrane for 5 min with 1x Tris-buffered saline (TBS) and $0.1 \%$ Tween 20 , the blot was developed using femtoLUCENT ${ }^{\mathrm{TM}}$ PLUS-HRP Chemiluminescent reagents (Gbiosciences) and the protein bands were imaged using the BIO-RAD ChemDoc ${ }^{\mathrm{TM}} \mathrm{MP}$ system. Signal quantification was performed using ImageJ.

\subsection{Transmission Electron Microscopy}

Ultrastructural analysis was performed in collaboration with Dr. A. Unger, Institute of Physiology II, on ESM samples fixed in $4 \%$ formalin (Carl Roth), 15\% saturated picric acid in $0.1 \mathrm{M} \mathrm{PBS}$, $\mathrm{pH} 7.4$, at $4{ }^{\circ} \mathrm{C}$ overnight. ESMs were rinsed twice in $\mathrm{PBS}$ and treated with $0.5 \% \mathrm{OsO}_{4}$ for $45 \mathrm{~min}$ following several washing steps in $100 \mathrm{mM}$ phosphate buffer. Samples were counterstained with uranyl acetate, dehydrated via ethanol series, and embedded in DurcupanACM epoxy resin (Sigma-Aldrich). Ultrathin sections were prepared from resin blocks using a Leica UltracutS ultramicrotome (Mannheim, Germany) and adsorbed to glow-discharged formvar-carbon-coated copper single-slot grids. Electron micrographs were recorded using a Zeiss LEO 910 electron 
microscope; images were taken with a TRS SharpeyeCCD camera (Troendle, Moorenweis, Germany).

\subsection{Statistical analysis}

All data were analyzed using GraphPad Prism 7 software (GraphPad Software Inc., San Diego) and presented as mean \pm standard error of the mean (SEM). Statistical analyses were done using unpaired, two-tailed, Student's t-test, one-way or two-way ANOVA where appropriate assuming equal variances. Significantly different variances were corrected for. Results showing $p<0.05$ were considered significant and $n$ indicates the number of samples. 


\section{Results}

\subsection{Directed differentiation of hPSCs into skeletal myocytes}

\subsubsection{Sequential recapitulation of key stages of skeletal muscle development}

To generate hPSC-derived skeletal myocytes specific signaling pathways were emulated to recapitulate the key stages of embryonic muscle development (Figure 8A). The first stage is the induction of paraxial mesoderm. In vitro, activation of Wnt and FGF signaling will push the PSC to the mesodermal fate and prevent formation of ectopic neural tissue (Boulet and Capecchi 2012; Chal et al. 2015). Parallel inhibition of BMP signaling will promote paraxial but not lateral plate mesoderm differentiation (Chal et al. 2018; Miura et al. 2006). Therefore, to robustly generate paraxial mesoderm within the first 4 days of differentiation hPSCs were treated with $10 \mu \mathrm{M}$ of glycogen synthase kinase 3 (GSK3) inhibitor CHIR99021 (canonical Wnt signaling activator (Naujok et al. 2014)), $10 \mathrm{ng} / \mathrm{ml}$ of FGF2 (FGF receptor agonist) and $0.5 \mu \mathrm{M}$ of BMP receptor inhibitor LDN-193189 (Boergermann et al. 2010). It was confirmed that this treatment induces expression of paraxial (TBX6) but not lateral plate mesoderm (MESP1) markers (Figure 8B). In the following developmental step, Notch signaling inhibition promotes the formation of dermomyotome from FOXC2-positive progenitors of the anterior region of presomitic mesoderm (aPSM). Parallel inhibition of BMP signaling in vivo will specifically form the epaxial dermomyotome expressing MYF5 which further develop into trunk muscle (Marcelle et al. 1997; Tajbakhsh and Spörle 1998). Since the aim was to direct the cultures towards hypaxial dermomyotomal progenitors expressing $S I X 1$, which will later give rise to the limb myogenic progenitors and not trunk, inhibition of BMP signaling was stopped while inhibition of Notch signaling was started with a gamma secretase inhibitor (DAPT, $10 \mu \mathrm{M}$ ) to specifically drive the aPSM cells towards dermomyotomal progenitors of the limb (F. Bladt et al. 1995; Buckingham and Relaix 2007; Mayeuf-Louchart et al. 2014). Since Notch activation increases the expression level of FOXC2 in aPSM progenitors which will pave the way for endothelial differentiation, inhibition of Notch signaling was also crucial to downregulate $F O X C 2$ and elevate the ratio of $P A X 3 / F O X C 2$ with a subsequent increase in the number of PAX3+ dermomyotomal progenitors (Delfini et al. 2000; Mayeuf-Louchart et al. 2014). Differentiation of the aPSM progenitors to dermomyotomal progenitors of limb was evident by downregulation of $F O X C 2$ and upregulation 
of $P A X 3$ from day 4 to day 13. (Figure 8C). In addition, the upregulation of $S I X 1$ in the absence of MYF5 clearly indicates formation of limb muscle (Figure 8C).

A

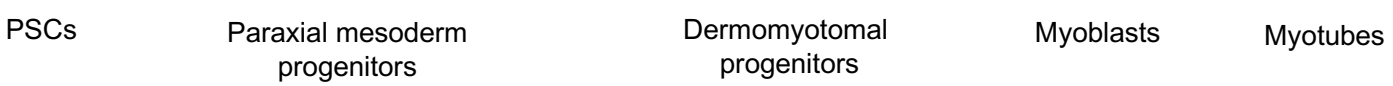

\section{POU5F1}

\section{TBX6}

FOXC2

PAX3/SIX1

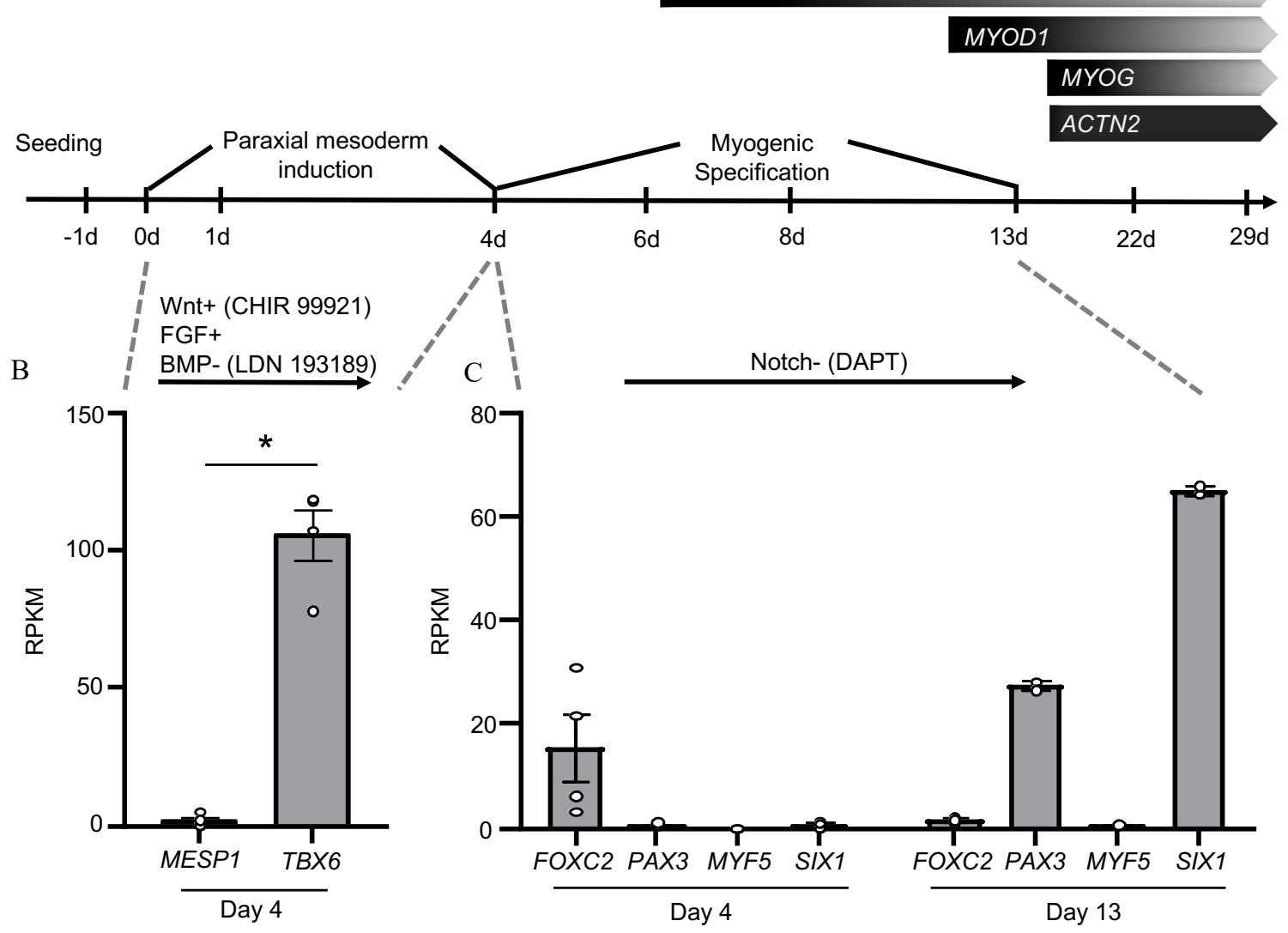

$\mathrm{D}$
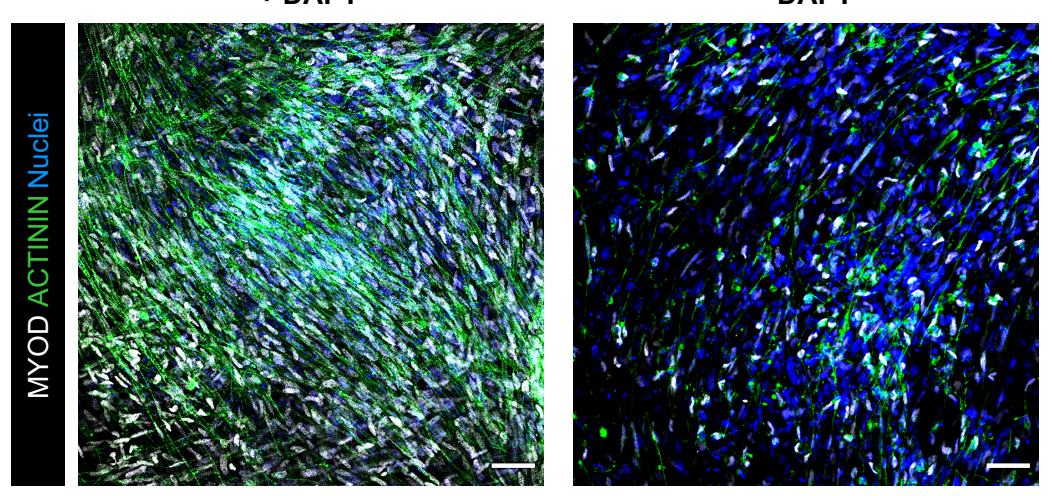


\section{Figure 8. Directed limb skeletal myogenesis in vitro.}

A, Diagram of key myogenic markers and a timeline of their approximate expression during directed differentiation of hPSCs into limb skeletal muscle cells, B, Specific paraxial but not lateral mesoderm induction in human PSCs. PSC cultures were treated with Wnt activator (10 $\mu \mathrm{M}$ CHIR99021), FGF receptor agonist (10 ng/ml FGF2) and BMP inhibitor $(0.5 \mu \mathrm{M}$ LDN-193189) from day 0 to day 4. Comparison of reads per kilobase million (RPKM) of signature genes of lateral mesoderm (MESP1) vs. paraxial mesoderm (TBXO) in PSC-derived presomitic progenitors (PSM) was performed on day 4 of differentiation; $\mathrm{n}=4$ /group; * $\mathrm{p}<0.05$ by Student's t-test. C, Expression (RPKM) of presomitic $(F O X C 2)$ and dermatomyotomal $(P A X 3, M Y F 5, S I X 1)$ progenitor genes in day $4(\mathrm{n}=4)$ and day $13(\mathrm{n}=2)$ cultures in the presence of Notch inhibitor DAPT. D, Impact of Notch inhibition on skeletal myocyte differentiation. Differentiating PSC cultures (HES2) were treated with Notch inhibitor (10 $\mu$ M DAPT) or without from day 4-day 13. Immunostaining of resulting skeletal myocytes was performed at day 22 for myogenic regulatory factor MYOD, sarcomeric $\alpha$-Actinin, and Nuclei (blue). Scale bars: $50 \mu \mathrm{m}$. ACTN2: Actinin alpha 2; FOXC2: Forkhead-related transcription factor 2; MYOD1: Myogenic differentiation 1; MYOG: Myogenin; PAX3: Paired box gene 3; POU5F1: POU Class 5 homeobox 1 (OCT4); SIX1: Sine oculis-related homeobox homolog 1; TBX6: T-box transcription factor 6.

The yield of skeletal myocytes at day 22 of differentiation in cultures treated with DAPT was clearly enhanced compared to cultures without Notch inhibition (Figure 8D). Considering that Notch inhibition prevents differentiation of PAX3+ cells to early myoblasts DAPT was stopped on day 12 of differentiation to stimulate the expression of myogenic regulatory factors (MRFs) such as PAX7, MYOD and Myogenin (Choi et al. 2016; Hirsinger et al. 2001).

During primary skeletal myogenesis of the limb, PAX3 increases the transcription of c-MET, a receptor tyrosine kinase that after binding of its ligand, hepatocyte growth factor (HGF), regulates growth and migration of myogenic precursor cells derived from dermomyotome. Furthermore, HGF signaling supports activation of MYOD expression and migration of myoblasts (Friedhelm Bladt et al. 1995; Buckingham and Relaix 2007; Mayeuf-Louchart et al. 2014). Consequently, 10 $\mathrm{ng} / \mathrm{ml}$ of HGF to stimulate c-MET signaling was applied from day 6 to 22 of differentiation. Fusion of MYOD expressing myoblasts into Myogenin+/Actinin+ myotubes was stimulated by using maturation medium from day 22 to 29 of differentiation.

The resulting final protocol is displayed in Figure 9A. To further characterize the specific stages of muscle development the expression patterns of key signature genes were analyzed. This confirmed the recapitulation of sequential distinct phases of myogenesis with loss of pluripotency (POU5F1), induction of paraxial mesoderm (MSGN1), generation of presomitic progenitors 
(MESP2), generation of dermomyotomal progenitors (PAX3, PAX7), and developing myoblasts (MYOD1, MYOG, ACTN2, DMD) (Figure 9B).

A
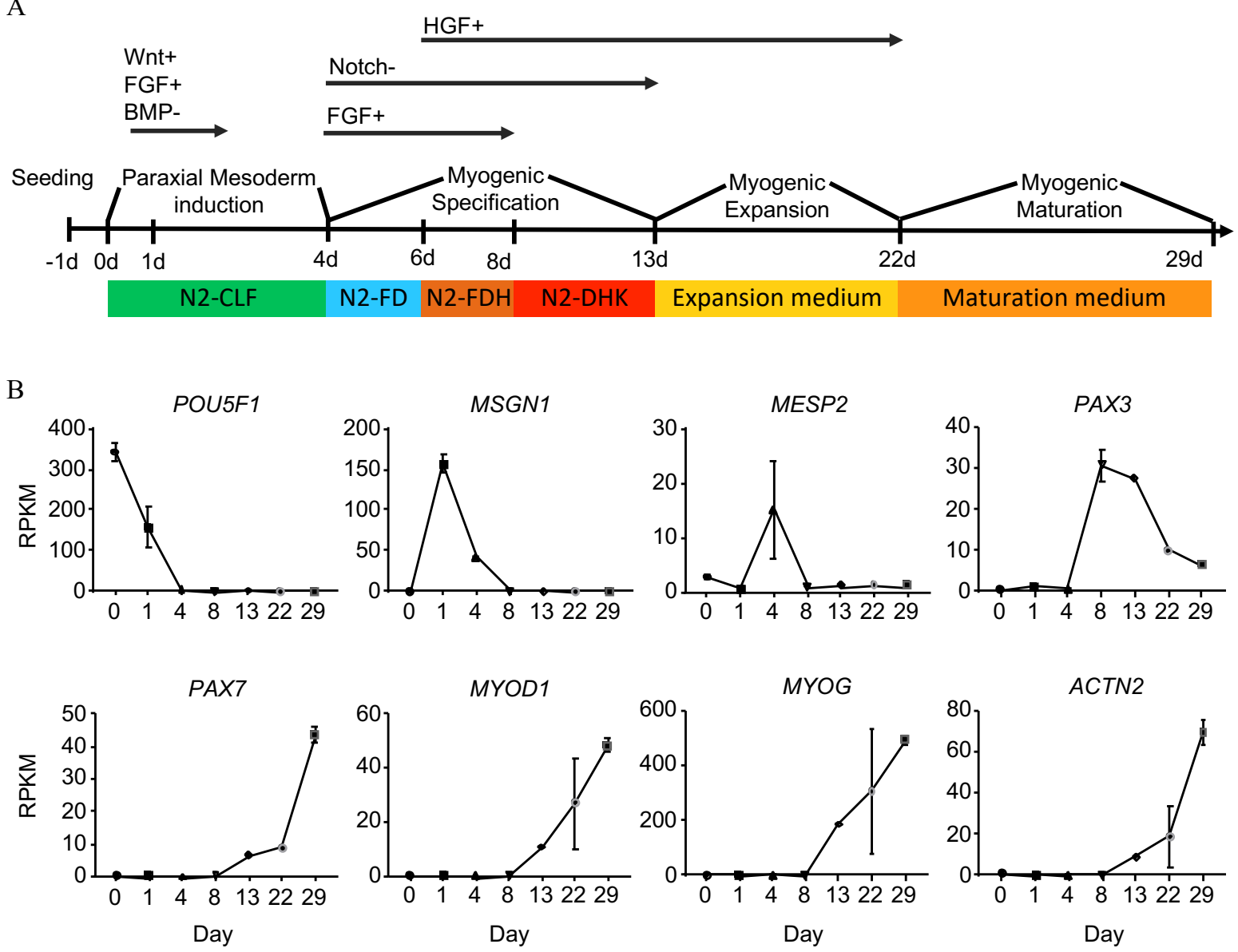

Figure 9. Recapitulation of sequential distinct phases of myogenesis during directed skeletal muscle differentiation from human PSCs.

A, Summary of the protocol for directed skeletal muscle differentiation from PSC indicating the sequence and the timing of factor addition to modulate specific signaling pathways involved in skeletal myogenesis. $+/-$ indicates pathway activation/inhibition, respectively. B, Reads Per Kilobase Million (RPKM) of signature genes for pluripotency ( $P O U 5 F 1)$, paraxial mesoderm (MSGN1), somitogenesis (MESP2), dermomyotome formation (PAX3), myogenic regulatory factors $(P A X 7, M Y O D$ and $M Y O G)$ and structural assembly (ACTN2), during skeletal muscle differentiation from human PSCs; $\mathrm{n}=2-4 /$ time point. ACTN2: Actinin alpha 2; MESP2: Mesoderm posterior BHLH transcription factor 2; MSGN1: Mesogenin 1; MYOD1: Myogenic differentiation 1; MYOG: Myogenin; PAX3: Paired box gene 3; PAX7: Paired box gene 7; POU5F1: POU class 5 homeobox 1 (OCT4).

This sequence of muscle developmental steps was also confirmed on protein level. In accordance with the transcriptome data, immunostaining during 29 days of skeletal muscle differentiation demonstrated OCT4+ pluripotent stem cells on day 1, expansion of PAX3+ early somite cells on 
day 8 and expression of PAX7+ myogenic progenitors in parallel with alignment of fusioncompetent myoblasts expressing sarcomeric $\alpha-$ ACTININ, MYOD1 and MYOGENIN initiated on day 13 and enhanced by day 22 and day 29 (Figure 10).

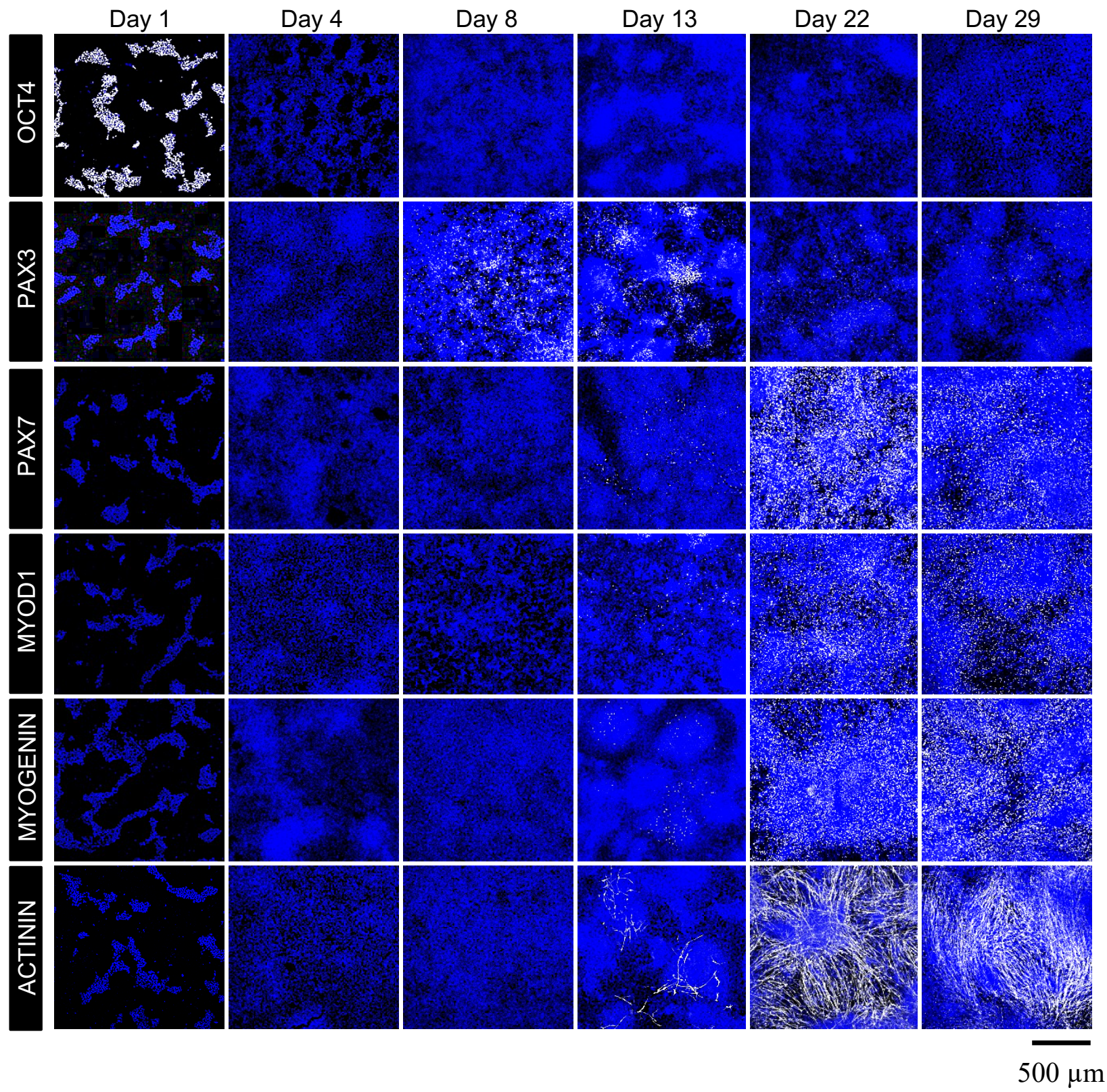

Figure 10. Development of key marker proteins during directed skeletal muscle differentiation of hPSCs. Immunostaining of OCT4, PAX3, PAX7, MYOD1, MYOGENIN, sarcomeric $\boldsymbol{\alpha}$-ACTININ (in gray), and Nuclei (blue) at indicated time points of skeletal muscle differentiation from TC1133 line (iPSC 1). Scale bar: $500 \mu \mathrm{m}$. 


\subsubsection{Evaluation of skeletal muscle differentiation efficiency}

To characterize the differentiated cultures the expression of bona-fide skeletal myogenic markers was quantified by immunostaining (Figure 11A). Differentiated cultures at day 22 contained a myogenic cell population consisting of $43 \pm 4 \%$ PAX7+, $52 \pm 2 \%$ MYOD $1+$, and $49 \pm 4 \%$ MYOGENIN+ cells (Figure 11B).

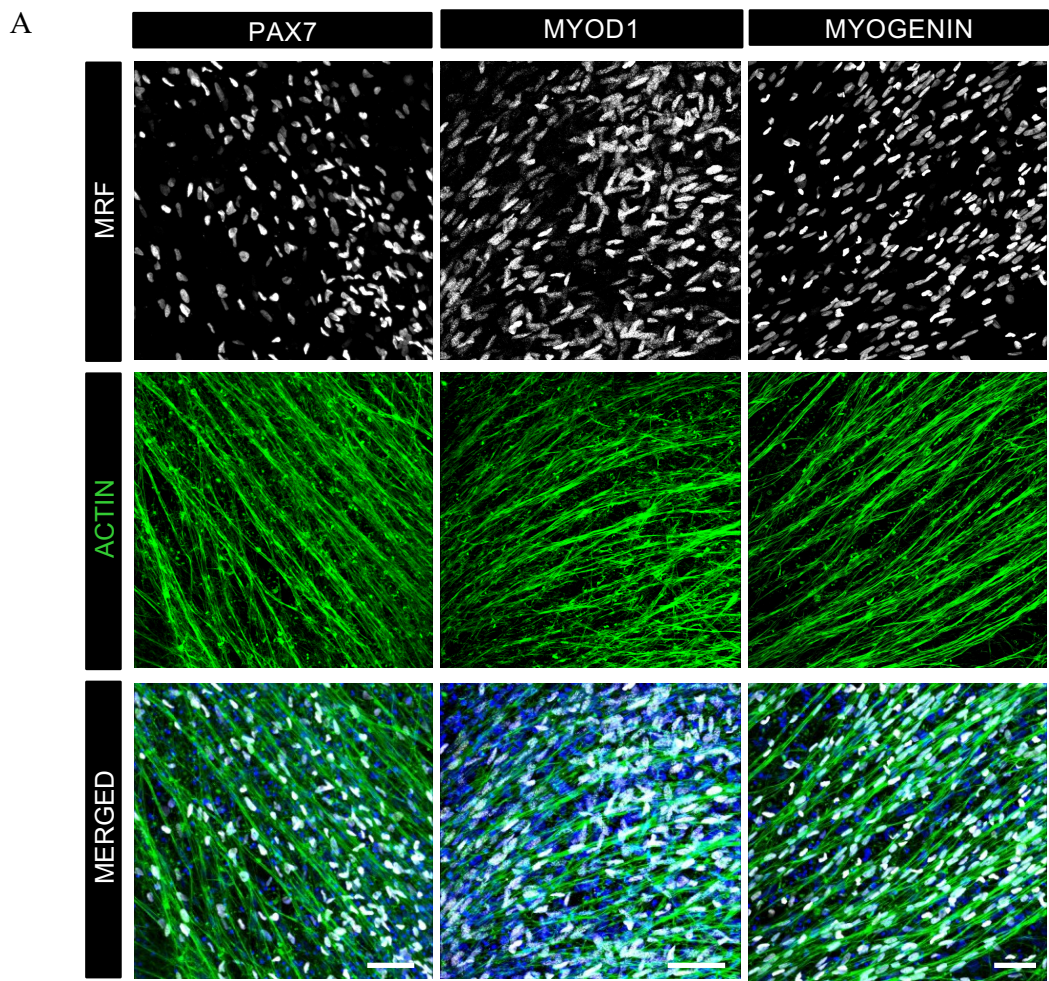

B

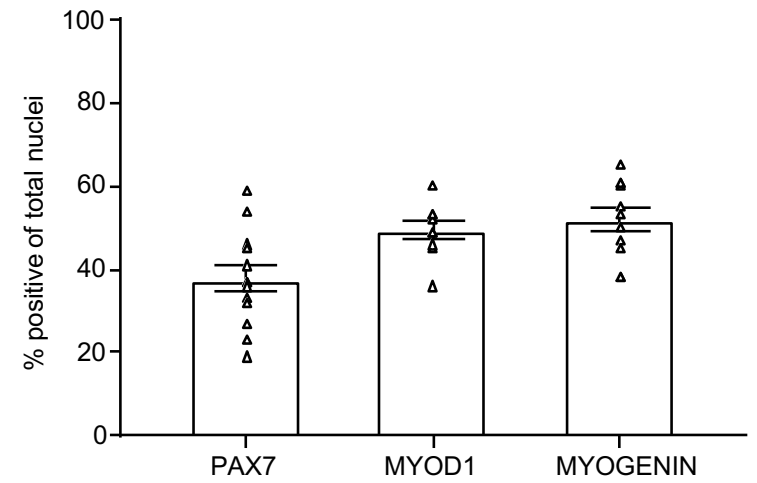

Figure 11. Efficient differentiation of hPSCs into skeletal muscle cell populations.

A, Immunostaining for myogenic regulatory factors: PAX7 (left, gray), MYOD1 (middle, gray), MYOGENIN (right, gray), f-ACTIN (green) and Nuclei (blue) in 22 days old skeletal muscle culture from TC1133 (iPSC 1) line; Scale bars: $50 \mu \mathrm{m}$. B, Quantification of nuclei positive for PAX7, MYOD1 and MYOGENIN in 22 day old myogenic cultures from HES2 and from TC1133 (iPSC 1) lines; $\mathrm{n}=9-13$. 
These data were confirmed by flow cytometry for nuclear myogenic regulatory factors on day 22 differentiated cultures. In line with the immunostaining results of adherent cultures the results were comparable with 45.4\% PAX7, 51.0\% MYOD1, 55.5\% MYOGENIN positive cells in the TC1133 (iPSC 1) line. Importantly, flow cytometry also confirmed a similar efficiency between multiple iPSC and ESC lines employed supporting the robustness and reproducibility of the protocol (Figure 12).

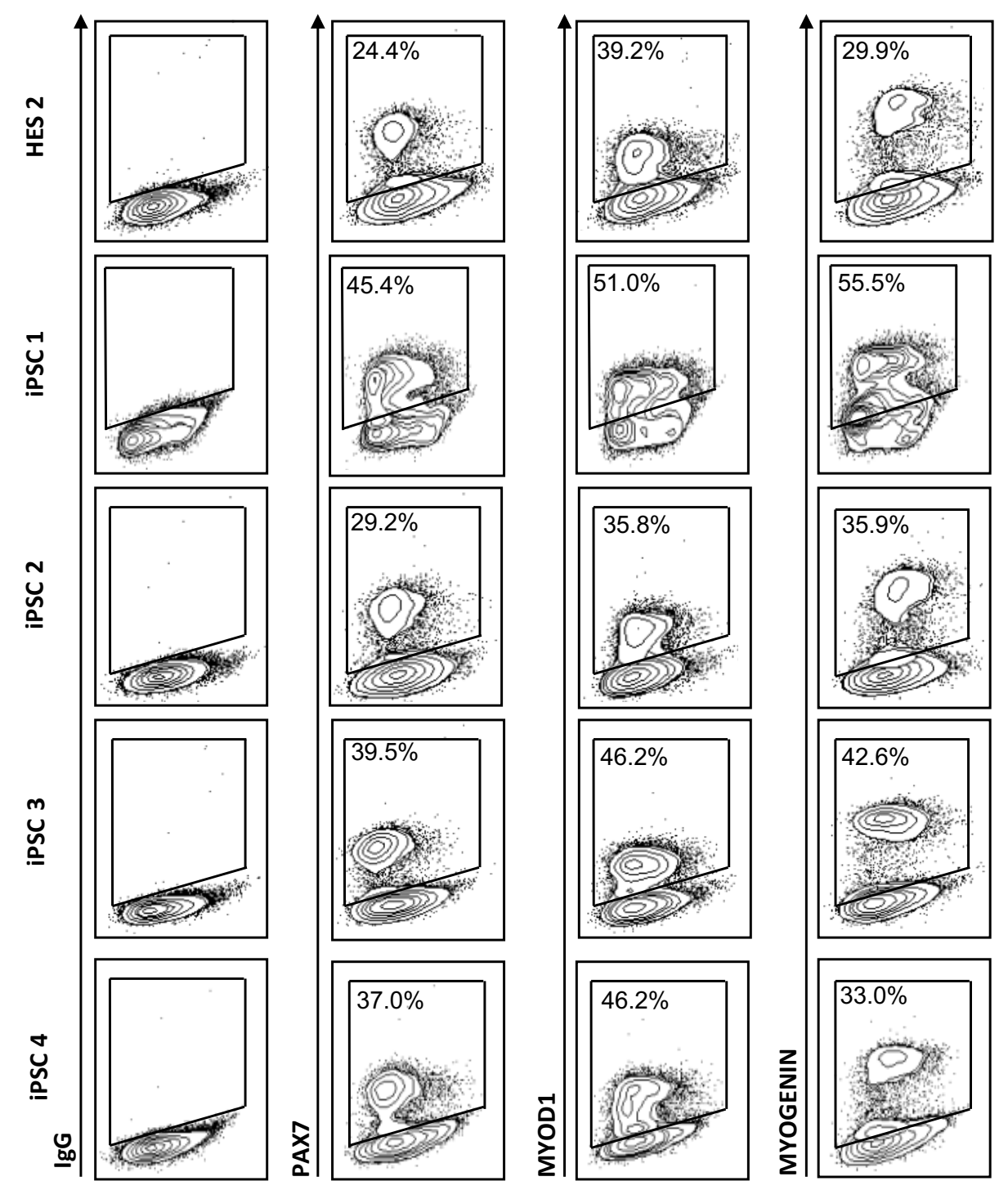

Figure 12. Robust skeletal muscle differentiation from multiple hPSCs lines.

Flow cytometry analysis of myogenic regulatory factors: PAX7, MYOD1 and MYOGENIN on 22 days old skeletal muscle differentiations from 5 human PSC lines. The percentage of cells positive for the respective marker is indicated. 


\subsubsection{Transcriptome profiling of skeletal muscle differentiation from hPSCs}

To further dissect the dynamic transcriptional landscape during skeletal myogenesis from hPSCs in vitro an RNA sequencing experiment was performed. RNA samples were collected on decisive time points of differentiation including day 0, 1, 4, 8, 13, 22, 29, and 60 (Figure 13A). Unbiased clustering of genes identified temporally distinct developmental gene expression patterns (Figure 13B). Interestingly, by clustering the genes according to their changes in time (weighted coexpression analysis) 22 gene clusters were identified of which some showed remarkable similarity to the biological processes of skeletal muscle differentiation in vivo (Figure 13C). 
A

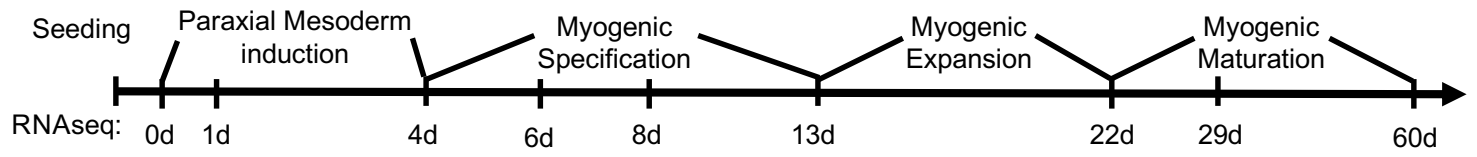

B

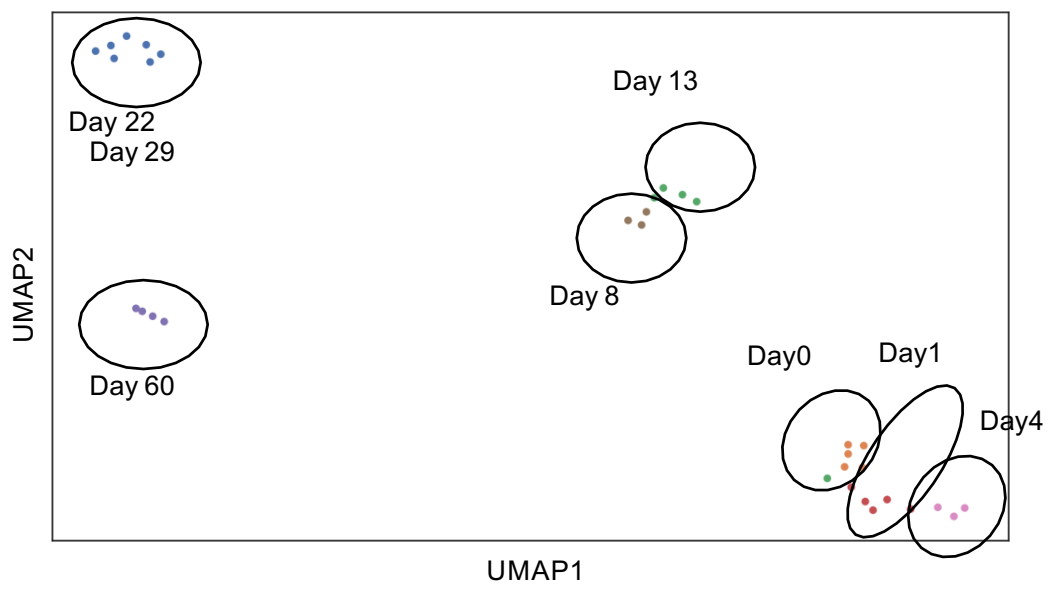

$\mathrm{C}$

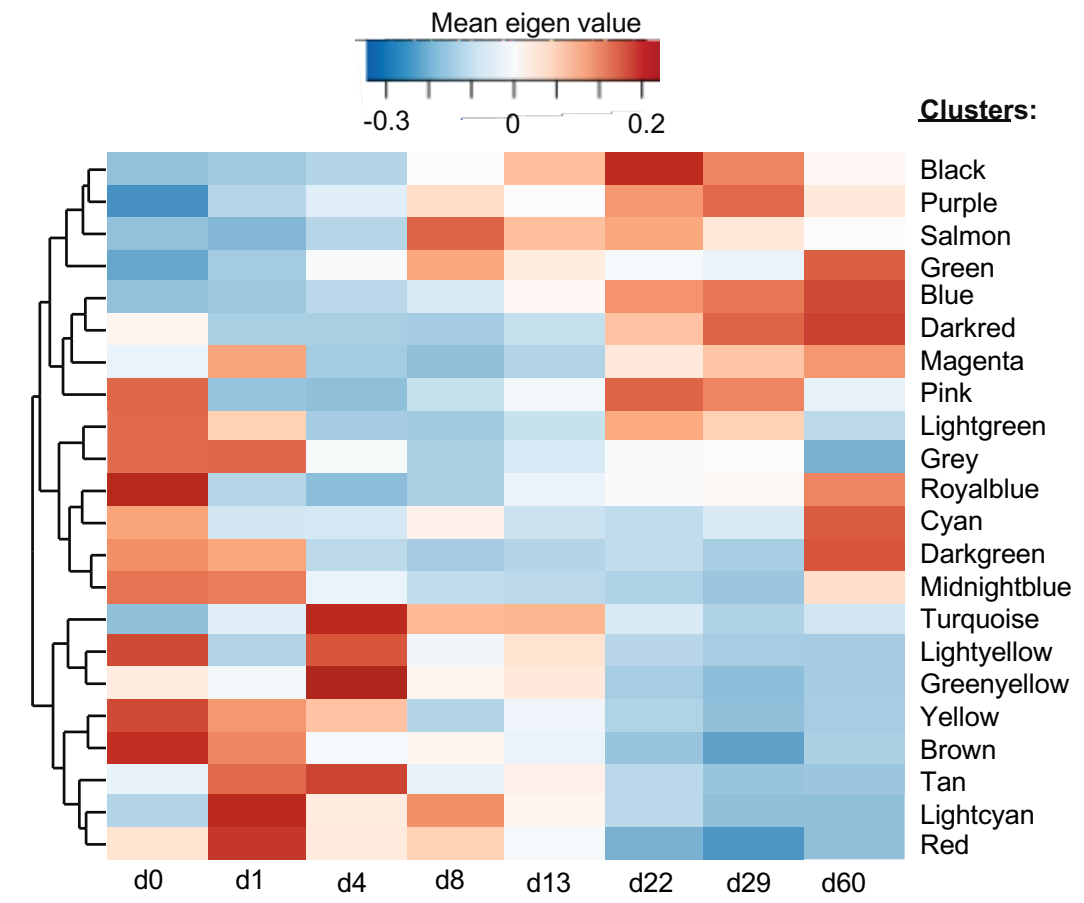

Figure 13. Unbiased transcriptome profiling identifies temporally distinct clusters of skeletal muscle development.

A, Scheme of skeletal muscle differentiation from hPSCs with sampling time points for RNA sequencing. B, Unsupervised clustering of the samples from different time points. C, Weighted coexpression analysis identified 22 cluster of genes with similar expression dynamics (coexpression clusters), heatmap of mean eigen values. Clusters are generically labeled by colors. Data obtained in collaboration with Dr. R. Islam, Fischer lab, Department for Epigenetics and Systems Medicine in Neurodegenerative Diseases. 
The unsupervised coexpression analysis confirmed the induction of paraxial mesoderm with a simultaneous decrease of pluripotency markers (POU5F1; cluster yellow) and a transient but robust increase in posterior (TBX6; cluster brown) and anterior (FOXC2; cluster turquoise) paraxial mesoderm markers. Interestingly, also segmentation genes like HES7 (cluster red) were activated in in vitro myogenesis (Figure 14).

A

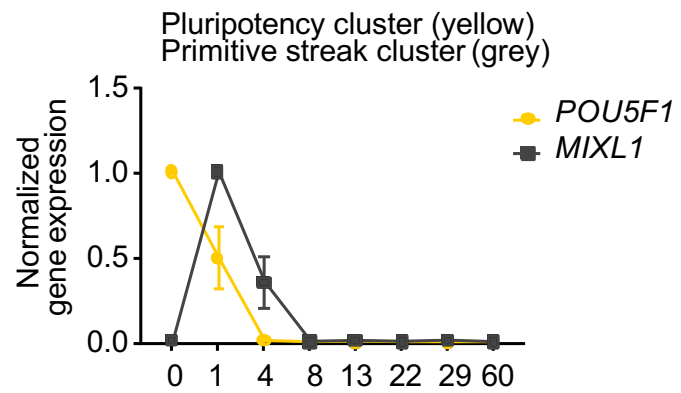

$\mathrm{C}$

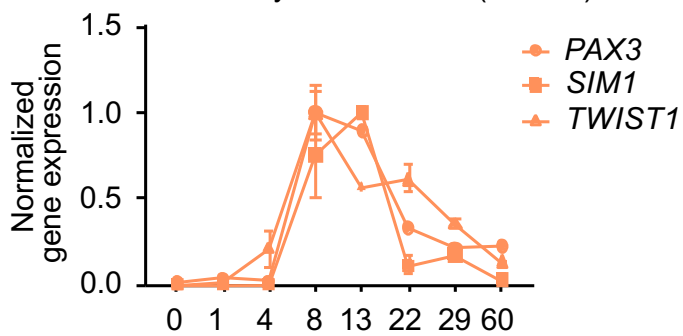

$\mathrm{E}$

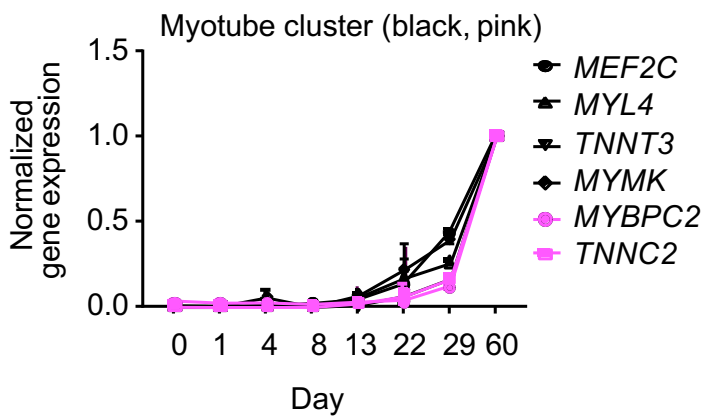

B Presomitic progenitor cluster

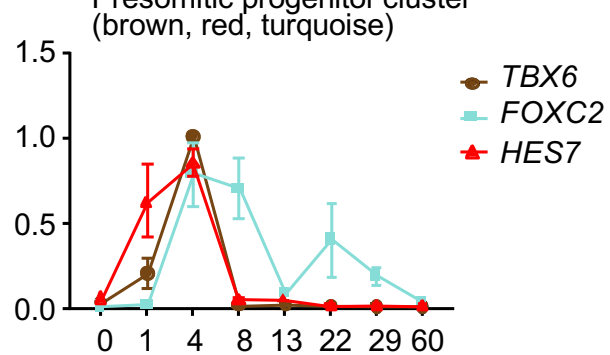

D Myoblast cluster (darkred)

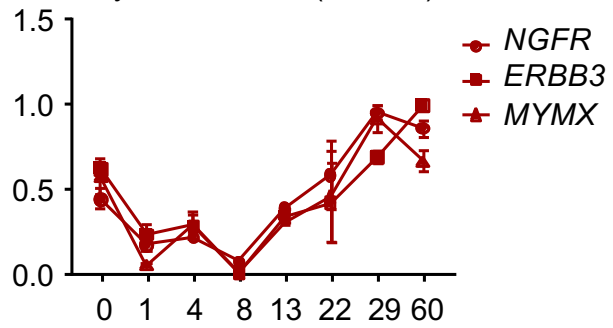

F Myotube maturation cluster (blue)

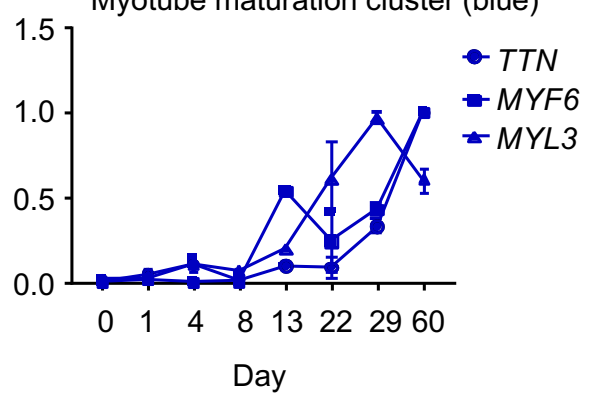

Figure 14. Signature gene expression in temporal coexpression clusters.

Normalized expression levels (RPKM) of indicated signature genes in identified coexpression clusters, $\mathrm{n}=2-4 /$ time point. ERBB3: Erb-B2 receptor tyrosine kinase 3; FOXC2: Forkhead-related transcription factor 2; HES7: Hes family BHLH franscription factor 7; PAX3: Paired box gene 3; POU5F1: POU Class 5 homeobox 1 (OCT4); MEF2C: Myocyte enhancer factor 2C; MIXL1: Mix paired-like homeobox; MYBPC2: myosin binding protein C2; MYF6: Myogenic factor 6; MYL3: Myosin light chain 3; MYL4: Myosin light chain 4; MYMK: Myomaker, myoblast fusion factor; MYMX: Myomixer, myoblast fusion factor; NGFR: Nerve growth factor receptor; SIM1: Sim BHLH transcription factor 1; TBX6: T-box transcription factor 6; TNNC2: Troponin C2, fast skeletal type; TNNT3: Troponin T3, fast skeletal type; TTN: Titin; TWIST1: Twist family BHLH transcription factor 1. Data obtained in collaboration with Dr. R. Islam, Fischer lab, Department for Epigenetics and Systems Medicine in Neurodegenerative Diseases. 
Dermomyotome specification was characterized by co-occurring expression of PAX3, SIMI and TWIST1 (cluster salmon) on day 13, followed by an increase in genes indicative of myogenesis (ERBB3 and NGFR) and fusion of myoblasts (MYMX; cluster darkred) to form myotubes (Figure 15). Further differentiation of myotubes was identified by expression of MEF2C, MYL4, TNNT3, $M Y M K, M Y B P C 2$ and TNNC2 (cluster black and pink) paralleled by expression of genes related to myotube maturation (TTN, MYF6 and MYL3; cluster blue; Figure 14). In summary, the trajectories of characteristic signature genes expression and GO term analysis allowed to annotate biological processes to 15 of 22 clusters (Table 5).

Table 5. Annotation of biological processes to coexpression clusters

\begin{tabular}{|c|c|}
\hline Cluster identifier & Biological process \\
\hline Black & Myotube development \\
\hline Blue & Myotube maturation \\
\hline Brown & Presomitic progenitor development \\
\hline Cyan & Not annotated \\
\hline Darkgreen & Cellular respiration \\
\hline Darkred & Myoblast development \\
\hline Green & Cilium organization \\
\hline Greenyellow & Not annotated \\
\hline Grey & Primitive streak development \\
\hline Lightcyan & DNA organization \\
\hline Lightgreen & Unfolded protein response \\
\hline Lightyellow & Not annotated \\
\hline Magenta & Not annotated \\
\hline Midnightblue & Not annotated \\
\hline Pink & Myotube development \\
\hline Purple & Not annotated \\
\hline Red & Presomitic progenitor development \\
\hline Royalblue & Lipid storage \\
\hline Salmon & Dermomyotome development \\
\hline Tan & Not annotated \\
\hline Turquoise & Presomitic progenitor development \\
\hline Yellow & Pluripotency \\
\hline
\end{tabular}


We next asked if any of the identified coexpression clusters overlap with developmentally regulated genes in human embryonic muscle. We made use of a published data set (GSE908776) containing the transcriptomes of embryonic presomitic mesoderm (PSM), nascent somite (SM), and developed somite (SM dev; (Xi et al. 2017)). Interestingly, several clusters (black, blue, royalblue, salmon, green, and pink) showed significant overlap with embryonic development (Figure 15). Please note that the very early developmental gene clusters (i.e presomitic mesoderm stage and earlier) are not represented in the embryo data set and therefore not overlapping.

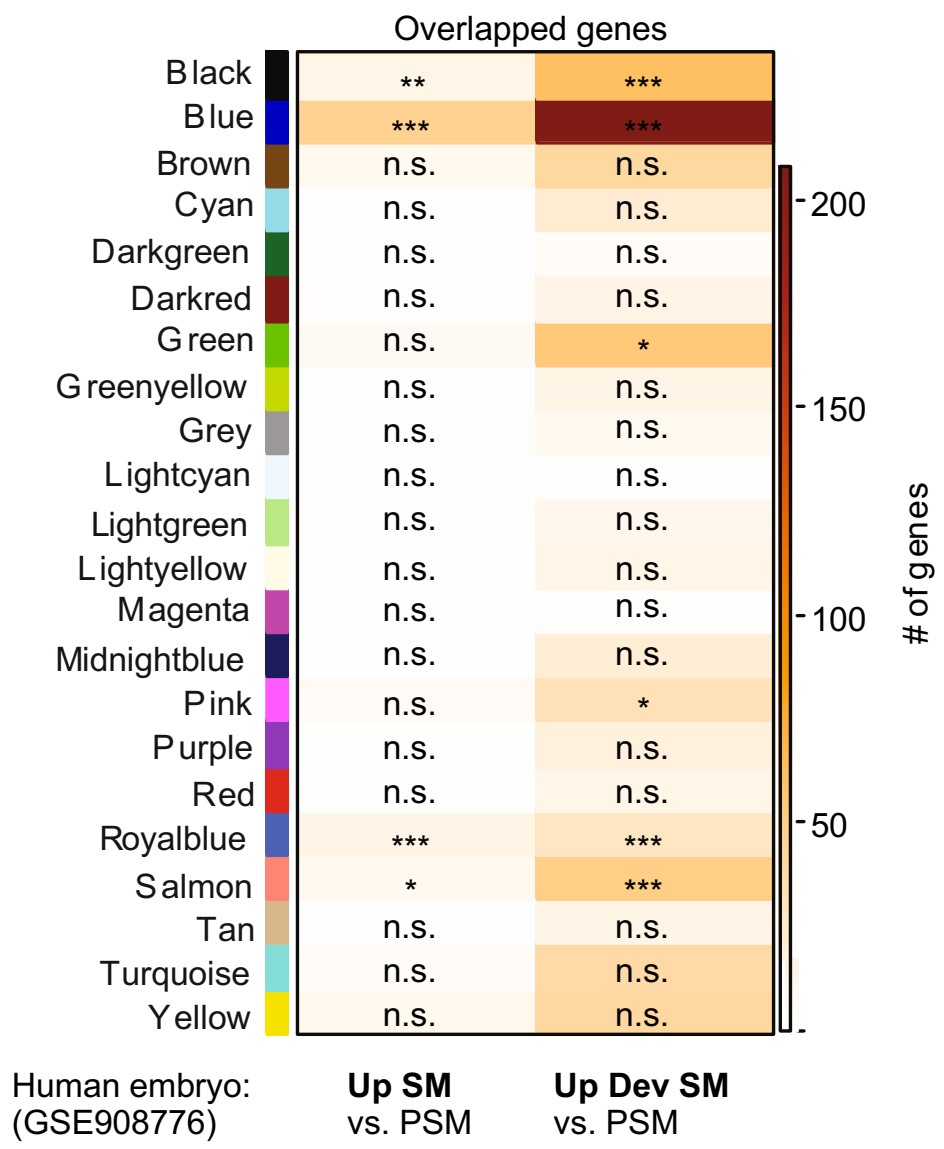

Figure 15. Coexpression clusters in vitro overlap with embryonic muscle development in vivo.

Developmentally regulated genes were identified based on a published human embryonic muscle data set (Xi et al. 2017). The table indicates the overlap of coexpression clusters genes to genes regulated between presomitic mesoderm (PSM) and nascent somite (SM) or presomitic mesoderm (PSM) to developed somite (Dev SM). Overlap is graded as either not significant (n.s.), $\mathrm{p}<0.05(*), \mathrm{p}<0.01(* *)$, or $\mathrm{p}<0.001(* * *)$. The color codes for the number of genes overlapping. Data obtained in collaboration with Dr. R. Islam, Fischer lab, Department for Epigenetics and Systems Medicine in Neurodegenerative Diseases. 
Next the co-expression analysis was utilized to identify transcriptome patterns coinciding with dissected phases of muscle development. We focused on the blue cluster as this showed the highest overlap with the developed somite in vivo and may therefore contain transcripts that are indicative of muscle maturation. Interestingly, cluster blue was highly enriched in signaling transcripts (Figure 16A). Among them several transcripts were identified that have been associated with muscle maturation such as NRG1 (Selvaraj et al. 2019), IGF1, VEGF (Xu et al. 2019) and thyroid hormone (Butler-Browne, Barbet, and Thornell 1990; Simonides and van Hardeveld 2008) (Figure 16B). Collectively, the transcriptome data indicates that hPSC differentiation in vitro is very similar to the developmental process in vivo and allows to dissect genes involved in particular stages of muscle development. Interestingly, also genes implicated in neuron development were found (Figure 16A), which prompted us to dissect the cell composition during skeletal muscle differentiation on a single cell level. 
A

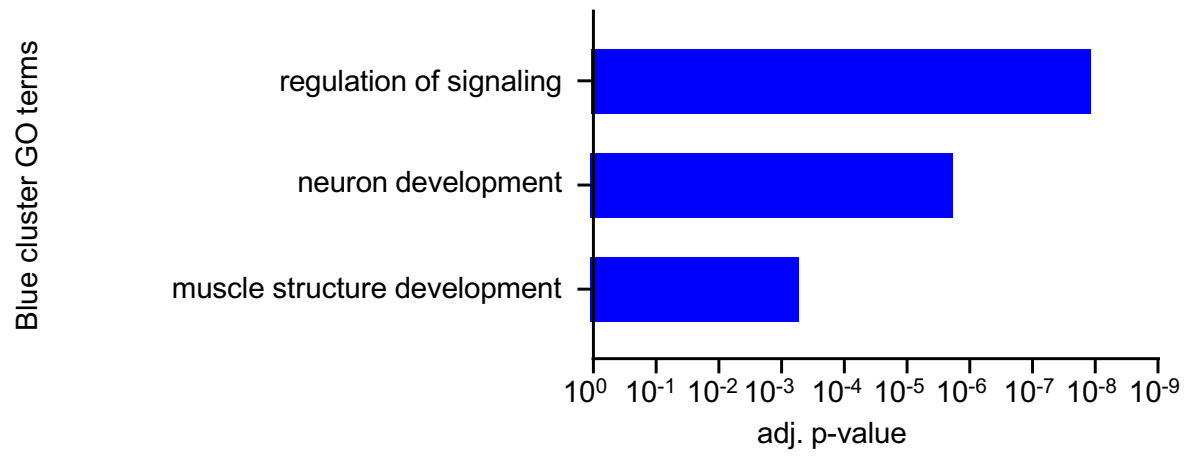

B

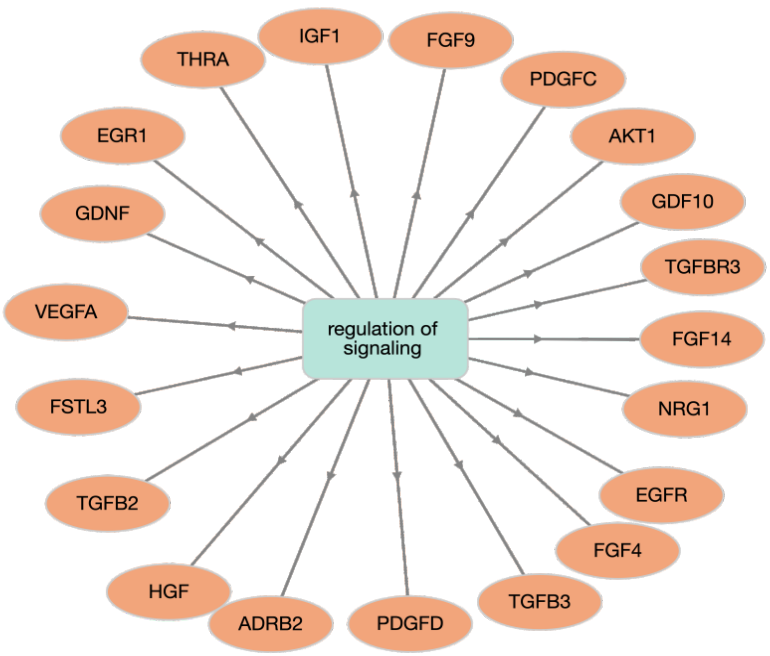

Figure 16. Identification of skeletal muscle maturation genes in coexpression cluster.

A, GO terms specifically enriched in coexpression cluster blue. B, List of genes associated with "regulation of signaling" in coexpression cluster blue.

\subsubsection{Composition of skeletal myocyte cultures on single cell level}

To gain further insight into the cell populations during skeletal muscle differentiation we investigated day 22 cultures by single nuclei sequencing (Kim et al. 2017). 8 cell populations were separated by unsupervised clustering (Figure 17A). To identify myogenic cells a panel of genes (muscle genes, Appendix) was extracted. This panel identified 3 myogenic cell populations accounting for $45 \%$ of the total cell population (Figure 17B, C). Non-muscle cells were identified 
as mesenchymal progenitor cells (41\%), neuroectodermal progenitor cells (9\%), and neurons (5\%) (Figure 17C). The composition of myogenic cells could be separated into $P A X 7+$ progenitors and more matured $M Y H 3+$ myoblasts. Consistent with the data from bulk RNA sequencing we found a population of $D C X+/ M A P T+$ neurons and $S O X 2+/ P A X 3+/ P A X 7+$ neuroectodermal progenitor cells. The mesenchymal cells showed an expression pattern consistent with fibro-adipogenic progenitor cells with combined expression of PDGFRA and brown fat transcription factor EBF2 (Rajakumari et al. 2013; Uezumi et al. 2010; Xi et al. 2020). These data indicate that the differentiation protocol not only recapitulates the embryonic development of muscle cells but also of the organotypic, supporting non-muscle cells. Next, we asked if these cell populations would generate an organotypic 3D muscle in vitro.

A

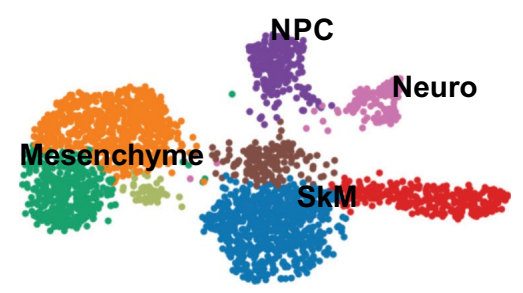

$\mathrm{D}$

Muscle:
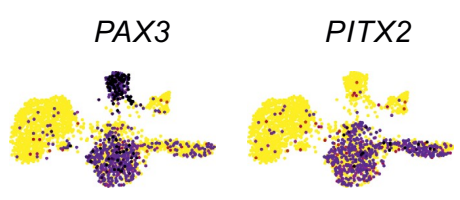

PAX7

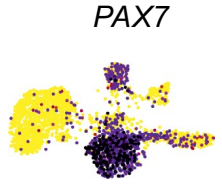

B

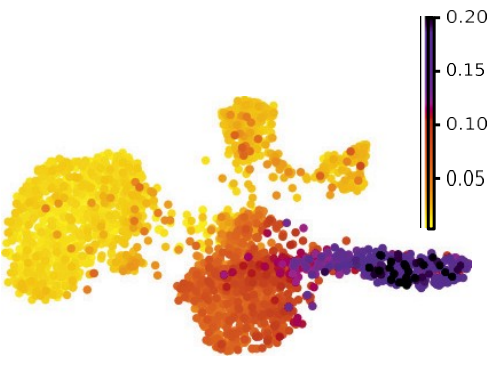

Non-muscle:

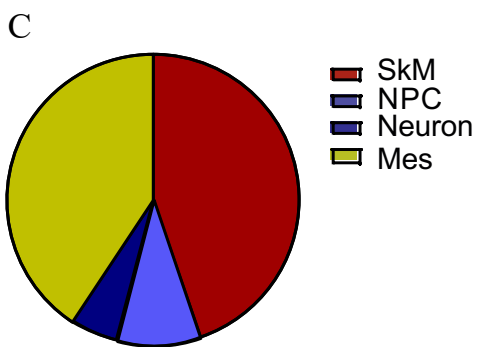

Total $=2671$

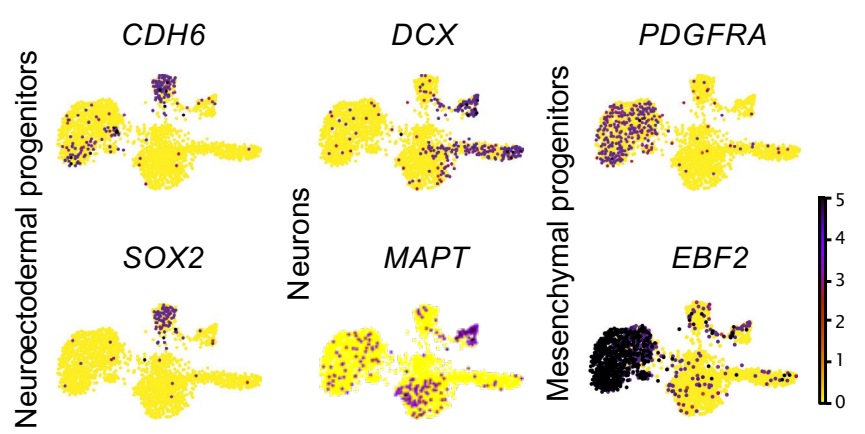

Figure 17. Single cell transcriptomes of skeletal muscle differentiation.

A, Unsupervised clustering (UMAP) of a day 22 skeletal muscle culture. B, A muscle gene panel identifies the myogenic cell clusters (skeletal muscle cells, SkM). C, Quantification of skeletal muscle cells (SkM), neuroectodermal progenitor cells (NPC), neurons, and mesenchymal progenitor cells (Mes). D, Expression levels of representative muscle-related genes and non-muscle genes. $C D H 15$ : Cadherin 15; DCX: Doublecortin; EBF2: EBF transcription factor 2; MAPT: Microtubule associated protein tau; MYH3: Myosin heavy chain 3; NEB: Nebulin; PAX3: Paired box gene 3; PAX7: Paired box gene 7; PDGFRA: Platelet derived growth factor receptor alpha; PITX2: Paired like homeodomain 2; SOX2: SRY-box transcription factor 2. Data obtained in collaboration with S. Sadman and D. Krüger, Fischer lab, Department for Epigenetics and Systems Medicine in Neurodegenerative Diseases. 


\subsection{Human engineered skeletal muscle}

\subsubsection{Generation of human bioengineered skeletal muscle organoids from iPSC}

To generate human skeletal muscle in vitro it was first tested if the established in vitro myogenesis protocol is capable of directing iPSCs into skeletal muscle tissue in a 3D environment. In contrast to monolayer 2D culture iPSCs were embedded in a hydrogel containing collagen type 1 and Matrigel $^{\mathrm{TM}}$ and then subjected to skeletal muscle differentiation (Figure 18A). The mixture was cast into circular molds to consolidate into mechanically stable ring-shaped tissues which were maintained under isometric mechanical load in maturation medium for a minimum of 4 weeks.

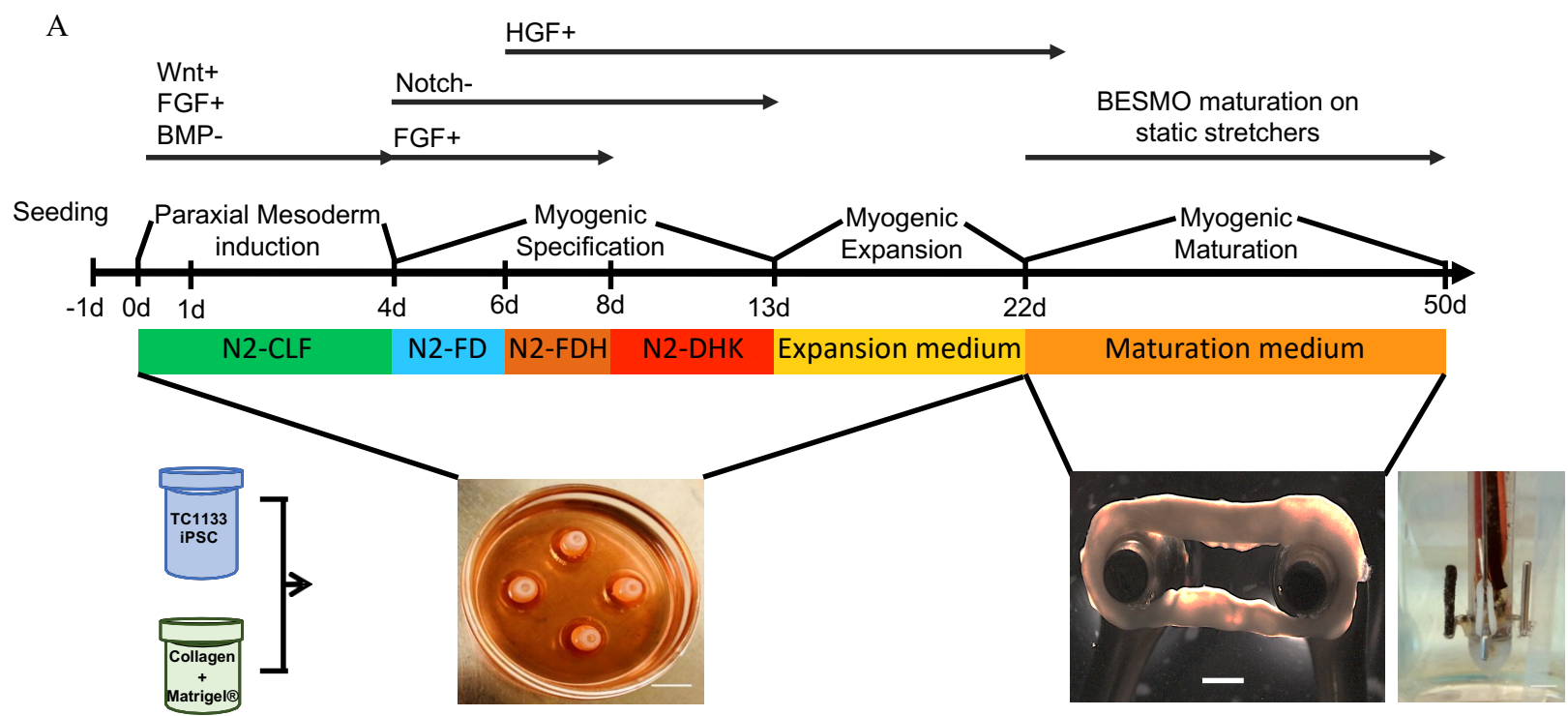

B

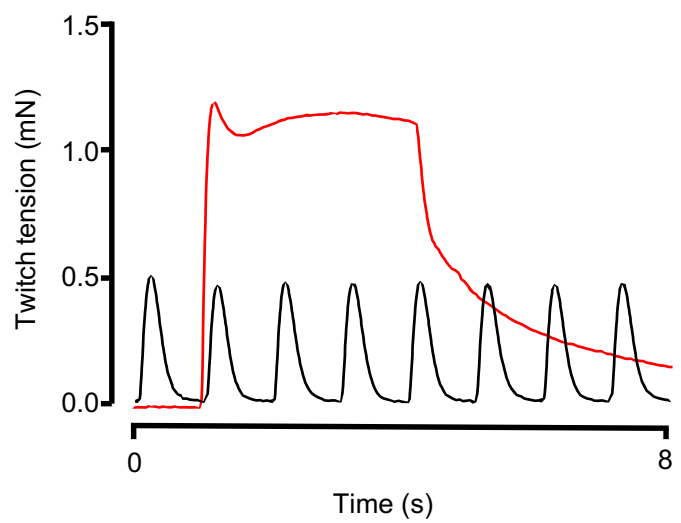

C

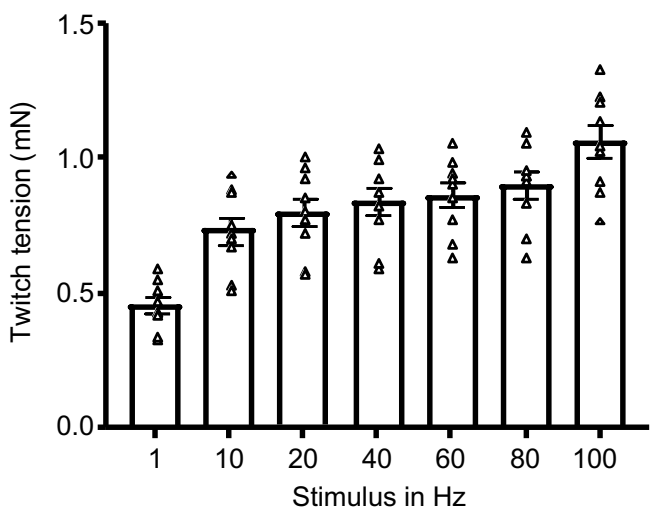

Figure 18. Generation of functional human bioengineered skeletal muscle (BSM) organoid from PSCs. A, Bioengineered skeletal muscle (BSM) organoids were generated from TC1133 (iPSC 1) iPSC mixed with collagen type 1 and Matrigel ${ }^{\mathrm{TM}}$ in a ring-shaped hydrogel. After consolidation in PDMS casting molds, BSMs were directed 
towards skeletal muscle using the protocol established in monolayer cultures. +/- indicates pathway activation/inhibition, respectively. Following functional maturation under isometric load up to 4 wks (day 22 to 50), twitch tension (TT) was measured under isometric conditions in a thermostatted organ bath. Scale bar: $1 \mathrm{~mm}$. B, Representative force traces of 4 wks old BSM with electrical stimulation at $1 \mathrm{~Hz}$ (black curve) and at $100 \mathrm{~Hz}$ (red curve). C, Quantification of the twitch tension (TT) generated by 4 wks old BSM in response to increasing stimulation frequencies; $n=9$ /group.

In accordance with the results from monolayer differentiation a muscle tissue - termed bioengineered skeletal muscle (BSM) organoid - was generated with characteristic skeletal muscle function. Without electrical stimulation BSM demonstrated slow, spontaneous, non-coherent contractions. At $1 \mathrm{~Hz}$ electrical stimulation BSM generated single twitches whereas at higher frequencies tetanic contractions were observed which were maximal at $100 \mathrm{~Hz}(1.1 \pm 0.1 \mathrm{mN}$; Figure 18B,C).

To investigate the muscle content of the BSM immunostaining of ACTIN and dystrophinassociated glycoprotein, $\beta$-DYSTROGLYCAN was performed, which indicated that $26 \%$ of the total cross sectional area contained muscle cells (Figure 19A). Muscle cells were nicely aligned and cross striated indicating a well developed sarcomeric structure in agreement with the robust contractile function. In line with the transcriptome data of monolayer cultures, BSM also contained mature neurofilaments positive for the motor neuron marker SMI32 (Figure 19B). 
A
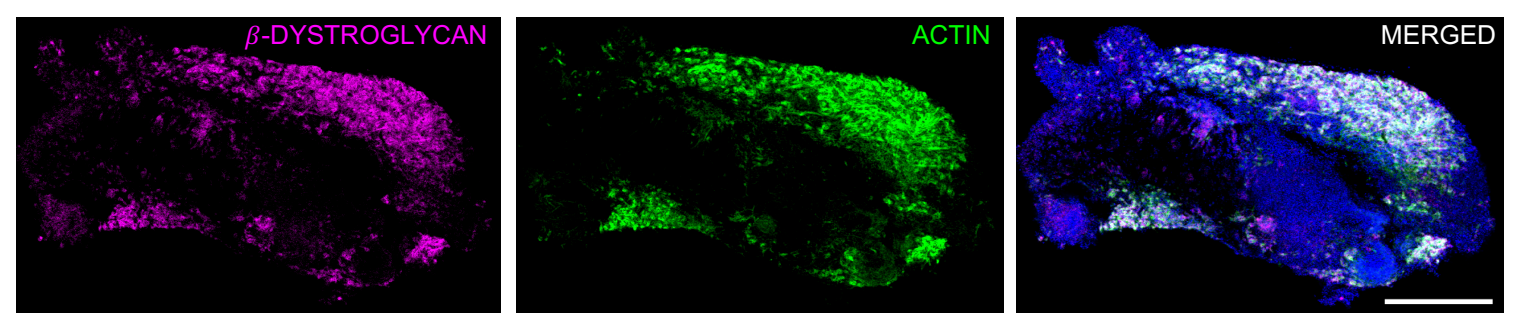

B
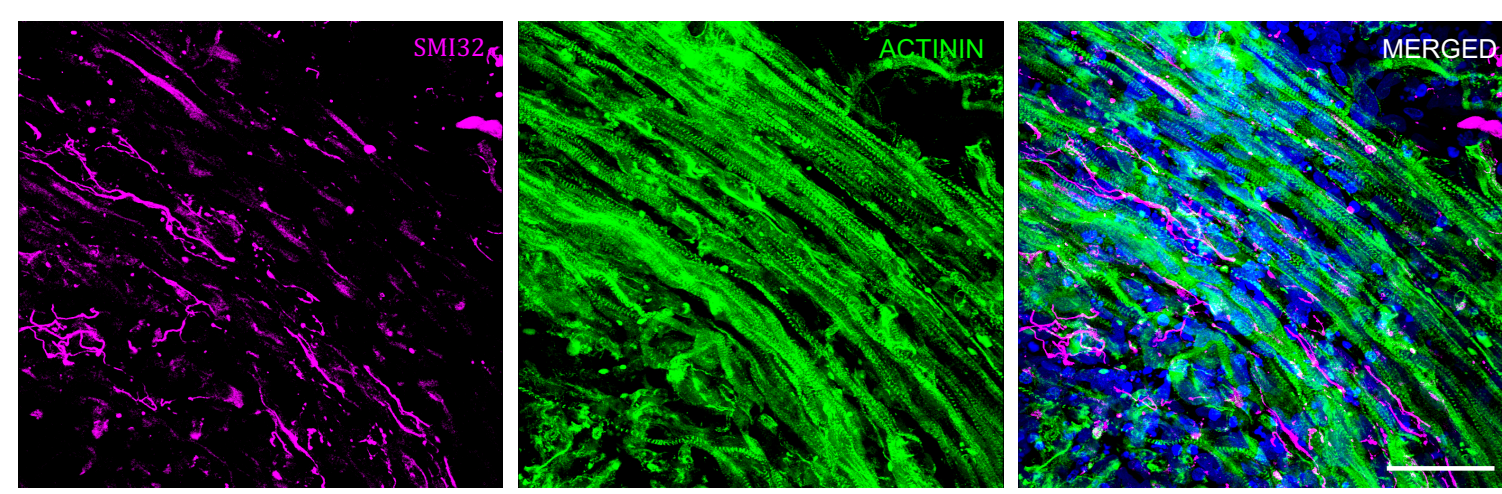

Figure 19. Generation of highly organized skeletal myofibrils in human bioengineered skeletal muscle. A, Immunostaining of the total muscle area in cross sections of BSM with $\beta$-DYSTROGLYCAN (magenta), ACTIN+ (green), and Nuclei (blue). B, Immunostaining of neurofilament heavy SMI32 (magenta), sarcomeric $\alpha$-ACTININ (green) and Nuclei (blue) in longitudinal sections of BEM. Scale bar: $500 \mu \mathrm{m}$ (A) and $50 \mu \mathrm{m}$ (B).

\subsubsection{Generation of human engineered skeletal muscle with organotypic function}

In an alternative approach to generate in vitro skeletal muscle with increased muscle mass and function we aimed to control the cellular input of the 3D model. Skeletal muscle cultures including PAX7+/MYOD+ myogenic progenitors and primary myotubes on day 22 were found to be the optimal for ESM generation as cultures could be readily dissociated. Older cultures tended to be too dense with a lot of extracellular matrix (ECM) that prohibited efficient enzymatic digestion and inadvertently caused excessive cell death. In addition, spontaneous twitches of skeletal myocytes caused the 22+ day old differentiated culture to detach as a sheet of cells from the substrate. Dissociated cultures were reassembled into engineered skeletal muscle (ESM) by casting them into a collagen-1/Matrigel hydrogel. After consolidation for 1 week in expansion medium the ESM was maintained under isometric mechanical load in maturation medium for 4 to 8 weeks (Figure 20A).

During mechanical loading, spontaneous, non-coherent twitches were observed in ESM. By 4 weeks maturation ( 5 weeks old) ESM robustly developed measurable force with typical properties 
of skeletal muscle such as positive force-frequency response and tetanic contractions with increasing stimulation frequencies (Figure 20 B,C).

By prolonging the culture for up to 9 weeks under defined serum-free conditions, tetanus twitch tension generated by ESM at $100 \mathrm{~Hz}$ stimulation frequency, significantly increased form $2.9 \pm 0.3$ $\mathrm{mN}(\mathrm{n}=8)$ at 5 weeks to $5.5 \pm 0.3 \mathrm{mN}(\mathrm{n}=4)$ at 9 weeks (Figure 20B, C) which may indicate advanced sarcomere alignment in parallel with increased myofibril density (Racca et al. 2013). 
A

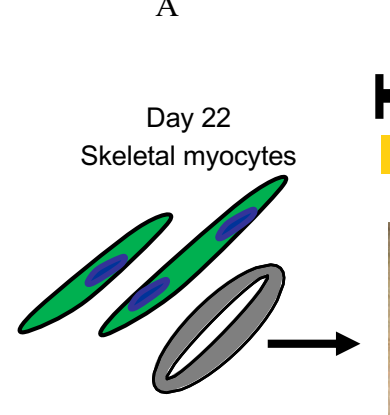

Collagen \& Matrigel

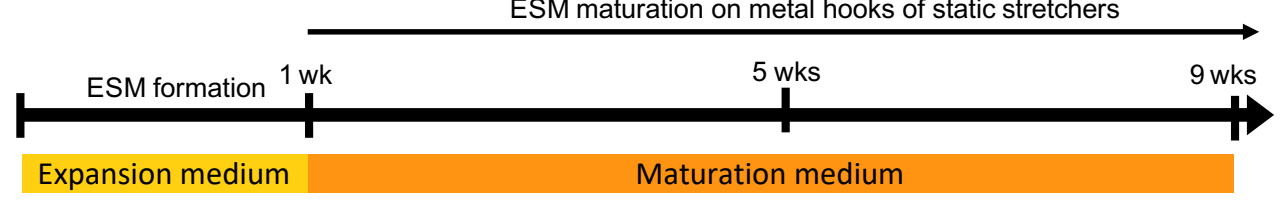

B
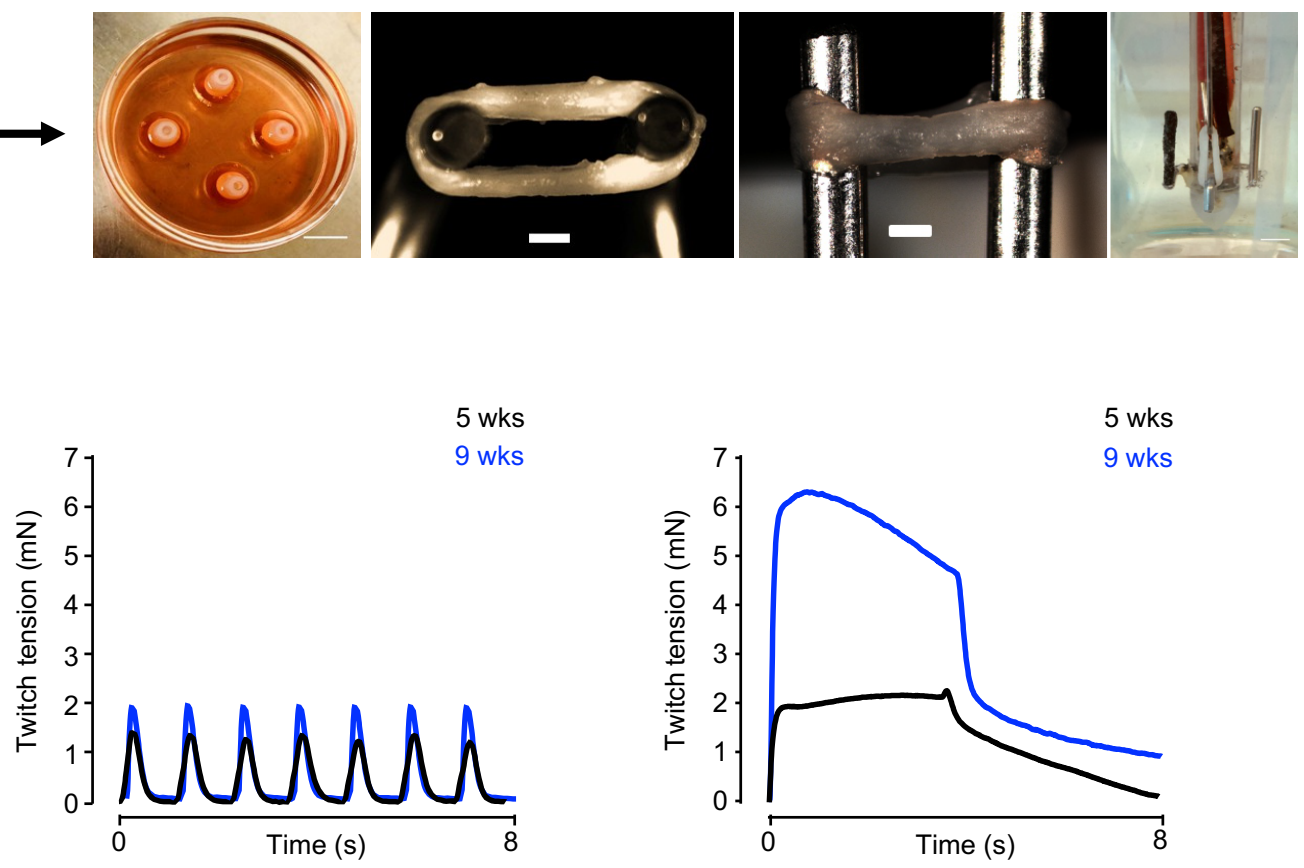

$\mathrm{C}$

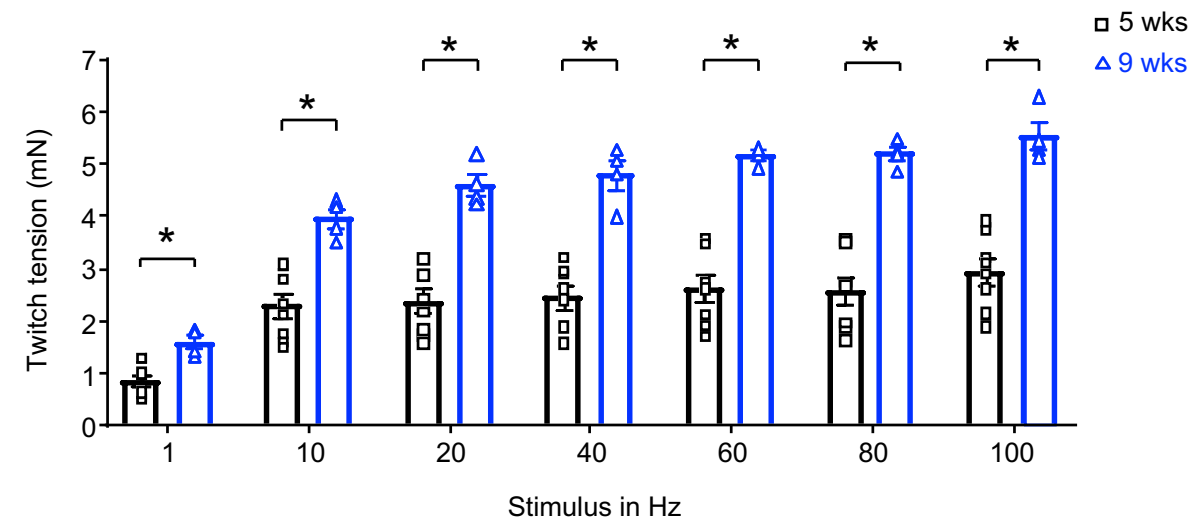

Figure 20. Contractile function of engineered skeletal muscle increases with time.

A, Scheme of engineered skeletal muscle (ESM) generation from human PSC-derived skeletal muscle populations including PAX7+/MYOD+ myogenic progenitors and primary myotubes mixed with collagen type 1 and Matrigel ${ }^{\mathrm{TM}}$ in a ring-shaped hydrogel. ESM formation in expansion medium for 1 week in PDMS casting molds, functional maturation under isometric mechanical load (ESM on metal hooks of the static stretcher) for up to 9 wks; measurement of twitch tension under isometric conditions in a thermostatted organ bath. Scale bar: $1 \mathrm{~mm}$. B, Representative traces of single twitches of $5 \mathrm{wks}$ old and $9 \mathrm{wks}$ old ESM at $1 \mathrm{~Hz}$ (left panel) and at $100 \mathrm{~Hz}$ (right panel) stimulation frequencies. $\mathbf{C}$, Twitch tension of $5 \mathrm{wks}$ and $9 \mathrm{wks}$ old ESM in response to increasing stimulation frequencies; $\mathrm{n}=$ 8 /group at 5 wks and $n=4$ /group at 9 wks, ${ }^{*} p<0.05$ by 2 -way ANOVA and Tukey's test. 


\subsubsection{Advanced muscle structure of engineered skeletal muscle}

To characterize the skeletal muscle morphology immunostaining studies were performed on 9 weeks ESM. Analysis of ESM cross sections showed densely packed muscle cells embedded in a Laminin + extracellular matrix making up $68 \%$ of the total cross sectional area (Figure 21A). High power images of ESM cross sections further demonstrated a high resemblance to skeletal muscle with compact cylindrical myofibers and localized, membrane-associated organization of the dystrophin-associated glycoprotein, $\beta$-DYSTROGLYCAN (Figure 21B).

A

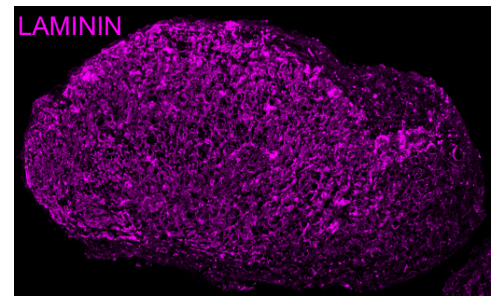

B

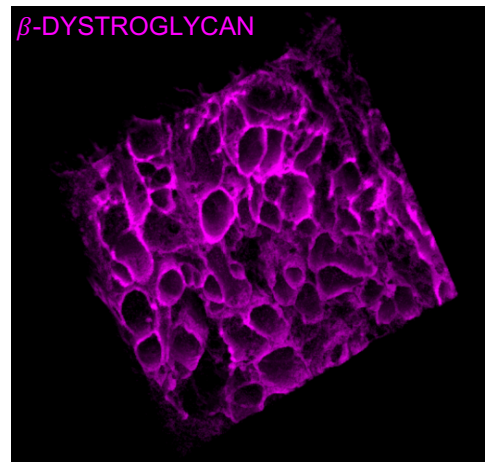

$\mathrm{C}$

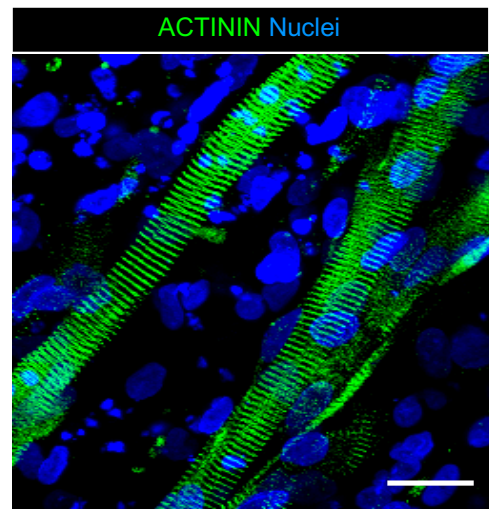

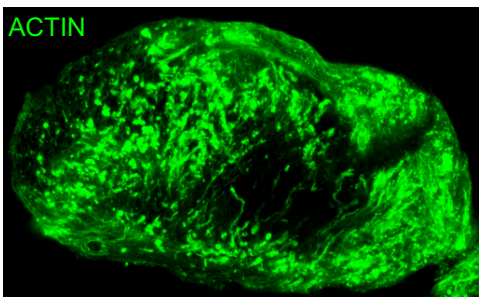

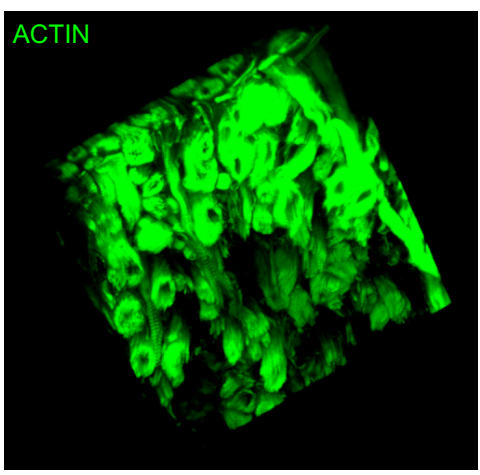

$\mathrm{D}$

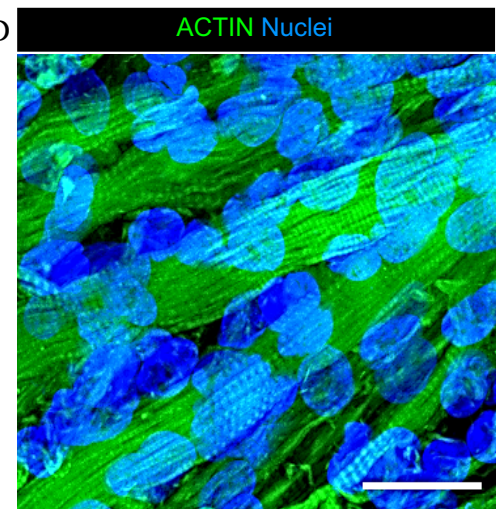

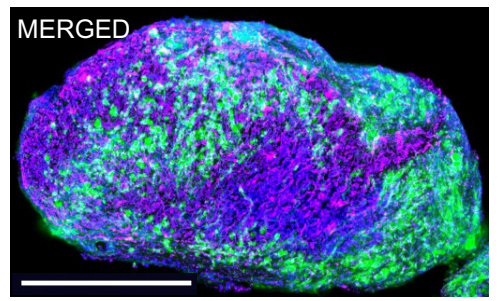

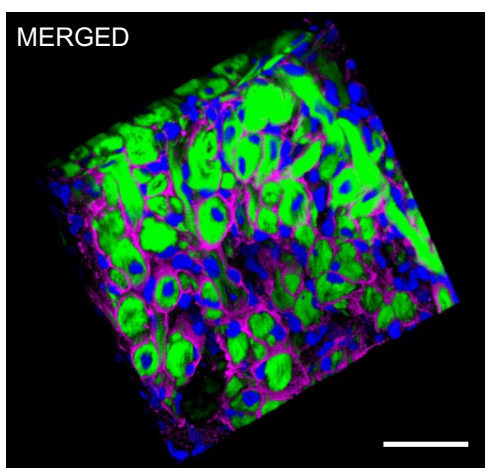

$\mathrm{E}$

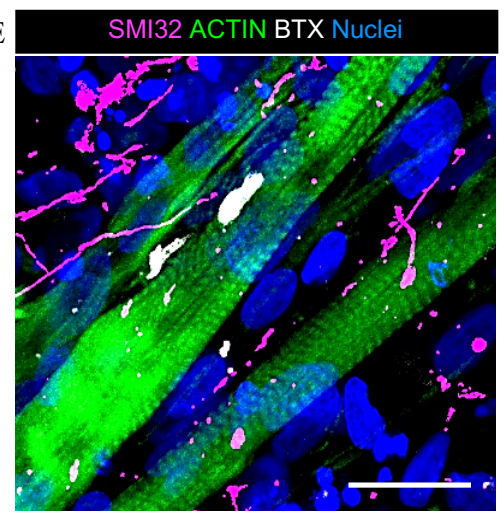

Figure 21. Advanced development of skeletal muscle structures in human engineered skeletal muscle.

A, Immunostaining of ACTIN+ muscle cells (green) and LAMININ+ extracellular matrix (magenta) in a representative cross section of 9 wks old ESM. B, Immunostaining of $\beta$-DYSTROGLYCAN (magenta) in the sarcolemma of ACTIN+ muscle fibers (green) in a cross section of ESM. C, D and E, Immunostaining of striated muscle fibers in longitudinal sections of ESM for $\mathbf{C}$, sarcomeric $\alpha$-ACTININ (green) and Nuclei (blue), D, ACTIN (green) and Nuclei (blue), E, ACTIN (green), neurofilament heavy SMI32 (magenta), $\alpha$-bungarotoxin (BTX) labeled nicotinic acetylcholine receptors (white) and Nuclei (blue). Scale bar: $500 \mu \mathrm{m}$ (A), $40 \mu \mathrm{m}$ (B) and $20 \mu \mathrm{m}$ (C,D,E). 
Longitudinal sections of ESM further demonstrated advanced differentiated and aligned skeletal myofibrils with multiple nuclei and regular arrangement of sarcomeres labeled by sarcomeric $\alpha-$ ACTININ and ACTIN (Figure 21C,D). The average muscle fiber diameter was $12 \pm 0.21 \mu \mathrm{m}$ at 9 weeks of maturation.

Clusters of $\alpha$ - bungarotoxin positive nicotinic acetylcholine receptors (AChR) were found on the periphery of skeletal muscle fibers (Figure 21E). Interestingly, and in line with the transcriptome data the presence of neurons in ESM was confirmed by SMI32 as a specific marker of mature neurofilaments in motor neurons. Of note, some of these were seemingly innervating muscle cells by close association of the motor neuron's axonal terminals with the $\alpha$-bungarotoxin-positive motor end plate (Figure 21E). To test the functionality of the nicotinic acetylcholine receptors the unspecific cholinergic receptor agonist carbachol $(1 \mu \mathrm{mol} / \mathrm{L})$ was applied. Incubation of ESM with carbachol lead to a rapid decrease in stimulated contractions indicative of a depolarizing muscle block. This effect was completely reversible after washing out the carbachol (Figure 22).
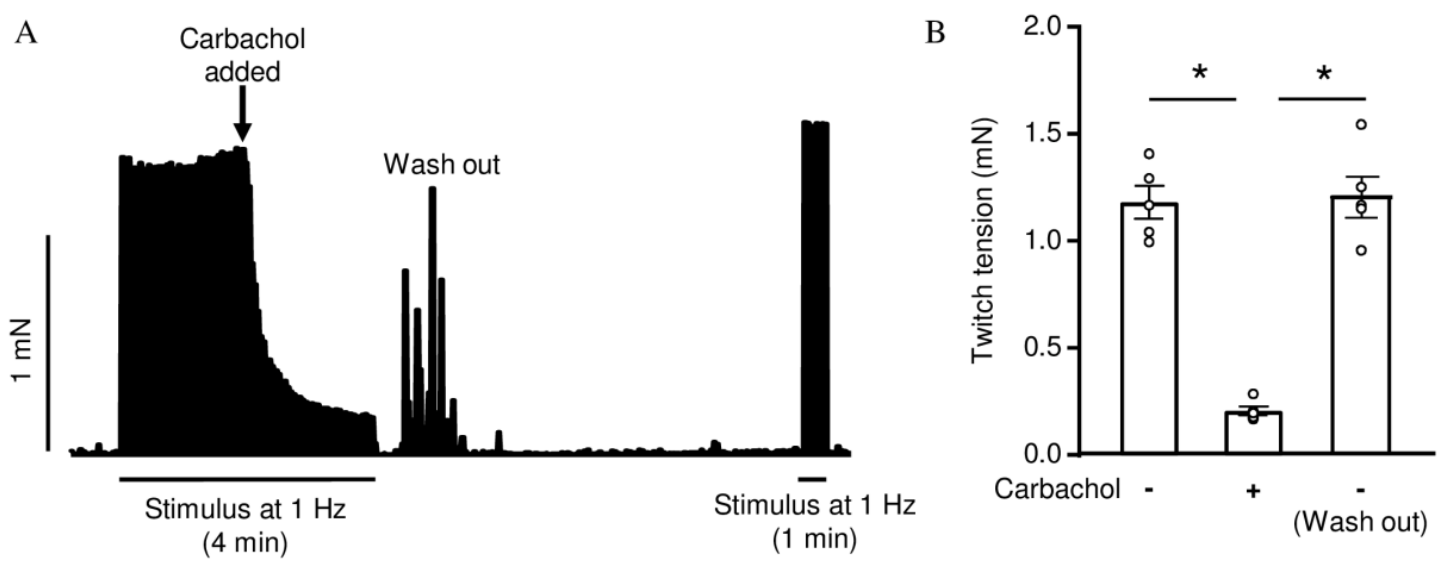

Figure 22. Functional nicotinic acetylcholine receptors in ESM.

A, Representative recording of an ESM twitch tension (bar indicates $1 \mathrm{mN}$ ) at $1 \mathrm{~Hz}$ electrical stimulation. The unspecific cholinergic receptor agonist carbachol $(1 \mu \mathrm{M})$ is added where indicated and later washed out from the organ bath. B, Quantification of twitch tension at $1 \mathrm{~Hz}$ stimulation before and after treatment of ESM with $1 \mu \mathrm{M}$ carbachol and after washout; $\mathrm{n}=5 ;{ }^{*} \mathrm{p}<0.05$ by 1 -way ANOVA and Tukey's multiple comparison test.

Additional ultrastructural analyses confirmed a high skeletal muscle mass with advanced stages of myofibrillogenesis. Organized sarcomeres with distinct banding pattern including I- bands, Abands, $\mathrm{M}$-lines and $\mathrm{Z}$ disks were observed (Figure 23A). Average length of sarcomere in ESM was $1.93 \pm 0.06 \mu \mathrm{m}$. Membranous structures of the triad, composed of a central T-tubule surrounded 
by two terminal cisternae from the sarcoplasmic reticulum were identified (Figure 23B) (AlQusairi and Laporte 2011). Peripheral nuclei and well-differentiated mitochondria with dense matrix and developed cristae were found aligned with compact sarcomeres (Figure 23C, D).
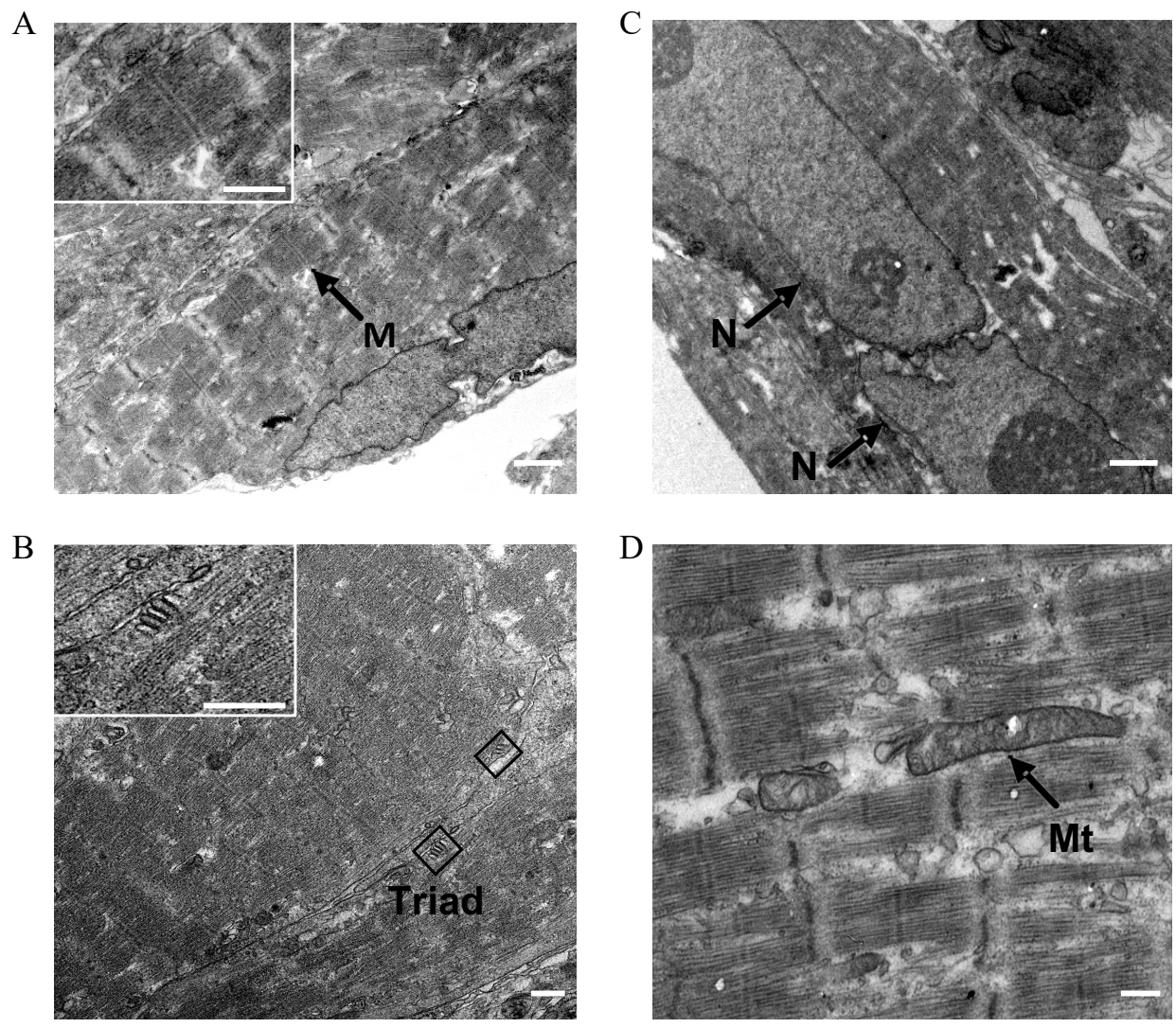

Figure 23. Ultrastructure of human engineered skeletal muscle.

Transmission electron microscopy (TEM) images of A: sarcomeric structure, B: Triad junction, C: a long multinucleated (2 nuclei) muscle cell and D: mitochondria along the muscle fibers in ESM. M: M-line, Mt: Mitochondria, N: nuclei. Scale bar: $1 \mu \mathrm{m}(\mathrm{A}), 500 \mathrm{~nm}(\mathrm{~B}), 1 \mu \mathrm{m}(\mathrm{C})$ and $250 \mathrm{~nm}(\mathrm{D})$.

\subsubsection{Creatine supplementation enhances force of ESM}

Although the twitch tension recorded for ESM exceeds previously published models it is still far from the force production of adult muscle (Racca et al. 2013). Therefore, the next aim was to further enhance the ESM functionality. It has been demonstrated that strength training in combination with creatine supplementation led to greater skeletal muscle mass, strength gain and fatigue resistance (Bonilla and Moreno 2015; Cooke et al. 2009; Olsen et al. 2006; Willoughby 
and Rosene 2001). Since loading the ESM on static stretchers is resembling isometric exercise for the tissue, we hypothesized that supplementation of maturation medium with creatine monohydrate would enhance the function of ESM.

Maturation medium of ESM was supplemented with $1 \mathrm{mM}$ creatine monohydrate during early maturation (5 weeks) or late maturation (9 weeks) time windows (Figure 24A). Creatine enhanced the twitch tension of ESM by $1.5 \pm 0.2$ fold at week 5 and by $1.3 \pm 0.02$ fold at week 9 (Figure 24B).

A

+Creatine monohydrate $(1 \mathrm{mM})$

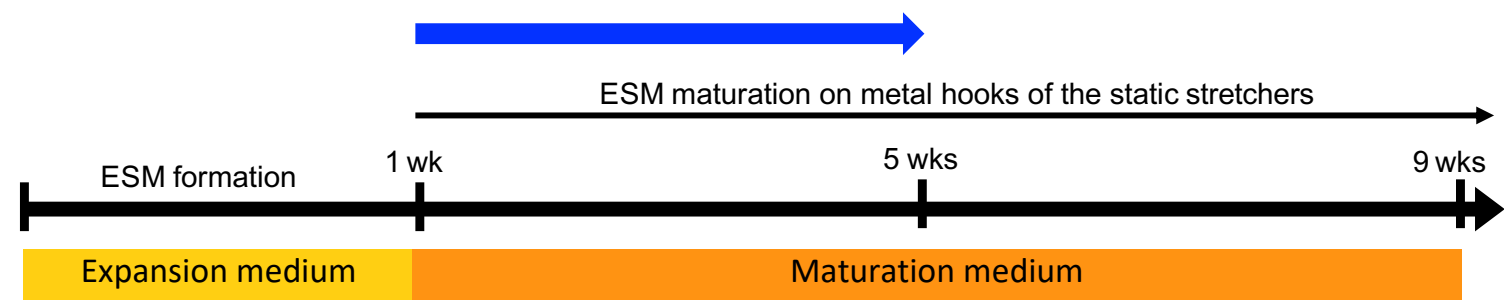

B

5 wks ESM

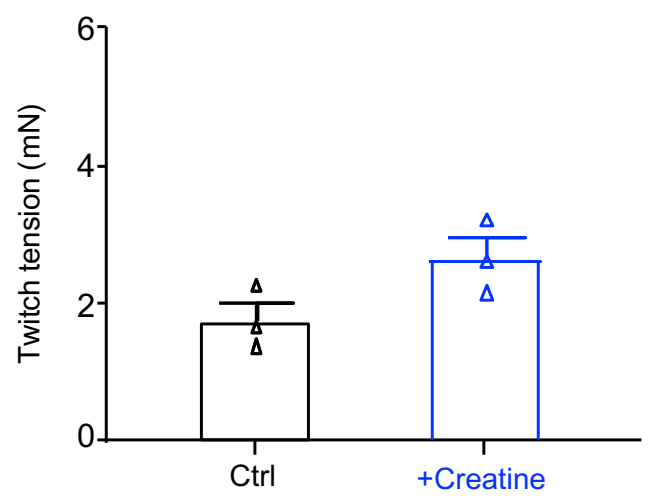

9 wks ESM

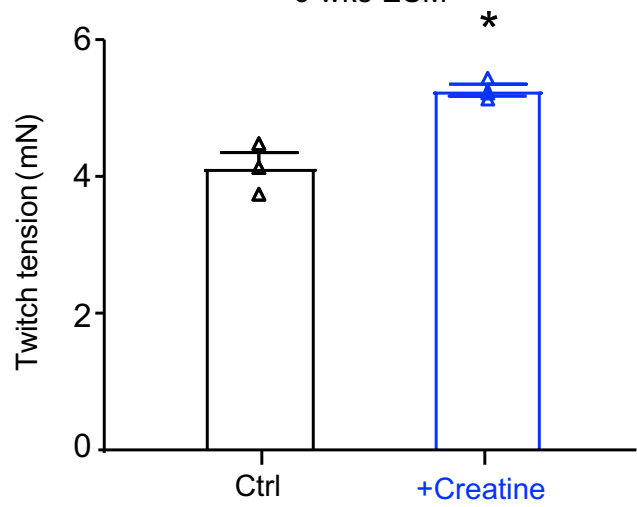

Figure 24. Creatine supplementation increases ESM twitch tension.

A, Scheme indicating the experimental design for ESM treatment with $1 \mathrm{mM}$ creatine monohydrate (green arrows) for 4 wks. B, Quantification of tetanic twitch tension at $100 \mathrm{~Hz}$ stimulation twitch tension of control ESM (Ctrl; black bars) vs. creatine treated ESM (+creatine, blue bars) at $5 \mathrm{wks}$ and 9 wks maturation time; $\mathrm{n}=3$ /group at each time point, ${ }^{*} \mathrm{p}<0.05$ by Student's t-test. 


\subsubsection{Maturation of myosin isoforms by $\mathrm{T} 3$ treatment}

Next, the knowledge gained from the transcriptome data was exploited to enhance the functionality of ESM. Thyroid hormone receptor was part of "maturation cluster" (coexpression cluster blue) which prompted us to test if ESM would respond to Triiodo-L-thyronine (T3) treatment. T3 does not increase maximal force production but has been described to enhance physiological maturation of skeletal muscle by triggering the transition in myosin heavy chain isoform expression towards adult fast myosin isoforms (Larsson et al. 1994; Schiaffino et al. 1988, 2015; Simonides and van Hardeveld 2008). It was therefore investigated if addition of T3 to early maturation (5 weeks) or late maturation (9 weeks) phase of ESM would influence ESM function (Figure 25A).

A
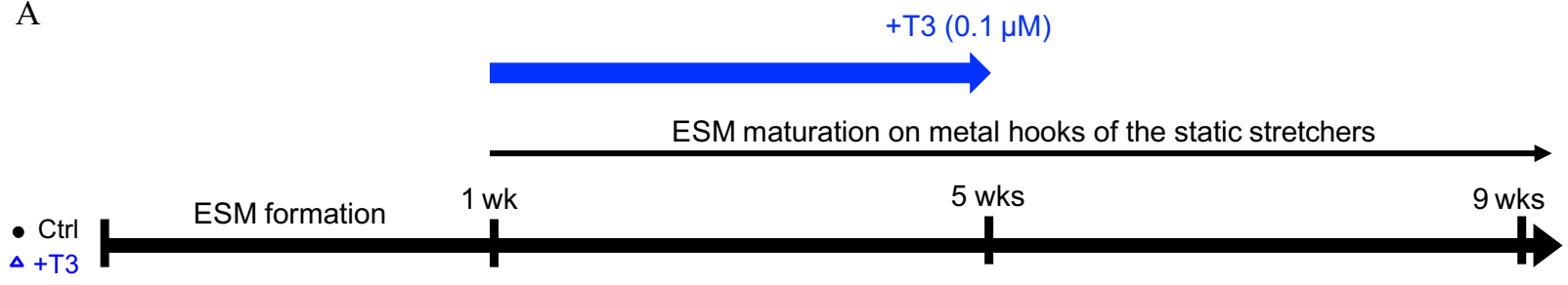

Expansion medium Maturation medium

B
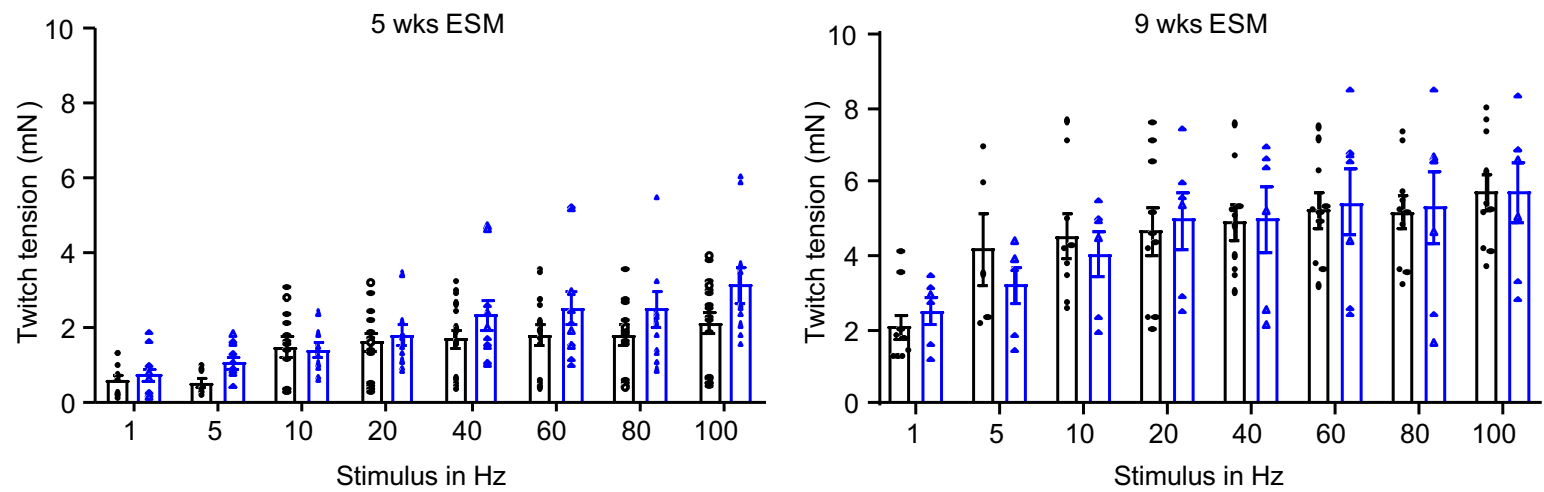

Figure 25. Effect of thyroid hormone on twitch tension generated by ESM.

A, Scheme of experimental design of ESM maturation for 5 or 9 wks with or without additional application of 0.1 $\mu \mathrm{mol} / \mathrm{L}$ Triiodo-L-thyronine (T3) for $4 \mathrm{wks}$. B, Twitch tension in response to increasing stimulation frequencies of 5 wks and 9 wks old ESM cultured with (blue bars) or without T3 (black bars); n=11-16/group at 5 wks; 7-10/group at 9 wks). 
T3 treatment did not influence the maximal twitch tension (Figure 25B), but clearly shortened the duration of single twitches of both 5 weeks and 9 weeks ESM. Accordingly, the speed of contraction (Time to $90 \%$ contraction -T1) of single twitches as well as relaxation (Time to 50\% relaxation -T2) at 5 weeks and 9 weeks was significantly decreased (Figure 26A,B). In addition, the rate of force development (RFD) in tetanic contractions ( $100 \mathrm{~Hz}$ stimulation frequency) tended to be faster in ESM treated with T3. The rate of force decline was enhanced in 5 weeks ESM treated with T3 with a tendency also in 9 weeks ESM (Figure 26C,D).

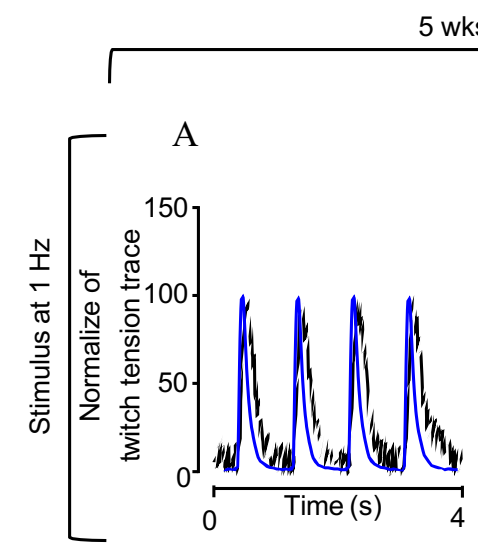

wks ESM
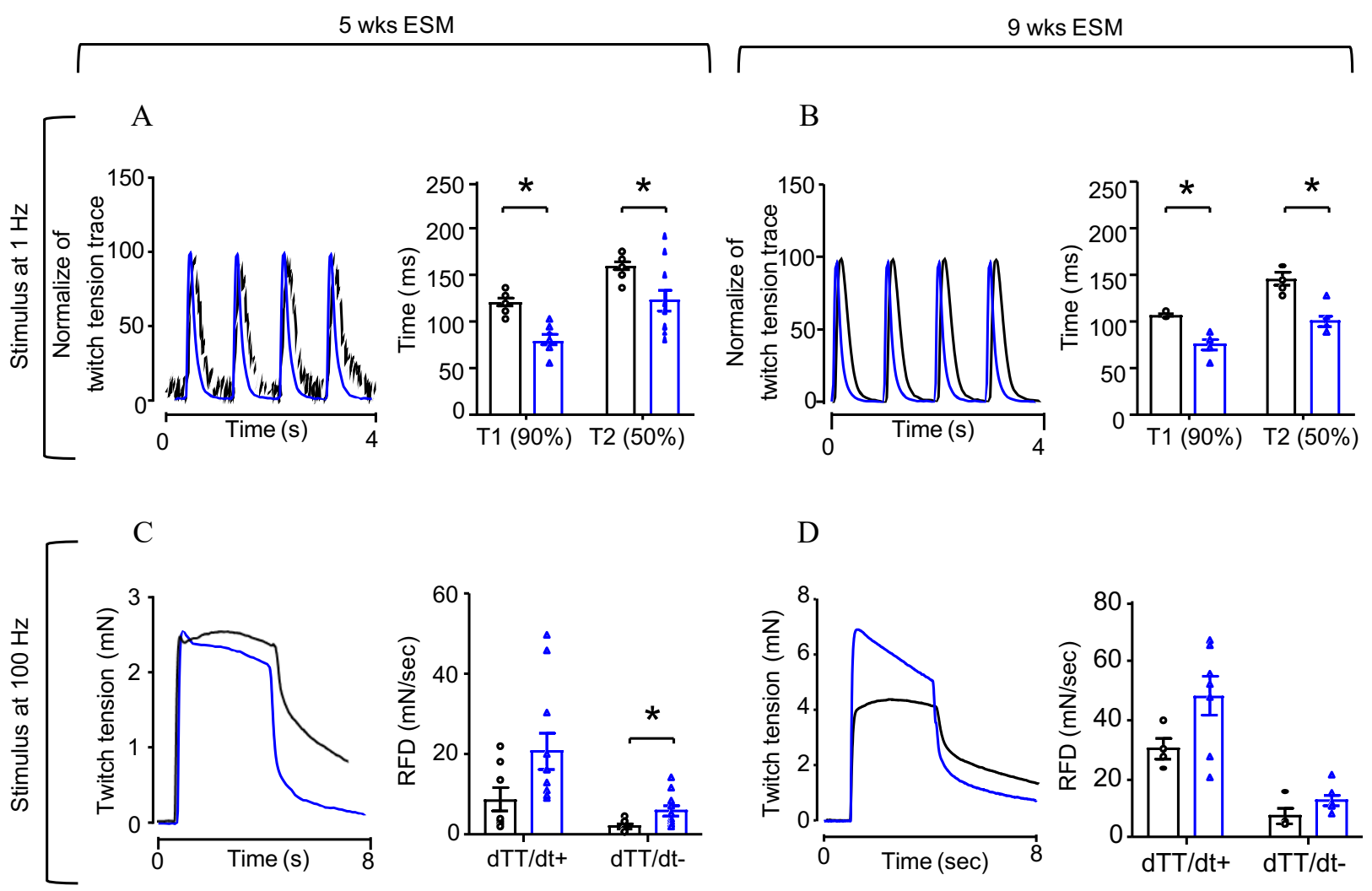

Figure 26. Advanced contractile kinetics of ESM by thyroid hormone treatment.

A, Normalized representative traces of single twitches of 5 wks old control (black line) or +T3 (blue line) ESM at 1 $\mathrm{Hz}$ (left panel); Quantification of contraction (T1) and relaxation (T2) time of single twitches of 5 wks old control (black bars) or $+\mathrm{T} 3$ (blue bars) ESM at $1 \mathrm{~Hz}$ (right panel); $\mathrm{n}=8-11$ /group, ${ }^{*} \mathrm{p}<0.05$ by Student's t-test. B, Normalized representative traces of single twitches of 9 wks old control (black line) or +T3 (blue line) ESM at $1 \mathrm{~Hz}$ (left panel); Quantification of contraction (T1) and relaxation (T2) time of single twitches of 9 wks old control (black bars) or $+\mathrm{T} 3$ (blue bars) ESM at $1 \mathrm{~Hz}$ (right panel); $\mathrm{n}=5-6$ /group, ${ }^{*} \mathrm{p}<0.05$ by Student's t-test. C, Representative traces of twitch tension of 5 wks old control (black line) or +T3 (blue line) ESM at $100 \mathrm{~Hz}$ tetanus (left panel); Rate of force development (RFD; rate of contraction: $\mathrm{dTT} / \mathrm{dt}+$ and rate of relaxation: dTT/dt-) of 5 wks old control (black bars) or +T3 (blue bars) ESM at $100 \mathrm{~Hz}$ tetanus (right panel); $\mathrm{n}=8-11$ /group, ${ }^{*} \mathrm{p}<0.05$ by Student's t-test. D, Representative traces of twitch tension of 9 wks old control (black line) or +T3 (blue line) ESM at $100 \mathrm{~Hz}$ tetanus (left panel); Rate of force development (RFD; rate of contraction: dTT/dt+ and rate of relaxation: dTT/dt-) of 9 wks old control (black bars) or +T3 (blue bars) ESM at $100 \mathrm{~Hz}$ tetanus (right panel); $\mathrm{n}=4$-7/group. 
The tetanus threshold (i.e. frequency where single twitches fuse to tetani) is greater in mammalian adult fast muscle fiber in comparison to slow muscle fibers (Buller and Lewis 1965). The tetanus threshold of ESM with and without T3 treatment was analyzed by calculation of a fusion index (Figure 27A) derived from twitch recordings at increasing stimulation frequencies (Figure 27B). The tetanus fusion index was different in ESM treated with T3 with a significant shift towards higher stimulation frequencies (50\% fusion at $3.92 \pm 0.24 \mathrm{~Hz}$ vs $5.44 \pm 0.05 \mathrm{~Hz}$ in control ESM vs. $\mathrm{ESM}+\mathrm{T} 3$, respectively (Figure 27C). Collectively, these functional data suggest that T3 enhances fast muscle properties of ESM.

A

Control

T3
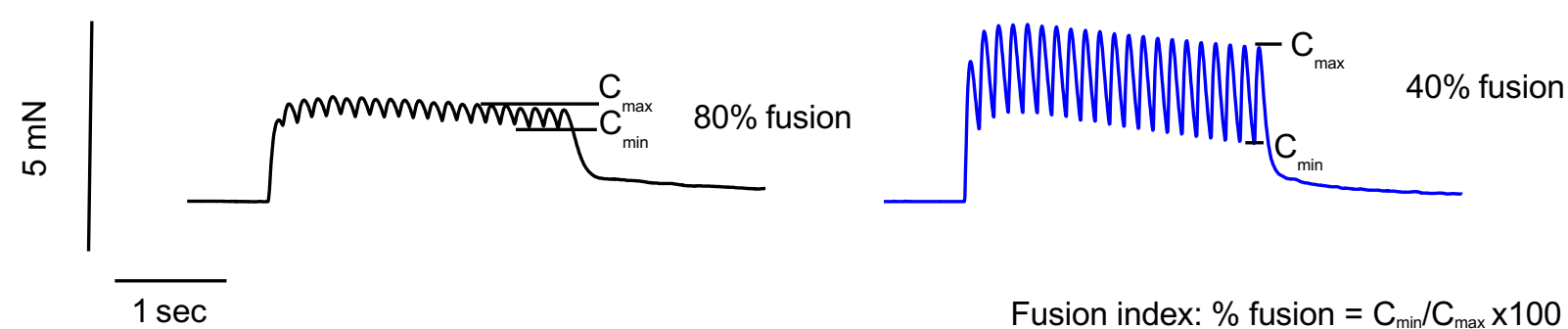

Fusion index: $\%$ fusion $=\mathrm{C}_{\min } / \mathrm{C}_{\max } \times 100$

B

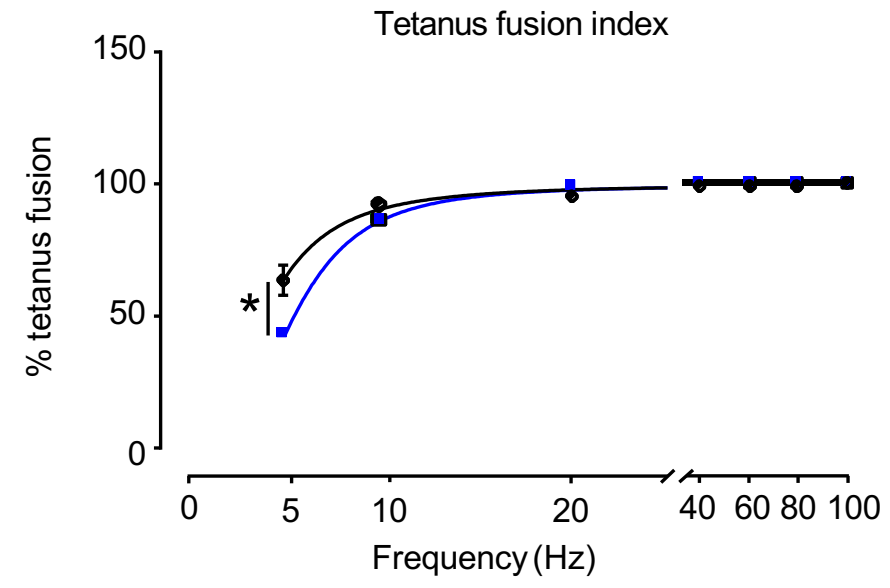

$\mathrm{C}$

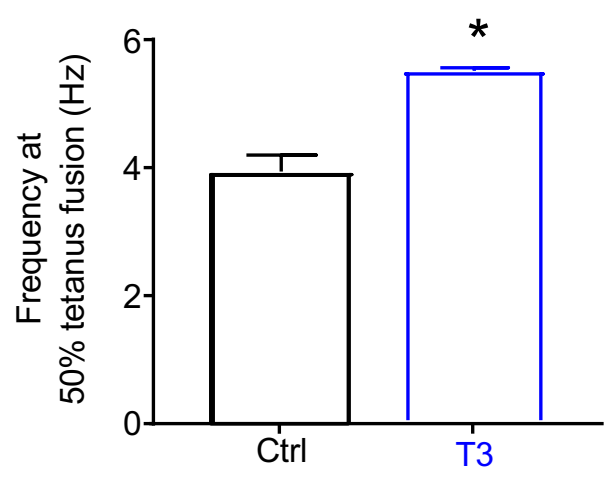

\section{Figure 27. Thyroid hormone elevates the tetanus threshold of human ESM.}

A, Fusion index calculated on representative traces of twitch tension generated by control (black) and +T3 (blue) ESM at $5 \mathrm{~Hz}$ tetanus stimulation. The fusion index calculated as the percentage ratio of the maximal relaxation amplitude before the last contraction of the tetanus $\left(\mathrm{C}_{\min }\right)$ to the amplitude of this last contraction $\left(\mathrm{C}_{\max }\right)$. $\mathbf{B}$, The fusion indexfrequency curve of control (black line) and $+\mathrm{T} 3$ (blue line) ESM. C, Stimulation frequency at $50 \%$ tetanus fusion of control (black bar) and $+\mathrm{T} 3$ (blue bar) ESM; $\mathrm{n}=8$ /group, ${ }^{*} \mathrm{p}<0.05$ by Student's t-test. 
We next asked if the T3 treatment affects the myosin heavy chain (MYH) isoform expression in ESM. Interestingly, T3 treatment clearly enhanced the abundance of adult fast MYH2 isoform with a reduction of the embryonic MYH3 isoform. The levels of the slow myosin isoform MYH7 were unchanged (Figure 28A, B). These molecular changes are well in line with the functional phenotype suggesting that T3 indeed supports maturation of fast skeletal muscle properties in ESM. The data also demonstrates that ESM respond to physiological stimuli comparable to skeletal muscle in vivo.

A

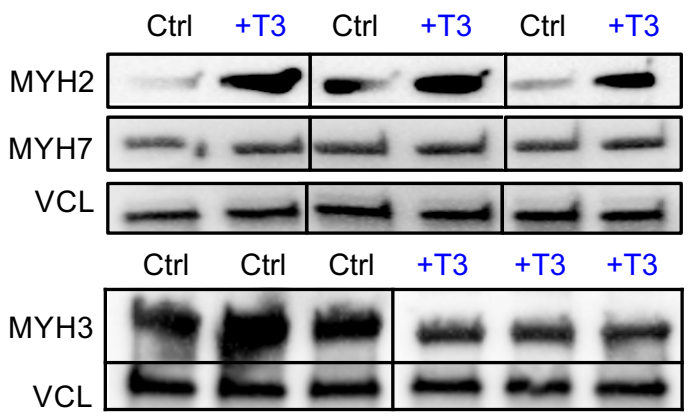

B

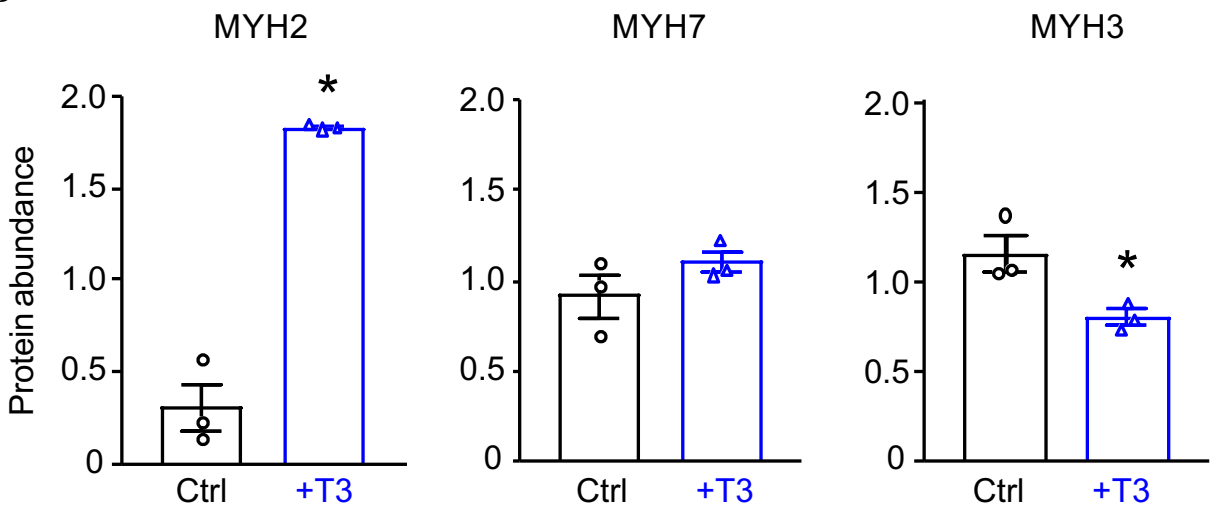

Figure 28. Myosin heavy chain isoform expression in human ESM treated with thyroid hormone.

A, Representative immunoblot for immunoreactive bands of fast myosin heavy chain (MYH2), slow myosin heavy chain (MYH7), embryonic myosin heavy chain (MYH3) and the loading control vinculin (VCL). B, Protein abundance of MYH2 (left panel), MYH7 (middle panel) and MYH3 (right panel) in 9 wks old ESM cultured with (blue bars) or without T3 (black bars); $\mathrm{n}=3$ /group, ${ }^{*} \mathrm{p}<0.05$ by Student's t-test. 


\subsection{Regenerative capacity of engineered skeletal muscle}

\subsubsection{Satellite cells in engineered skeletal muscle}

A fundamental property of skeletal muscle is the regenerative capacity by muscle stem cells called satellite cells (Bischoff 1975; Sambasivan et al. 2011). In a recent study from our lab, it was demonstrated that engineered skeletal muscle generated from rat myoblasts contains satellite cell niches that preserve regenerative capacity of ESM in vitro (Tiburcy et al. 2019).

To investigate whether human ESM would likewise have the capacity to regenerate in vitro, it was first scrutinized if ESM harbor stem cells resembling the native skeletal muscle's quiescent satellite cell population expressing transcription factor PAX7. Immunostaining identified a pool of cells with PAX7+ nuclei in ESM (13 $\pm 1 \%$ of total nuclei). $63 \pm 4 \%$ of the PAX7+ cells were adjacent to differentiated skeletal myofibers and positioned beneath a LAMININ+ basal lamina which is a characteristic of the in vivo satellite cell niche (Figure 29A). Of note, $75 \pm 6 \%$ of these PAX7+ cells were Ki67 negative, implicating a quiescent state (Figure 29B). 

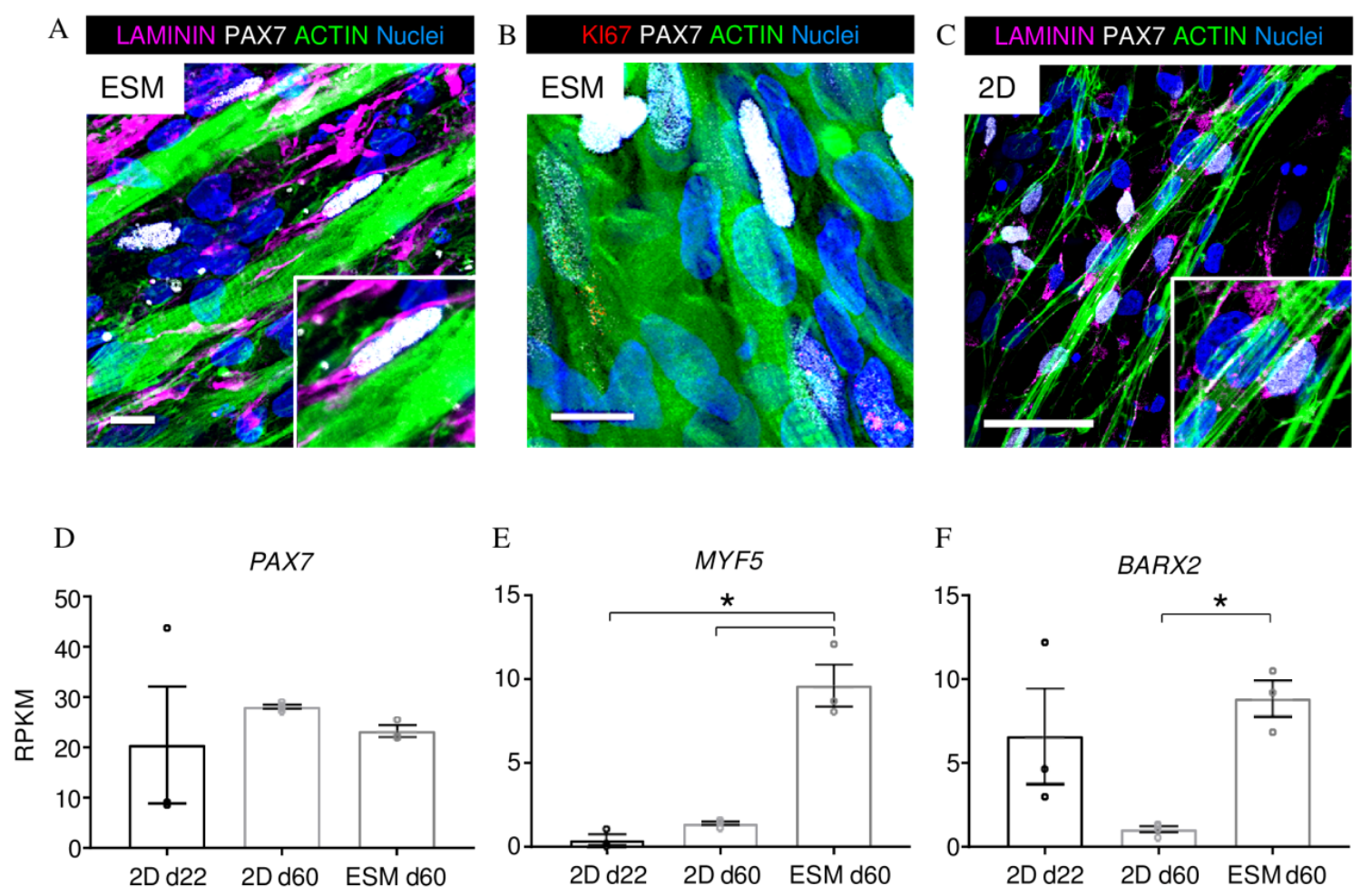

Figure 29. PAX7-positive cells adopt a satellite cell niche in human ESM.

Immunostaining of longitudinal sections of ESM for A, LAMININ (magenta), and B, KI67 (red), ACTIN (green), PAX7 (gray), and Nuclei (blue). Scale bars: $10 \mu \mathrm{m}$. C, Immunostaining of LAMININ (magenta), PAX7 (gray), ACTIN (green), and Nuclei (blue) in 2D monolayer cultures at day 60. Scale bar: $50 \mu \mathrm{m}$. D-F, RNA transcript (Reads per Kilobase Million, RPKM) of indicated muscle stem cell markers in 2D monolayer cells at day 22 and day 60, plus day 60 ESM; $n=3$ /group, * $<0.05$ by 1-way ANOVA and Tukey's multiple comparison test.

Same age 2D skeletal myocyte cultures prepared in parallel from the same stock of day 22 cells were compared for the presence of PAX7+ cells. While PAX7+ cells were identified on protein and transcript level in a similar quantity (Figure 29C,D), only $32 \pm 5 \%$ were associated with muscle fibers in $2 \mathrm{D}$ suggesting a higher propensity to resume a satellite cell position in $3 \mathrm{D}$. In line with this, higher expression of "mature" satellite cell markers MYF5 and BARX2 in ESM was found (Figure 29E,F) (Cornelison and Wold 1997; Meech et al. 2012). These data suggest that ESM may provide a favorable niche for the maintenance of the bona fide skeletal muscle stem cells in vitro. 


\subsubsection{Regenerative capacity of cardiotoxin injured ESM}

Finally, it was tested if the identified satellite-like cells in ESM can repair muscle in vitro. To induce muscle damage cardiotoxin (CTX $25 \mu \mathrm{g} / \mathrm{ml}$ ) was applied for 24 hours in 5 weeks old ESM (Figure 30A) according to Tiburcy et al., 2019.

48 hrs after CTX injury (day+2) ESM twitch tension could not be detected indicative of complete muscle destruction. The functional regenerative response was then assayed by tetanic force measurements. A partial but robust recovery of tetanic force (to $37 \pm 2 \%$ of initial force) was observed in ESM after 21 days of recovery (Figure 30B). In agreement with the recovery of function newly formed muscle fibers with organized sarcomeres were founded (Figure 30C).

To confirm that the recovery of contractile force was related to the function of proliferative, activated muscle stem cells, ESMs were irradiated with 30 Gray to prevent cell cycle activity before CTX injury (Tiburcy et al. 2019). It has been shown that irradiation does not affect the viability and function of matured postmitotic muscle fibers, but effectively inhibits cell cycle activity in muscle stem cells (Rosenblatt 1992; Tiburcy et al. 2019). Consistent with this observation the control tissue without CTX injury did not show a significant change in twitch tension after irradiation. In contrast, the injured tissue did not show any recovery of force, suggesting the regeneration by proliferating cells is effectively stopped (Figure 30B). In line with this, hardly any morphologically intact muscle cells were identified inside the ESM by immunostaining (Figure 30D). These data suggest a regenerative role of proliferative muscle stem cells inside ESM. 
A

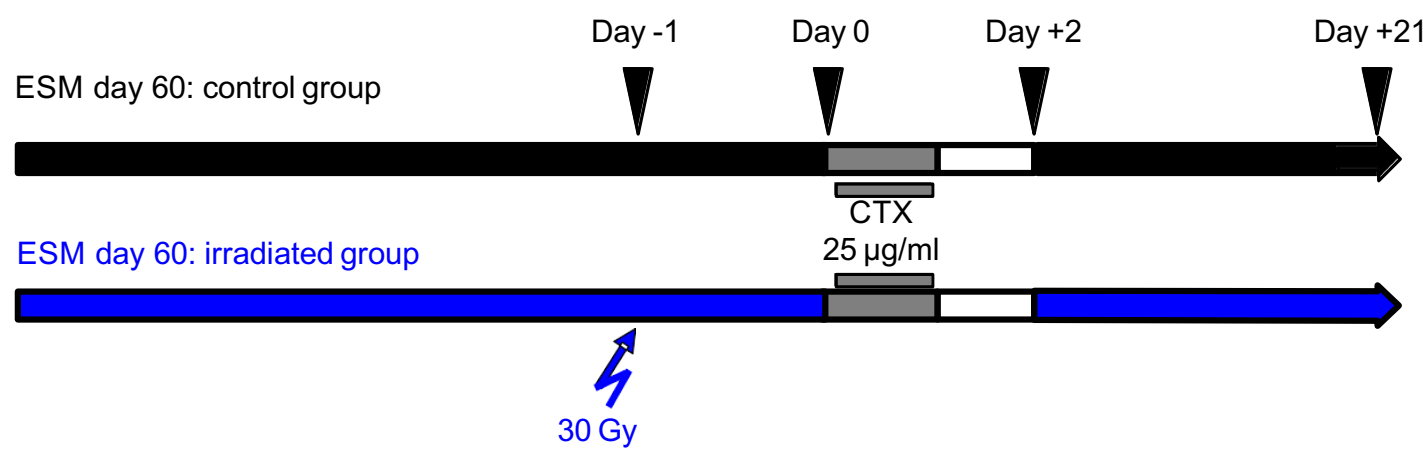

B

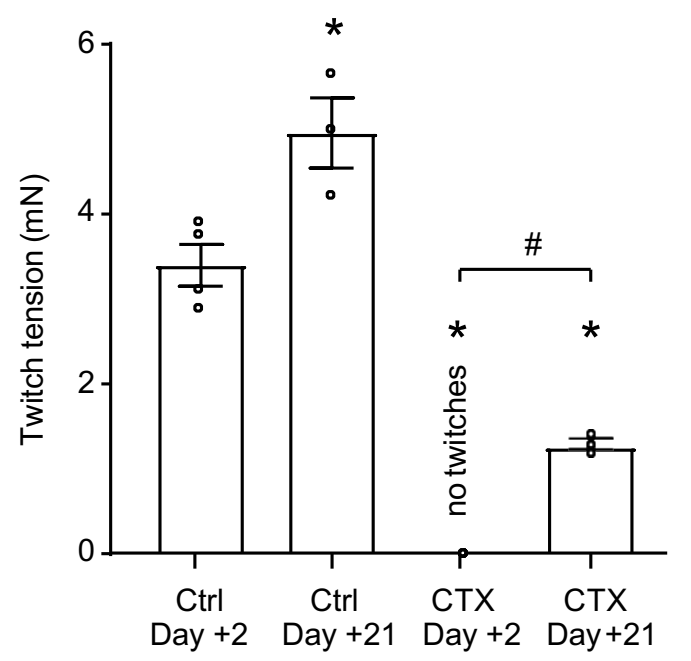

Control

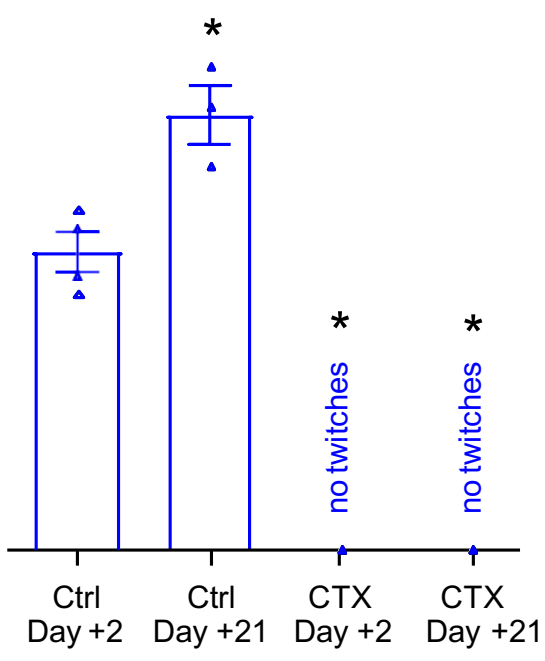

Irradiated

$\mathrm{C}$
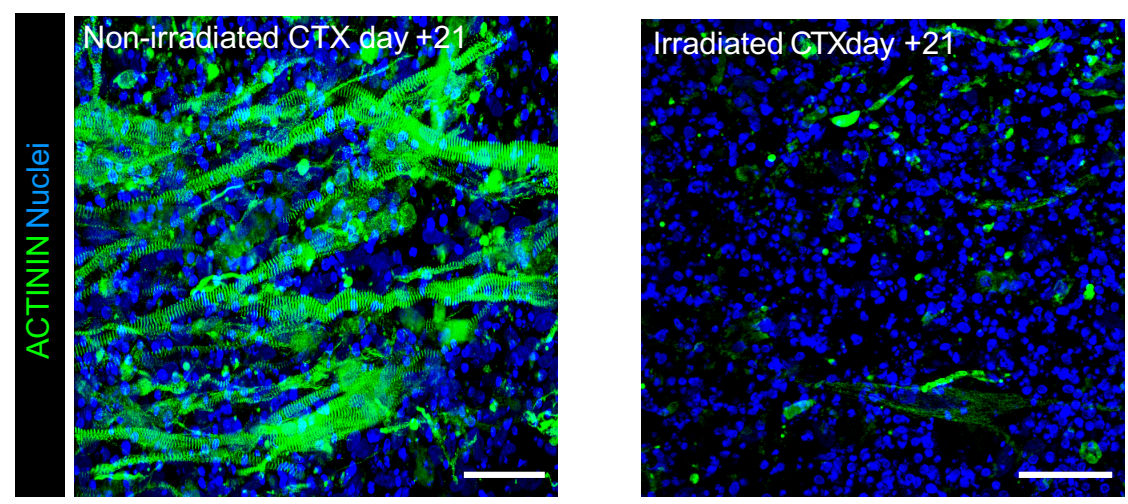

Figure 30. Functional regeneration of human engineered skeletal muscle.

A, Experimental design of cardiotoxin (CTX) injury model. ESM were incubated with $25 \mu \mathrm{g} / \mathrm{ml} \mathrm{CTX} \mathrm{for} 24 \mathrm{hrs}$. The irradiated group was treated with $30 \mathrm{~Gy} 24 \mathrm{hrs}$ before CTX injury (Tiburcy et al. 2019). B, Tetanic twitch tension at $100 \mathrm{~Hz}$ stimulation frequency of ESM without (black bars) or with irradiation (blue bars) at indicated time points after CTX $(25 \mu \mathrm{g} / \mathrm{ml})$ injury or control (Ctrl) condition; $\mathrm{n}=3-4 /$ group, ${ }^{*} \mathrm{p}<0.05$ vs. the respective Ctrl day +2 , by 1-way ANOVA and Tukey's multiple comparison test, \#*p<0.05 CTX day +2 vs. CTX day +21 . C, Immunostaining of sarcomeric $\alpha$-ACTININ (green) and Nuclei (blue) in non-irradiated ESM (left panel) and irradiated ESM (right panel) 21 days after CTX injury. Scale bars: $50 \mu \mathrm{m}$. 


\subsection{Modeling Duchenne Muscular Dystrophy in engineered skeletal muscle}

To evaluate ESM as a biologically relevant 3D platform to model skeletal myopathies, we hypothesized that dystrophin mutations associated with Duchenne Muscular Dystrophy (DMD) induce a pathological ESM phenotype.

To investigate this iPSC generated from a healthy individual (WT iPSC2) and two patients with frameshift mutations in the dystrophin $(D M D)$ gene were included in the study. The first DMD patient harbors an exon deletion (del; lacking exons 48 to 50) while the second DMD patient harbors a pseudoexon (pEx; pseudo-exon 47A leading to premature stop codon) (Long et al. 2018). Corresponding "myoedited"-DMD lines corrected by CRISPR/Cas9 mediated exon skipping were kindly provided by Eric N. Olson to serve as isogenic controls (Long et al. 2018). All lines were subjected to skeletal muscle differentiation (Figure 31A). Immunostaining on day 22 of differentiation illustrates a phenotypically comparable culture of elongated sarcomeric $\alpha$-ACTININ-positive skeletal myocytes in both DMD and myoedited-DMD cultures (Figure 31B). 
A

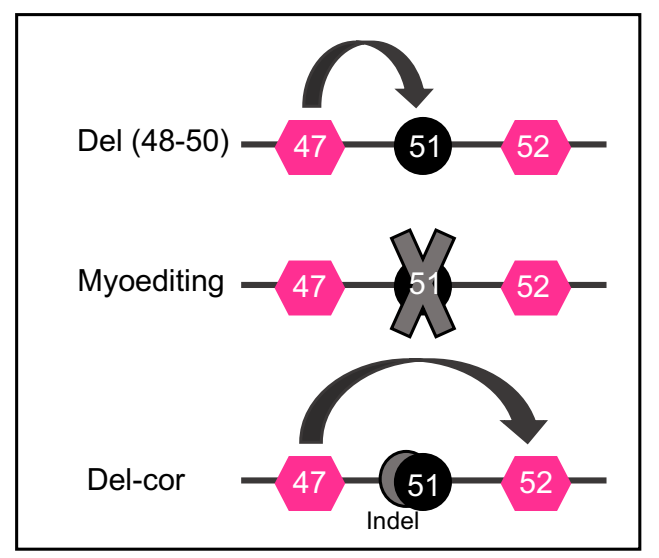

$\mathrm{C}$

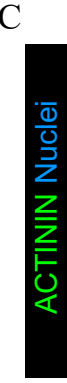

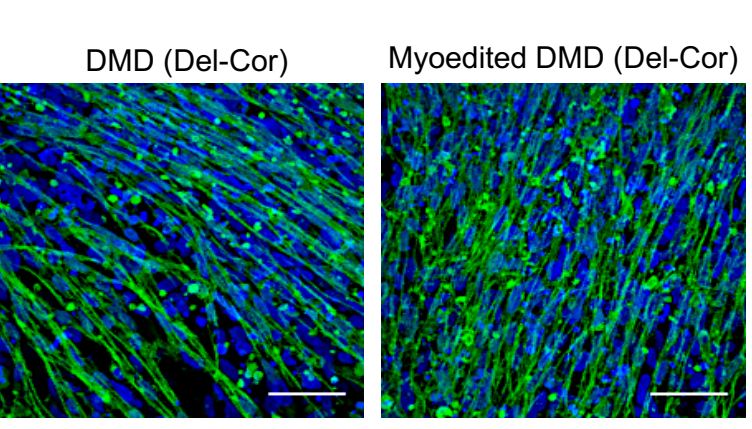

B

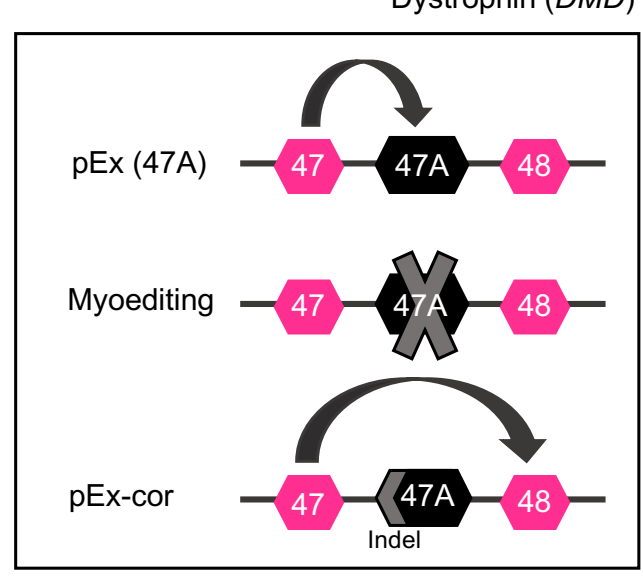

$\mathrm{D}$

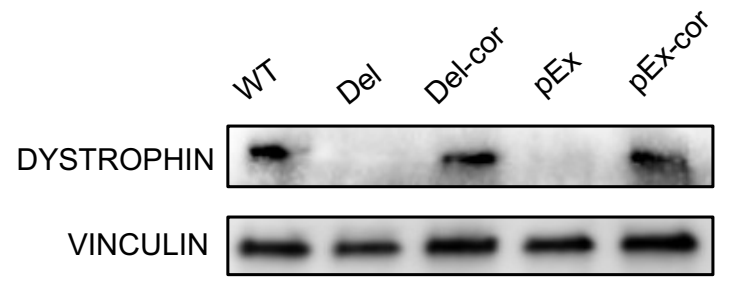

Figure 31. Efficient generation of skeletal myocytes from DMD and myoedited DMD iPSC lines.

A, Myoediting strategy in Del DMD iPSCs. In dystrophin (DMD) gene, deletion of exons 48 to 50 creates a frameshift mutation in exon 51 leading to premature stop codon in exon 51. By making use of the PAM sequences at the splice acceptor site of exon 51, the splice acceptor site was targeted by CRISPR/Cas9. Destruction of the splice acceptor site enabled efficient skipping of exon 51 thereby restoring the reading frame (adapted from Long et al. 2018). B, Myoediting strategy in pEx DMD iPSCs. In dystrophin $(D M D)$ gene, an intronic mutation between exon 47 and 48 creates an additional splice site leading to a pseudo exon 47A with a premature stop codon. Two guide RNAs were used to target pseudo-exon 47A $\mathrm{pEx}$ by CRISPR/Cas9. After destruction of the additional splice site the reading frame

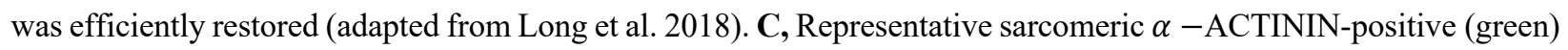
skeletal myocytes in 22 days old differentiated culture from DMD (Del) and myoedited DMD (Del-cor) iPSCs. Scale bar: $50 \mu \mathrm{m}$. D, Western blot analysis of WT, DMD (Del), myoedited DMD (Del-cor), DMD (pEx) and myoedited DMD (pEx-Cor) derived skeletal myocytes indicating the immunoreactive bands of dystrophin and Vinculin loading controls.

Western blot analysis on day 22 skeletal myocytes confirmed the absence of dystrophin protein in DMD cell lines, and restoration of dystrophin expression in myoedited-DMD skeletal myocytes

(Figure 31C). Accordingly, no dystrophin expression was observed in DMD-ESM (Figure 32). Re-expression of dystrophin with proper localization at the sub-sarcolemmal space was confirmed in ESM with genetic repair (Figure 32C,E). Importantly, contractile function was impaired in DMD-ESM $(0.7 \pm 0.2 \mathrm{mN}$ for DMD-Del ESM and 1.1 $\pm 0.4 \mathrm{mN}$ for DMD-pEx ESM at $100 \mathrm{~Hz}$ stimulation; Figure 33) and restored in myoedited ESM (4.7 $\pm 0.3 \mathrm{mN}$ for DMD-Del ESM and 2.6 $\pm 0.2 \mathrm{mN}$ for DMD-pEx at $100 \mathrm{~Hz}$ stimulation; Figure 33). 
A
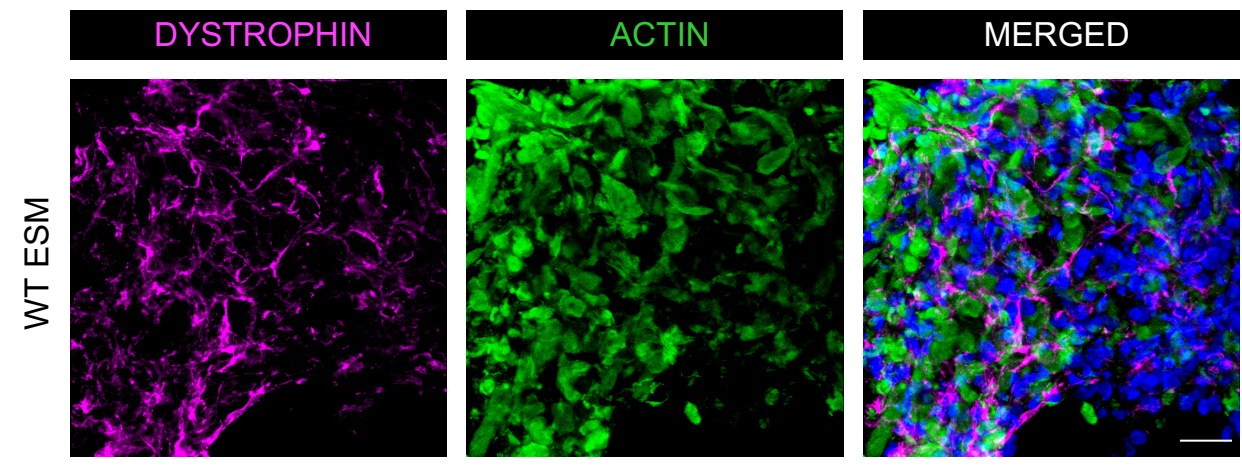

$\mathrm{B}$
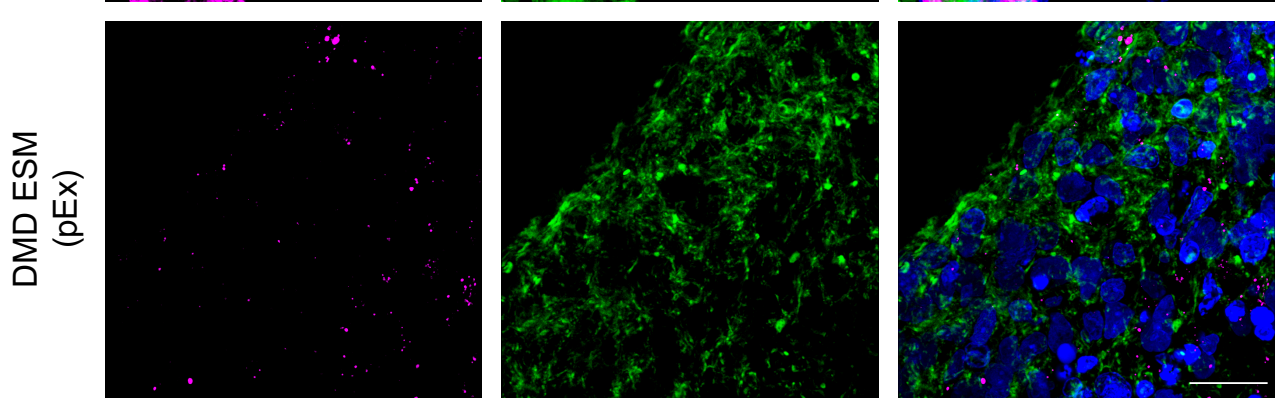

$\mathrm{C}$
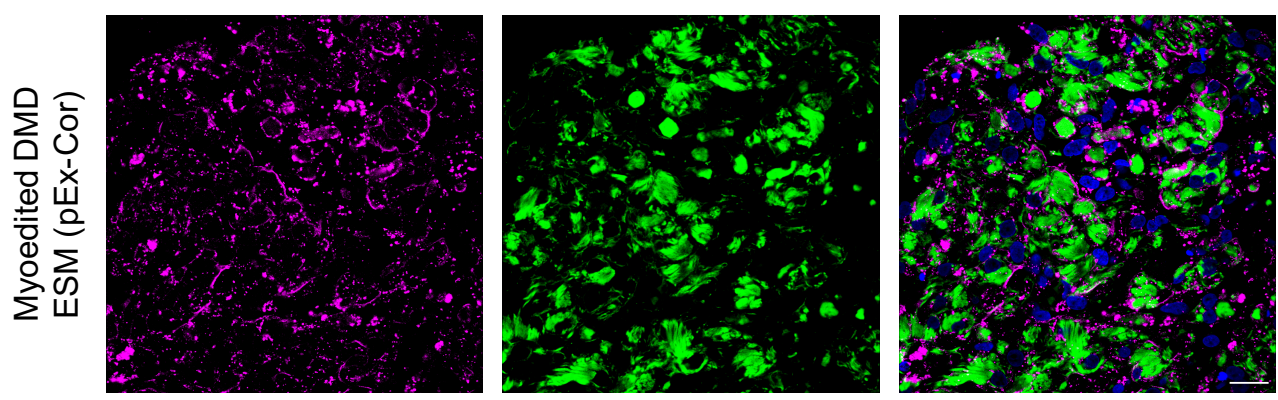

$\mathrm{D}$
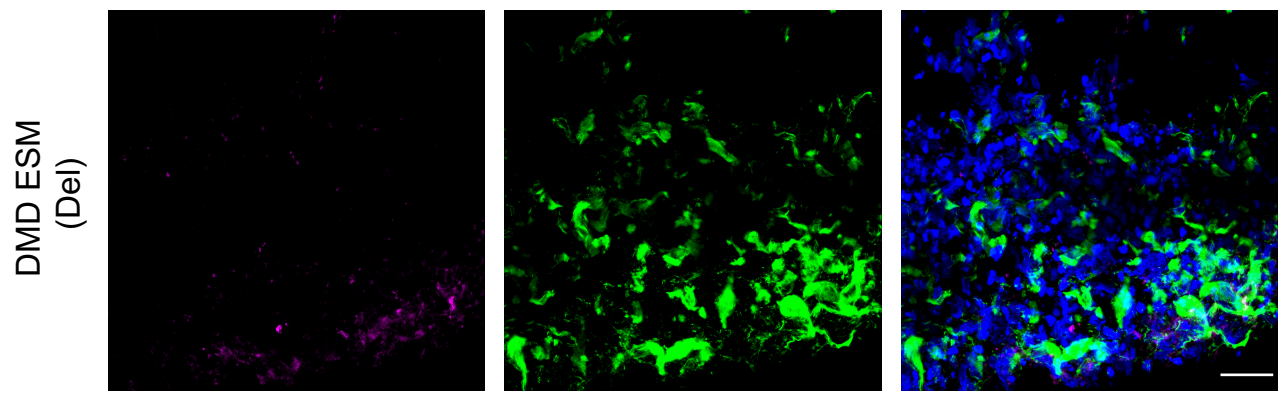

$\mathrm{E}$
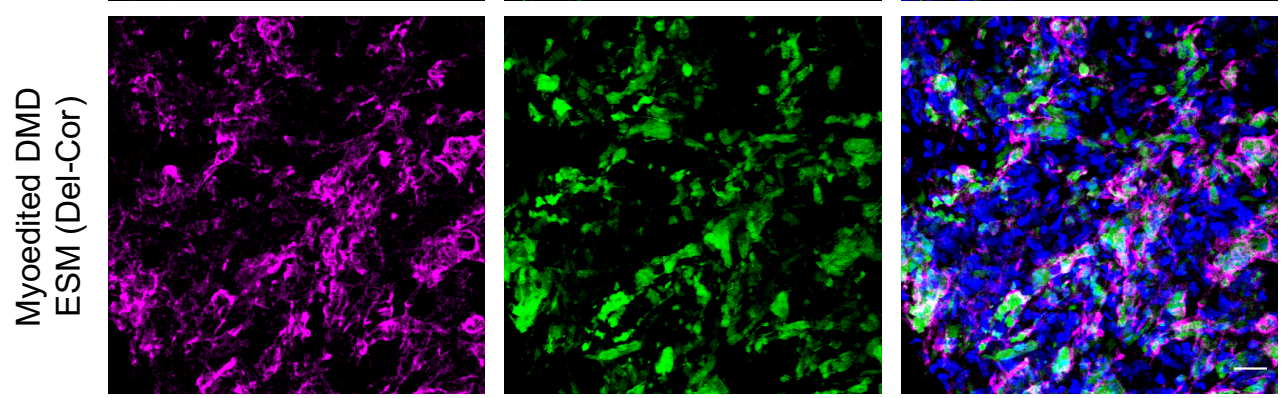

Figure 32. Restoration of Dystrophin expression in DMD ESM by myoediting.

Immunostaining of A, WT ESM, B, DMD ESM (pEx), C, myoedited DMD ESM (pEx-Cor), D, DMD ESM (Del) and E, myoedited DMD ESM (Del-Cor) cross sections for DYSTROPHIN (magenta), ACTIN (green) and Nuclei (blue); Scale bar: $20 \mu \mathrm{m}$. 


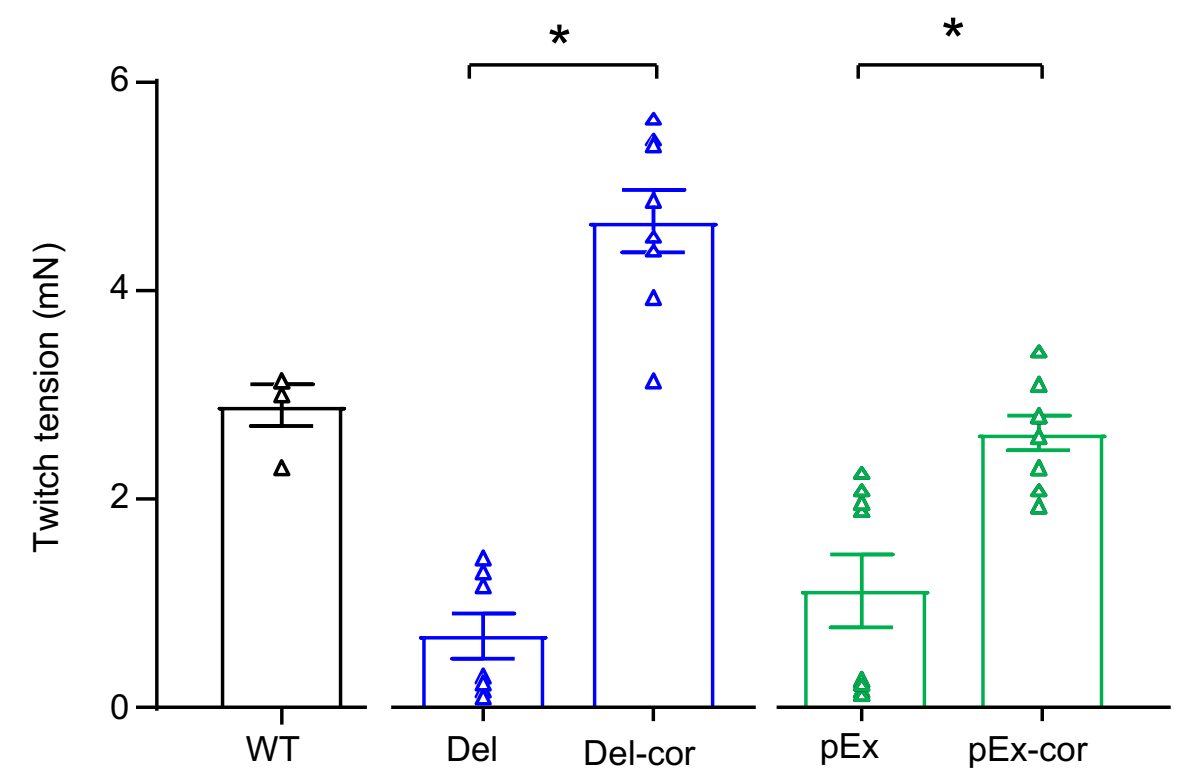

Figure 33. Rescue of DMD ESM dysfunction by myoediting.

Tetanic twitch tension at $100 \mathrm{~Hz}$ stimulation frequency generated by WT, DMD and myoedited DMD ESM. $\mathrm{n}=4$ WT ESM; $\mathrm{n}=7-8 / \mathrm{DMD}$ line, ${ }^{*} \mathrm{p}<0.05$ by Student's t-test. 


\section{Discussion}

Human development and disease are challenging to study because of limited access to patients and patient-derived material. While the use of animal models is valuable for studying physiology and pathophysiology in an in vivo context and for aiding pre-clinical development of therapeutics, animal models are costly, difficult to interrogate, and not always equivalent to human biology (Xi et al. 2017).

Innovative in vitro human cell-based models have the potential to overcome the problems with animal models and facilitate translation of findings from basic cellular research of human development and disease mechanisms into a patient context. It should be taken into consideration that cell-based models need to recapitulate the architecture, multicellular complexity and physiology of an organ but at the same time accommodate systematic experimental interventions. For these reasons, tissue engineering has emerged as an attractive technique to provide $3 \mathrm{D}$ cellbased models that recapitulate key aspects of in vivo tissue and organ complexity while being amenable to experimentation (Schmeichel and Bissell 2003).

Use of human PSCs for tissue engineering is expanding, and promising results have been obtained with human PSC-derived 3D tissues models of various organs including skeletal muscle. Still, the current human PSC-derived 3D models for skeletal muscle are criticized for immature properties of muscle fibers and modest physiological responsiveness (Maffioletti et al. 2018; Rao et al. 2018). Therefore, generating advanced human PSC-derived 3D models of skeletal muscle is instrumental for future translational applications.

By building on existing expertise of human PSC culture and tissue engineering (Soong et al. 2012; Tiburcy et al. 2017, 2019; Zimmermann et al. 2000) we aimed to generate engineered skeletal muscle (ESM) from human PSCs as a system for studying regeneration and modelling of muscular disease like Duchenne Muscular Dystrophy (DMD). Following this aim, the main achievements of this thesis are:

1) A robust, directed protocol for skeletal muscle differentiation of human wild-type and patient-specific iPSCs.

2) A close recapitulation of central stages of human skeletal muscle development in vitro. 
3) Feasibility of bioengineered skeletal muscle (BSM) organoid generation with characteristic skeletal muscle function.

4) ESM with physiological function from human PSC-derived skeletal myocytes under mechanical stimulation in serum free conditions. Typical skeletal muscle properties of ESM were demonstrated by:

i. a physiological myosin isoform switch in ESM treated with Triiodo-L-thyronine (T3)

ii. establishment of a satellite cell niche

iii. a robust regenerative response after cardiotoxin-induced injury

5) ESM as a disease model of Duchenne Muscular Dystrophy.

\subsection{Robust protocol for skeletal muscle differentiation of human iPSCs}

Despite the tremendous progress in developing protocols for directed differentiation of PSCs into skeletal myocytes (Chal et al. 2016; Choi et al. 2016), the challenges in directed differentiation of human PSCs toward a myogenic fate have hampered the development of robust in vitro models of skeletal muscle. This is likely due to the highly complex developmental process of skeletal myogenesis. To short cut these developmental processes most recent work has resorted to overexpression of myogenic regulatory factors like PAX7 in human PSC derived mesenchymal cells, a method pioneered in 1987 with the identification and overexpression of MYOD1 in nonmuscle cells (Davis, Weintraub, and Lassar 1987). Timed overexpression of PAX7 may allow for specific programming of non-muscle into muscle cells, however it is not clear how this may affect the function of resulting myocytes and tissues (Rao et al. 2018). Therefore, to provide a reproducible protocol for skeletal muscle differentiation from PSCs without genetic modifications, a precise appreciation of the specific signaling pathways involved is required.

The protocol reported here specifically directs human PSCs towards myogenic progenitors of limb muscle followed by primary and secondary skeletal myogenesis. Starting to establish the protocol in our lab, first two published protocols for directed skeletal muscle differentiation of human PSCs by Chal et al. 2016 and Choi et al. 2016 were tested without the desired outcome. Thus, we revisited the process of skeletal muscle development aiming at directing human PSCs to paraxial mesoderm progenitors under serum free culture conditions. To achieve this goal, we exposed human PSCs to CHIR99021 for Wnt activation, FGF2, and LDN-193189 for inhibition of BMP 
signaling. Wnt and FGF activation were applied to induce paraxial mesoderm formation (Boulet and Capecchi 2012; Chal et al. 2015; Ciruna and Rossant 2001; Yamaguchi et al. 1994). BMP inhibition was crucial to prevent the paraxial mesoderm progenitors from drifting to lateral mesoderm differentiation (Chal et al. 2016; Miura et al. 2006). An optimal paraxial mesoderm induction was the most critical step for a subsequent efficient skeletal myogenesis. Optimization efforts concentrated on finding an optimal initial cell density (resulting in an appropriate cell confluency of $30-40 \%$ ), as well as small molecule concentrations and exposure times for different PSC lines to obtain a homogenous culture of anterior paraxial mesoderm progenitors expressing specific markers including FOXC2 and MESP2. In the concurrent step, the differentiating culture was specifically directed towards generation of dermomyotomal progenitors of limb by removing the LDN-193189 from the culture to stop BMP inhibition and starting to use DAPT to inhibit Notch signaling. Notch inhibition is not only essential for dermomyotomal progenitor formation, but is also crucial to prevent the differentiation of anterior paraxial mesoderm progenitors to endothelial cells. The influence of Notch inhibition was obvious in our differentiation culture by antidromic expression of $P A X 3$ and FOXC2 (Choi et al. 2016; Mayeuf-Louchart et al. 2014). Following the myogenic program of limb skeletal muscle characterized by expression of specific markers including Sixl, in the next step HGF was used to promote the proliferation and differentiation of PAX3+ cells to a skeletal myogenic cell population (F. Bladt et al. 1995; Chal et al. 2016) including proliferative myogenic progenitors, myoblasts, spontaneously twitching myotubes and non-myocytes. The following figure summarizes the different tested protocols (Chal et al. 2016; Choi et al., 2016;). 
A Chal et al., 2016

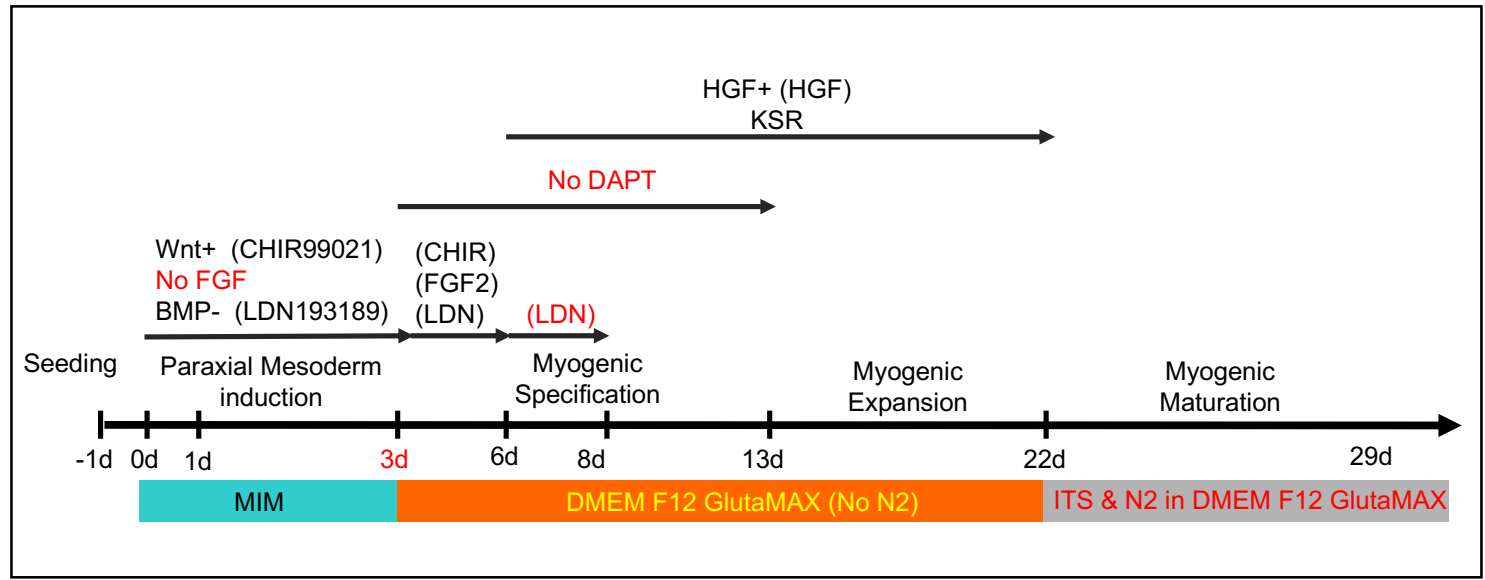

B Choi et al., 2016

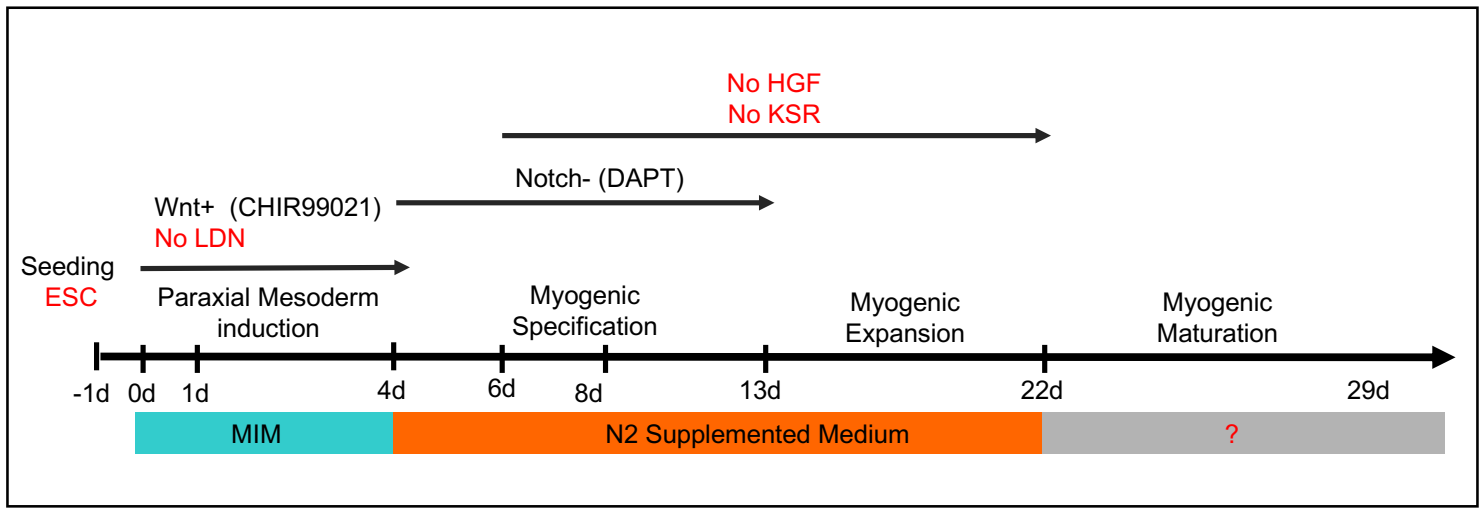

C This thesis

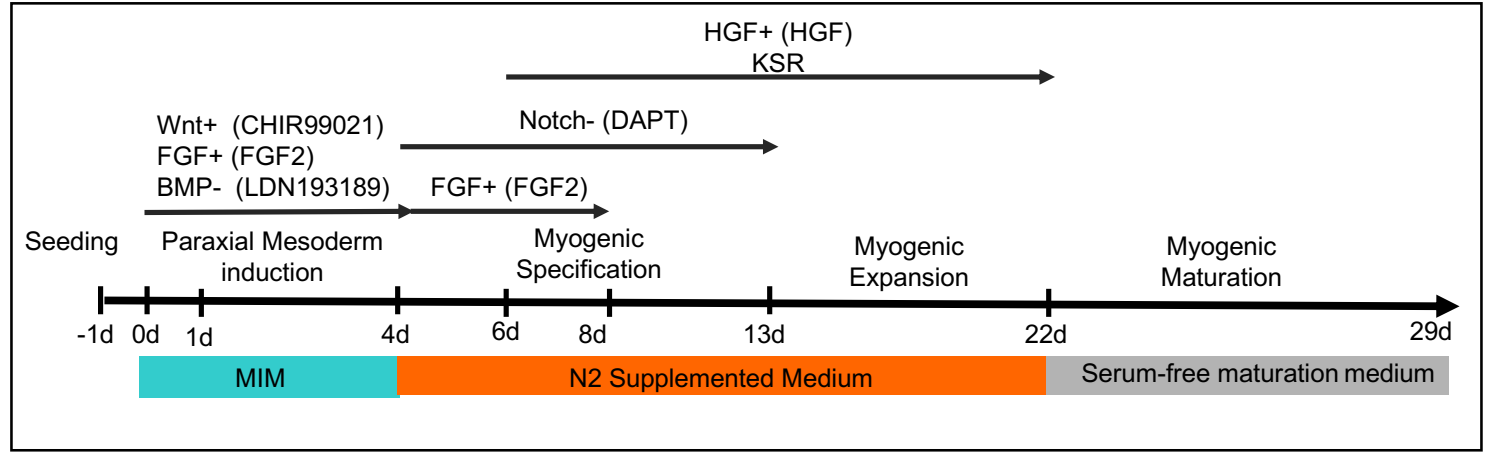

Figure 34. Summary of the tested skeletal myogenesis protocols.

Summary of the protocols for skeletal muscle differentiation from PSC optimized in A, Chal et al, 2016, B, Choi et al 2016 and $\mathbf{C}$, this thesis indicating the sequence and the timing of factor addition to modulate specific signaling pathways involved in skeletal myogenesis. Significant differences between protocols indicated in red. $+/-$ indicates pathway activation/inhibition, respectively. 
The developmental fidelity of our final protocol for skeletal muscle differentiation stems from the precise modulation of BMP, Notch and HGF signaling pathways to recapitulate the myogenic program of limb skeletal muscle and is underscored by the transcriptome data which showed great overlap to muscle development of the human embryo. Although Choi et al. 2016 took the advantage of using DAPT to inhibit Notch signaling, their protocol did not exploit (1) BMP inhibition for controlled paraxial mesoderm development and (2) HGF signaling activation for efficient promotion of skeletal myogenesis. In the protocol published by Chal et al. 2016 extended BMP inhibition in the absence of Notch inhibition directs the cells towards the trunk myogenic program rather than limb muscle (Pourquié et al. 2018). Importantly, the efficiency of our skeletal muscle differentiation protocol was high with significant numbers of Pax7, MyoD, and Myogeninpositive cells. Per input iPSC cell we obtained on average 34 skeletal muscle cells with 15 myogenic progenitors/myocytes, 14 mesenchymal progenitors, and 5 neurons. Flow cytometry confirmed the quantification of immunostaining data on day 22 of differentiation and showed comparable efficiency for 5 different iPSC lines indicating the robustness of the protocol.

\subsection{Engineered models of skeletal muscle from human PSCs}

In conventional 2D culture of human PSC-derived skeletal muscle fibers remain in an immature, embryonic phenotype which is vastly different compared to bona fide skeletal muscle. Culture systems to generate myogenic cells that are sufficiently mature to mimic the physiology of their counterparts in the intact organism are needed.

Only recently three studies reported the generation of skeletal muscle tissue from human pluripotent stem cells (Maffioletti et al. 2018; Rao et al. 2018; Xu et al. 2019) collectively suggesting a potential of 3D skeletal muscle for disease modeling and regenerative medicine. The contractile function output of these reported models is low compared to postnatal muscle and for the only transgene-free model published so far, muscle function was not reported (Maffioletti et al. 2018). No single strategy has yet been able to promote complete maturation of engineered constructs to a state indistinguishable from native adult human muscle tissue. 
In this thesis, the capacity of two different approaches to generate 3D models of skeletal muscle from human PSCs was tested:

\subsubsection{Bioengineered skeletal muscle (BSM) organoid from human PSCs}

For generation of BSM organoids undifferentiated iPSCs were embedded in a hydrogel of collagen type 1 and Matrigel ${ }^{\mathrm{TM}}$ and then subjected to the in this thesis established in vitro myogenesis protocol for skeletal muscle differentiation. Similar to the differentiation in 2D and in line with other published skeletal muscle, BSM organoids not only contained myocytes, but also non-muscle cells including neurons. A similar cell composition was recently reported in an alternative skeletal muscle organoid model (Faustino Martins et al. 2020). About $75 \%$ of the cross sectional area of BSM was populated by non-muscle cells in extracellular matrix. The clearly distinguishable muscle fibers showed a high degree of organized myofibrils. Importantly, BSM organoids developed organotypic contractile function with single twitches and tetanic contractions at high frequency stimulation. Further optimization is needed to increase the amount of muscle and to characterize the function of the non-muscle cells. Irrespective of the need for further optimization, BSM organoids recapitulate muscle development suggesting that BSM can be applied to model human developmental biology.

\subsubsection{Engineered skeletal muscle from human PSC-derived skeletal muscle cells}

Based on previous work in the rat (Tiburcy et al. 2019), we demonstrate that Engineered Skeletal Muscle (ESM) from human iPSC-derived skeletal myocytes supports physiological skeletal muscle function and provides a regenerative satellite cell niche in vitro. In contrast to BSM organoids, ESMs are generated from enzymatically dissociated day 22 monolayer PSC-derived skeletal muscle cells. This allows precise control over the cellular input and composition of the ESM. After optimizing ESM conditions, the skeletal muscle cell content of ESM was 2.5 fold higher than in BSM organoids and functional output (maximal tetanic twitch tension) was 2-2.5 fold higher in ESM compared to BSM organoids of identical time in culture (28 days maturation). 
The use of 22 days old skeletal myocytes for further physiological development of muscle fibers in ESM was based on the immunostaining and transcriptome data showing that day 22 cells contained a high number of PAX7+ myogenic progenitors among narrow aligned primary myotubes indicative of the primary myogenesis stage. We reasoned that this stage would be optimal to use in ESM to stimulate further fusion in 3D and to aid secondary myogenesis (Biressi et al. 2007; Buckingham and Relaix 2007). The continuum of muscle development with fetal stage secondary myogenesis in ESM was evident by expression of NFIX, CKM, and MYH8 (Supplemental Figure 1).

Further evidence for continuing physiological development in ESM were obtained by immunostaining showing a highly organized muscle fiber syncytium surrounded by sarcolemma with properly localized Dystrophin-associated protein complexes. A mature ultrastructure containing sarcomeres with M-bands, t-tubules, and organized mitochondria further supports an advanced maturation. Finally, contractile parameters are in agreement with fetal muscle development (Racca et al. 2013). It should be noted however, that ESM is still in a maturing state of development as evidenced by the small average muscle cell diameter, the myosin isoform pattern, the absolute force development (3-10\% of adult muscle), and higher numbers of progenitor cells. Strategies to enhance physiological hypertrophic growth are still needed to further improve ESM.

\subsection{Myosin isoform switch in ESM under Triiodo-L-thyronine exposure}

There are different approaches to define the maturation state of skeletal muscle fibers. Final fiber type of a given muscle is mainly determined by developmental history, physiological properties and innervation. Physiologically the skeletal muscle fibers are classified according to twitch speed and primary ATP production patterns (Schiaffino and Reggiani 2011). Myosin heavy chain (MYH), the most abundant motor protein in human skeletal muscle, is an important intrinsic determinant of muscle twitch (Baldwin and Haddad 2001). The expression of MYH isoforms follows a developmental sequence, with the embryonic (MYH3) and slow MYH (MYH7) being the first to be expressed. Fetal and neonatal fibers transiently express perinatal MYH (MYH8), while the fast isoforms start to be expressed during late fetal myogenesis. Adult muscle fibers of 
limb and trunk skeletal muscle express specific isoforms of MYH. Adult slow twitch fiber type I expresses slow MYH7, whereas adult fast twitch fibers type IIa and IIx express fast MYH2 and fast MYH1 respectively (Schiaffino and Reggiani 2011).

A major factor involved in postnatal muscle fiber type differentiation is the level of thyroid hormone (triiodothyronine), which is low until birth, then increases to reach its maximum level at 2 to 3 weeks after birth. Triiodothyronine (T3), independent of the neuronal innervation, has a role in the postnatal transition from embryonic and neonatal to adult MYHs. Moreover, T3 stimulates slow-to-fast muscle fiber type conversion by inducing the transition of MYH7 to MYH2 and MYH2 to MYH1 (Schiaffino and Reggiani 2011; Simonides and van Hardeveld 2008).

The influence of thyroid hormone has not been investigated in a human in vitro model of skeletal muscle so far. Here, we show that the ESM model responds physiologically to T3 treatment. Functional analysis of the ESM revealed that T3 treatment increased the speed of contraction (T1) of single twitches at early and late maturation stages as well as the speed of relaxation (T2) at late maturation and subsequently shifted the tetanus threshold to higher frequencies. By T3 treatment the expression pattern of MYH in ESM showed characteristic patterns in line with the functional changes. While expression level of adult fast MYH2 was significantly increased, no significant change was found in the expression of adult slow MYH7. More importantly, T3 induced a significant decrease in expression level of embryonic MYH, collectively shifting the myosin isoform pattern to a more mature fast muscle pattern. These findings establish that the supplementation of T3 is fundamental for the routine culture of engineered muscle.

\subsection{Regenerative potential of ESM in response to cardiotoxin-induced injury}

Adult skeletal muscle has a remarkable capability for self-repair and regeneration after injury. Skeletal muscle stem cells (satellite cells) are the main source of regeneration in injured muscle. Satellite cells are mitotically quiescent, located under the basal lamina in an intimate association with plasma membrane of adult muscle fiber. Satellite cells are specifically characterized by expression of PAX7 and they have the potential for cell cycle activation, myogenic differentiation and functional regeneration upon injury (Sambasivan et al. 2011; Seale et al. 2000). 
Recently, our group confirmed that a satellite cell niche with true muscle stem cell properties and regenerative capacity can be preserved in rat ESM (Tiburcy et al. 2019) supporting earlier studies on the regeneration potential of tissue engineered muscle (Juhas et al. 2014). In this study we provide evidence that human PSC-derived skeletal muscle fibers generated in our ESM model are mature enough to provide a proper niche for PAX7+/Ki67- quiescent satellite cells. The majority but not all of PAX7+ cells were situated adjacent to a muscle fiber. The non-adjacent PAX7+ cells resemble the proliferating progenitor cells giving rise to developing fetal muscle fibers in ESM.

It has been demonstrated that cardiotoxin (CTX)-induced injury is a feasible method to preferentially damage the sarcomeric structure of muscle fibers in rat skeletal engineered skeletal muscle, resulting in full contractile failure without affecting the satellite cell niche (Tiburcy et al. 2019). Therefore, to evaluate the regenerative potential of the satellite cells in human ESM, the CTX injury method was adapted to our model. Approximately 37\% of contractile function of the human ESM was regained after 3 weeks of regeneration by injured human ESM. To verify this outcome, CTX injury was applied to irradiated human ESM (Heslop, Morgan, and Partridge 2000; Tiburcy et al. 2019; Wakeford, Watt, and Partridge 1991). The fact that the contractile function of irradiated, mitotically inactivated human ESM was not regenerated in response to CTX injury, confirmed the regenerative potential of the satellite cell niche (by its activation from a quiescent to a proliferative state) of human PSC-derived skeletal muscle fibers in ESM model. While these data provide a proof-of-principle for satellite cell-based regeneration it remains to be established whether in vitro activation recapitulates in vivo processes and whether a complete regeneration with $100 \%$ recovery of force can be achieved in vitro. One important aspect could be the presence or absence of supporting cells for regeneration. For example, macrophages have been shown to support muscle regeneration (Juhas et al. 2018; Shang et al. 2020). By addition of macrophages to the ESM formulation, this will be tested in future studies.

\subsection{Modeling Duchenne Muscular Dystrophy in ESM}

Since several muscular dystrophies, including Duchenne Muscular Dystrophy (DMD) develop in in postnatal life, in vitro models of human skeletal muscle tissue comprising of embryonic cells 
may be inappropriate to accurately recapitulate the phenotypes of DMD patients (Nam et al. 2015; Smith et al. 2016).

Here, we hypothesized that i) using our established 2D differentiation protocol, DMD iPSCs can be differentiated to skeletal myocytes and ii) ESM model has the capacity to generate sufficiently mature human DMD iPSC-derived skeletal muscle fibers mimicking the muscle pathophysiology of DMD. In comparison with WT ESM, ESM generated from human DMD iPSC-derived skeletal myocytes (DMD ESM) showed contractile weakness. This lack of function corresponded to the absence of dystrophin localized to the sarcolemma of skeletal muscle fibers in DMD ESM. Of note, the degree of skeletal muscle weakness was different in the two lines, with a less severe phenotype in the DMD pEx line. This is in line with contractile function data from Engineered Heart Muscle (EHM) and may also reflect the severity of disease in the patient (Long et al 2018). Interestingly, restoring the dystrophin expression through myoediting, a CRIPSR/Cas9 based method pioneered by Eric N. Olson and colleagues (Long et al. 2018), could effectively rescue the contractile function of ESM generated from myoedited skeletal myocytes (corrected DMD ESM). These data demonstrate that human ESM generated from DMD induced PSC-derived skeletal myocytes can serve as a preclinical platform for disease modeling. 


\section{Summary and perspectives}

In this project, we provide a robust transgene-free protocol for skeletal muscle differentiation of human pluripotent stem cells (PSCs) in a serum-free culture followed by generation of maturing skeletal muscle fibers in novel 3D models of skeletal muscle, namely ESM and BSM organoids. Molecular and morphological analyses demonstrate the stage specific recapitulation of skeletal muscle development. Creatine supplementation enhanced the contractile function of mechanically conditioned ESM. Thyroid hormone (T3) treatment elevated the maturation level of PSC-derived skeletal muscle fibers in ESM. Functionality of the apparent niche for skeletal muscle satellite cells was documented by regeneration of contractile function after cardiotoxin (CTX)-injury. Modelling Duchenne Muscular Dystrophy (DMD) in ESM provided "proof-of-concept" for disease modeling and therapeutic genome editing by CRISPR/Cas9 based exon skipping. According to these findings, we propose ESM as a high fidelity in vitro model of skeletal muscle addressing the need for robust human preclinical models of skeletal muscle disease. This methodology may in addition lay the groundwork for the development of novel therapies targeting skeletal muscle dysfunction, muscle regeneration and muscle loss. 


\section{Bibliography}

Afshar Bakooshli, Mohsen, Ethan S. Lippmann, Ben Mulcahy, Nisha Iyer, Christine T. Nguyen, Kayee Tung, Bryan A. Stewart, Hubrecht van den Dorpel, Tobias Fuehrmann, Molly Shoichet, Anne Bigot, Elena Pegoraro, Henry Ahn, Howard Ginsberg, Mei Zhen, Randolph Scott Ashton, and Penney M. Gilbert. 2019. "A 3D Culture Model of Innervated Human Skeletal Muscle Enables Studies of the Adult Neuromuscular Junction.” ELife 8:e44530. doi: $10.7554 /$ eLife.44530.

Allbrook, D. B., M. F. Han, and A. E. Hellmuth. 1971. "Population of Muscle Satellite Cells in Relation to Age and Mitotic Activity." Pathology 3(3):233-43. doi: 10.1080/00313027109073739.

Allen, Ronald E., Shannon M. Sheehan, Richard G. Taylor, Teresa L. Kendall, and Glenna M. Rice. 1995. "Hepatocyte Growth Factor Activates Quiescent Skeletal Muscle Satellite Cells in Vitro." Journal of Cellular Physiology 165(2):307-12. doi: 10.1002/jcp.1041650211.

Al-Qusairi, Lama, and Jocelyn Laporte. 2011. "T-Tubule Biogenesis and Triad Formation in Skeletal Muscle and Implication in Human Diseases." Skeletal Muscle 1(1):26. doi: 10.1186/2044-5040-1-26.

Amoasii, Leonela, John C. W. Hildyard, Hui Li, Efrain Sanchez-Ortiz, Alex Mireault, Daniel Caballero, Rachel Harron, Thaleia-Rengina Stathopoulou, Claire Massey, John M. Shelton, Rhonda Bassel-Duby, Richard J. Piercy, and Eric N. Olson. 2018. "Gene Editing Restores Dystrophin Expression in a Canine Model of Duchenne Muscular Dystrophy." Science (New York, N.Y.) 362(6410):86-91. doi: 10.1126/science.aau1549.

Amoasii, Leonela, Chengzu Long, Hui Li, Alex A. Mireault, John M. Shelton, Efrain SanchezOrtiz, John R. McAnally, Samadrita Bhattacharyya, Florian Schmidt, Dirk Grimm, Stephen D. Hauschka, Rhonda Bassel-Duby, and Eric N. Olson. 2017. "Single-Cut Genome Editing Restores Dystrophin Expression in a New Mouse Model of Muscular Dystrophy." Science Translational Medicine 9(418). doi: 10.1126/scitranslmed.aan8081.

Aulehla, Alexander, and Olivier Pourquié. 2008. "Oscillating Signaling Pathways during Embryonic Development." Current Opinion in Cell Biology 20(6):632-37. doi: 10.1016/j.ceb.2008.09.002.

Baghbaderani, Behnam Ahmadian, Adhikarla Syama, Renuka Sivapatham, Ying Pei, Odity Mukherjee, Thomas Fellner, Xianmin Zeng, and Mahendra S. Rao. 2016. "Detailed Characterization of Human Induced Pluripotent Stem Cells Manufactured for Therapeutic 
Applications." Stem Cell Reviews and Reports 12(4):394-420. doi: 10.1007/s12015-0169662-8.

Baldwin, K. M., and F. Haddad. 2001. "Effects of Different Activity and Inactivity Paradigms on Myosin Heavy Chain Gene Expression in Striated Muscle." Journal of Applied Physiology (Bethesda, Md.: 1985) 90(1):345-57. doi: 10.1152/jappl.2001.90.1.345.

Becht, Etienne, Leland McInnes, John Healy, Charles-Antoine Dutertre, Immanuel W. H. Kwok, Lai Guan Ng, Florent Ginhoux, and Evan W. Newell. 2018. "Dimensionality Reduction for Visualizing Single-Cell Data Using UMAP." Nature Biotechnology. doi: $10.1038 /$ nbt.4314.

Bentzinger, C. F., Y. X. Wang, and M. A. Rudnicki. 2012. "Building Muscle: Molecular Regulation of Myogenesis." Cold Spring Harbor Perspectives in Biology 4(2):a008342a008342. doi: 10.1101/cshperspect.a008342.

Biressi, Stefano, Mario Molinaro, and Giulio Cossu. 2007. "Cellular Heterogeneity during Vertebrate Skeletal Muscle Development.” Developmental Biology 308(2):281-93. doi: 10.1016/j.ydbio.2007.06.006.

Bischoff, R. 1975. "Regeneration of Single Skeletal Muscle Fibers in Vitro." The Anatomical Record 182(2):215-35. doi: 10.1002/ar.1091820207.

Bladt, F., D. Riethmacher, S. Isenmann, A. Aguzzi, and C. Birchmeier. 1995. "Essential Role for the C-Met Receptor in the Migration of Myogenic Precursor Cells into the Limb Bud." Nature 376(6543):768-71. doi: 10.1038/376768a0.

Bladt, Friedhelm, Dieter Riethmacher, Stefan Isenmann, Adriano Aguzzi, and Carmen Birchmeier. 1995. "Essential Role for the C- Met Receptor in the Migration of Myogenic Precursor Cells into the Limb Bud." Nature 376(6543):768-71. doi: 10.1038/376768a0.

Boergermann, J. H., J. Kopf, P. B. Yu, and P. Knaus. 2010. "Dorsomorphin and LDN-193189 Inhibit BMP-Mediated Smad, P38 and Akt Signalling in C2C12 Cells." The International Journal of Biochemistry \& Cell Biology 42(11):1802-7. doi: 10.1016/j.biocel.2010.07.018.

Bonilla, Diego A., and Yurany Moreno. 2015. "Molecular and Metabolic Insights of Creatine Supplementation on Resistance Training." Revista Colombiana de Quimica 44(1):11-18. doi: 10.15446/rev.colomb.quim.v44n1.53978.

Borycki, A. G., B. Brunk, S. Tajbakhsh, M. Buckingham, C. Chiang, and C. P. Emerson. 1999. "Sonic Hedgehog Controls Epaxial Muscle Determination through Myf5 Activation." Development (Cambridge, England) 126(18):4053-63. 
Boulet, Anne M., and Mario R. Capecchi. 2012. "Signaling by FGF4 and FGF8 Is Required for Axial Elongation of the Mouse Embryo." Developmental Biology 371(2):235-45. doi: 10.1016/j.ydbio.2012.08.017.

Buckingham, Margaret, Lola Bajard, Ted Chang, Philippe Daubas, Juliette Hadchouel, Sigolène Meilhac, Didier Montarras, Didier Rocancourt, and Frédéric Relaix. 2003. "The Formation of Skeletal Muscle: From Somite to Limb.” Journal of Anatomy 202(1):59-68. doi: 10.1046/j.1469-7580.2003.00139.x.

Buckingham, Margaret, and Frédéric Relaix. 2007. "The Role of Pax Genes in the Development of Tissues and Organs: Pax3 and Pax7 Regulate Muscle Progenitor Cell Functions." Annual Review of Cell and Developmental Biology 23(1):645-73. doi: 10.1146/annurev.cellbio.23.090506.123438.

Buller, A. J., and D. M. Lewis. 1965. "The Rate of Tension Development in Isometric Tetanic Contractions of Mammalian Fast and Slow Skeletal Muscle." The Journal of Physiology 176(3):337-54. doi: 10.1113/jphysiol.1965.sp007554.

Butler-Browne, G. S., J. P. Barbet, and L. E. Thornell. 1990. "Myosin Heavy and Light Chain Expression during Human Skeletal Muscle Development and Precocious Muscle Maturation Induced by Thyroid Hormone." Anatomy and Embryology 181(6):513-22. doi: 10.1007/BF00174624.

Celichowski, J. 2000. "Mechanisms Underlying the Regulation of Motor Unit Contraction in the Skeletal Muscle." Journal of Physiology and Pharmacology: An Official Journal of the Polish Physiological Society 51(1):17-33.

Chal, Jérome, Ziad Al Tanoury, Marie Hestin, Bénédicte Gobert, Suvi Aivio, Aurore Hick, Thomas Cherrier, Alexander P. Nesmith, Kevin K. Parker, and Olivier Pourquié. 2016. "Generation of Human Muscle Fibers and Satellite-like Cells from Human Pluripotent Stem Cells in Vitro." Nature Protocols 11(10):1833-50. doi: 10.1038/nprot.2016.110.

Chal, Jérome, Alexis Hubaud, Agata Bera, Olga Sumara, Jean-Marie Garnier, Leif Kennedy, Marie Knockaert, Barbara Gayraud-Morel, Shahragim Tajbakhsh, and Olivier Pourquié. 2018. "Recapitulating Early Development of Mouse Musculoskeletal Precursors of the Paraxial Mesoderm in Vitro." Development 145(6). doi: 10.1242/dev.157339.

Chal, Jérome, Masayuki Oginuma, Ziad Al Tanoury, Bénédicte Gobert, Olga Sumara, Aurore Hick, Fanny Bousson, Yasmine Zidouni, Caroline Mursch, Philippe Moncuquet, Olivier Tassy, Stéphane Vincent, Ayako Miyanari, Agata Bera, Jean-Marie Garnier, Getzabel Guevara, Marie Hestin, Leif Kennedy, Shinichiro Hayashi, Bernadette Drayton, Thomas Cherrier, Barbara Gayraud-Morel, Emanuela Gussoni, Frédéric Relaix, Shahragim 
Tajbakhsh, and Olivier Pourquié. 2015. "Differentiation of Pluripotent Stem Cells to Muscle Fiber to Model Duchenne Muscular Dystrophy." Nature Biotechnology 33(9):96269. doi: $10.1038 /$ nbt.3297.

Chal, Jérome, and Olivier Pourquié. 2009. "Patterning and Differentiation of the Vertebrate Spine.” Cold Spring Harbor Monograph Archive 53(0):41-116. doi: 10.1101/0.41-116.

Chal, Jérome, and Olivier Pourquié. 2017. "Making Muscle: Skeletal Myogenesis in Vivo and in Vitro." Development 144(12):2104-22. doi: 10.1242/dev.151035.

Choi, In Young, HoTae Lim, Kenneth Estrellas, Jyothi Mula, Tatiana V. Cohen, Yuanfan Zhang, Christopher J. Donnelly, Jean-Philippe Richard, Yong Jun Kim, Hyesoo Kim, Yasuhiro Kazuki, Mitsuo Oshimura, Hongmei Lisa Li, Akitsu Hotta, Jeffrey Rothstein, Nicholas Maragakis, Kathryn R. Wagner, and Gabsang Lee. 2016. "Concordant but Varied Phenotypes among Duchenne Muscular Dystrophy Patient-Specific Myoblasts Derived Using a Human IPSC-Based Model." Cell Reports 15(10):2301-12. doi: 10.1016/j.celrep.2016.05.016.

Cinnamon, Y., N. Kahane, I. Bachelet, and C. Kalcheim. 2001. "The Sub-Lip Domain--a Distinct Pathway for Myotome Precursors That Demonstrate Rostral-Caudal Migration." Development 128(3):341-51.

Ciruna, B., and J. Rossant. 2001. "FGF Signaling Regulates Mesoderm Cell Fate Specification and Morphogenetic Movement at the Primitive Streak." Developmental Cell 1(1):37-49. doi: 10.1016/s1534-5807(01)00017-x.

Conboy, Irina M., and Thomas A. Rando. 2002. "The Regulation of Notch Signaling Controls Satellite Cell Activation and Cell Fate Determination in Postnatal Myogenesis." Developmental Cell 3(3):397-409. doi: 10.1016/s1534-5807(02)00254-x.

Cooke, Matthew B., Emma Rybalka, Andrew D. Williams, Paul J. Cribb, and Alan Hayes. 2009. "Creatine Supplementation Enhances Muscle Force Recovery after Eccentrically-Induced Muscle Damage in Healthy Individuals." Journal of the International Society of Sports Nutrition 6:13. doi: 10.1186/1550-2783-6-13.

Cornelison, D. D., and B. J. Wold. 1997. "Single-Cell Analysis of Regulatory Gene Expression in Quiescent and Activated Mouse Skeletal Muscle Satellite Cells." Developmental Biology 191(2):270-83. doi: 10.1006/dbio.1997.8721.

Cutts, A. 1988. "The Range of Sarcomere Lengths in the Muscles of the Human Lower Limb." Journal of Anatomy 160:79-88. 
Davis, R. L., H. Weintraub, and A. B. Lassar. 1987. "Expression of a Single Transfected CDNA Converts Fibroblasts to Myoblasts.” Cell 51(6):987-1000. doi: 10.1016/00928674(87)90585-X.

Delfini, M. C., E. Hirsinger, O. Pourquié, and D. Duprez. 2000. "Delta 1-Activated Notch Inhibits Muscle Differentiation without Affecting Myf5 and Pax3 Expression in Chick Limb Myogenesis." Development (Cambridge, England) 127(23):5213-24.

Dunty, William C., Kristin K. Biris, Ravindra B. Chalamalasetty, Makoto M. Taketo, Mark Lewandoski, and Terry P. Yamaguchi. 2008. "Wnt3a/ $\beta$-Catenin Signaling Controls Posterior Body Development by Coordinating Mesoderm Formation and Segmentation." Development 135(1):85-94. doi: 10.1242/dev.009266.

Ehmsen, Jeffrey, Ellen Poon, and Kay Davies. 2002. "The Dystrophin-Associated Protein Complex." Journal of Cell Science 115(14):2801-3.

Eisenberg, Eli, and Erez Y. Levanon. 2013. "Human Housekeeping Genes, Revisited.” Trends in Genetics: TIG 29(10):569-74. doi: 10.1016/j.tig.2013.05.010.

Epstein, J. A., D. N. Shapiro, J. Cheng, P. Y. Lam, and R. L. Maas. 1996. "Pax3 Modulates Expression of the C-Met Receptor during Limb Muscle Development." Proceedings of the National Academy of Sciences of the United States of America 93(9):4213-18. doi: 10.1073/pnas.93.9.4213.

Fambrough, D. M. 1979. "Control of Acetylcholine Receptors in Skeletal Muscle.” Physiological Reviews. doi: 10.1152/physrev.1979.59.1.165.

Faustino Martins, Jorge-Miguel, Cornelius Fischer, Alessia Urzi, Ramon Vidal, Severine Kunz, Pierre-Louis Ruffault, Loreen Kabuss, Iris Hube, Elisabeta Gazzerro, Carmen Birchmeier, Simone Spuler, Sascha Sauer, and Mina Gouti. 2020. "Self-Organizing 3D Human Trunk Neuromuscular Organoids." Cell Stem Cell 26(2):172-186.e6. doi: 10.1016/j.stem.2019.12.007.

Frontera, Walter R., and Julien Ochala. 2015. "Skeletal Muscle: A Brief Review of Structure and Function.” Calcified Tissue International 96(3):183-95. doi: 10.1007/s00223-014-9915-y.

Gambke, B., G. E. Lyons, J. Haselgrove, A. M. Kelly, and N. A. Rubinstein. 1983. "Thyroidal and Neural Control of Myosin Transitions during Development of Rat Fast and Slow Muscles." FEBS Letters 156(2):335-39. doi: 10.1016/0014-5793(83)80524-9.

Garriock, Robert J., Ravindra B. Chalamalasetty, Mark W. Kennedy, Lauren C. Canizales, Mark Lewandoski, and Terry P. Yamaguchi. 2015. "Lineage Tracing of Neuromesodermal 
Progenitors Reveals Novel Wnt-Dependent Roles in Trunk Progenitor Cell Maintenance and Differentiation." Development 142(9):1628-38. doi: 10.1242/dev.111922.

Gattazzo, Francesca, Béatrice Laurent, Frédéric Relaix, Hélène Rouard, and Nathalie Didier. 2020. "Distinct Phases of Postnatal Skeletal Muscle Growth Govern the Progressive Establishment of Muscle Stem Cell Quiescence.” Stem Cell Reports 15(3):597-611. doi: 10.1016/j.stemcr.2020.07.011.

Gillies, Allison R., and Richard L. Lieber. 2011. "Structure and Function of the Skeletal Muscle Extracellular Matrix.” Muscle \& Nerve 44(3):318-31. doi: 10.1002/mus.22094.

Goldspink, G. 1970. “The Proliferation of Myofibrils during Muscle Fibre Growth.” Journal of Cell Science 6(2):593-603.

González, Aitor, Iris Manosalva, Tianxiao Liu, and Ryoichiro Kageyama. 2013. "Control of Hes7 Expression by Tbx6, the Wnt Pathway and the Chemical Gsk3 Inhibitor LiCl in the Mouse Segmentation Clock.” PLOS ONE 8(1):e53323. doi: 10.1371/journal.pone.0053323.

Grifone, Raphaelle, Josiane Demignon, Christophe Houbron, Evelyne Souil, Claire Niro, Mary J. Seller, Ghislaine Hamard, and Pascal Maire. 2005. "Six1 and Six4 Homeoproteins Are Required for Pax3 and Mrf Expression during Myogenesis in the Mouse Embryo." Development 132(9):2235-49. doi: 10.1242/dev.01773.

Gros, Jérôme, Marie Manceau, Virginie Thomé, and Christophe Marcelle. 2005. "A Common Somitic Origin for Embryonic Muscle Progenitors and Satellite Cells." Nature 435(7044):954-58. doi: 10.1038/nature03572.

Hagiwara, Nobuko, Michael Yeh, and Ann Liu. 2007. "Sox6 Is Required for Normal Fiber Type Differentiation of Fetal Skeletal Muscle in Mice." Developmental Dynamics: An Official Publication of the American Association of Anatomists 236(8):2062-76. doi: 10.1002/dvdy.21223.

Hellmuth, A. E., and D. B. Allbrook. 1971. "Muscle Satellite Cell Numbers during the Postnatal Period.” Journal of Anatomy 110(Pt 3):503.

Heslop, L., J. E. Morgan, and T. A. Partridge. 2000. "Evidence for a Myogenic Stem Cell That Is Exhausted in Dystrophic Muscle.” Journal of Cell Science 113 ( Pt 12):2299-2308.

Hirsinger, E., P. Malapert, J. Dubrulle, M. C. Delfini, D. Duprez, D. Henrique, D. Ish-Horowicz, and O. Pourquié. 2001. "Notch Signalling Acts in Postmitotic Avian Myogenic Cells to Control MyoD Activation.” Development (Cambridge, England) 128(1):107-16. 
Hirsinger, Estelle, Caroline Jouve, Julien Dubrulle, and Olivier Pourquié. 2000. "Somite Formation and Patterning." Pp. 1-65 in International Review of Cytology. Vol. 198. Academic Press.

Hubaud, Alexis, and Olivier Pourquié. 2014. "Signalling Dynamics in Vertebrate Segmentation." Nature Reviews Molecular Cell Biology 15(11):709-21. doi: 10.1038/nrm3891.

Janssen, I., S. B. Heymsfield, Z. M. Wang, and R. Ross. 2000. "Skeletal Muscle Mass and Distribution in 468 Men and Women Aged 18-88 Yr." Journal of Applied Physiology (Bethesda, Md.: 1985) 89(1):81-88. doi: 10.1152/jappl.2000.89.1.81.

Juhas, Mark, Nadia Abutaleb, Jason T. Wang, Jean Ye, Zohaib Shaikh, Chaichontat Sriworarat, Ying Qian, and Nenad Bursac. 2018. "Incorporation of Macrophages into Engineered Skeletal Muscle Enables Enhanced Muscle Regeneration." Nature Biomedical Engineering 2(12):942-54. doi: 10.1038/s41551-018-0290-2.

Juhas, Mark, George C. Engelmayr, Andrew N. Fontanella, Gregory M. Palmer, and Nenad Bursac. 2014. "Biomimetic Engineered Muscle with Capacity for Vascular Integration and Functional Maturation in Vivo." Proceedings of the National Academy of Sciences of the United States of America 111(15):5508-13. doi: 10.1073/pnas.1402723111.

Kablar, B., K. Krastel, C. Ying, A. Asakura, S. J. Tapscott, and M. A. Rudnicki. 1997. "MyoD and Myf-5 Differentially Regulate the Development of Limb versus Trunk Skeletal Muscle." Development (Cambridge, England) 124(23):4729-38.

Kassar-Duchossoy, Lina, Ellen Giacone, Barbara Gayraud-Morel, Aurélie Jory, Danielle Gomès, and Shahragim Tajbakhsh. 2005. "Pax3/Pax7 Mark a Novel Population of Primitive Myogenic Cells during Development." Genes \& Development 19(12):1426-31. doi: 10.1101/gad.345505.

Kim, Jaemin, Alessandro Magli, Sunny S. K. Chan, Vanessa K. P. Oliveira, Jianbo Wu, Radbod Darabi, Michael Kyba, and Rita C. R. Perlingeiro. 2017. "Expansion and Purification Are Critical for the Therapeutic Application of Pluripotent Stem Cell-Derived Myogenic Progenitors." Stem Cell Reports 9(1):12-22. doi: 10.1016/j.stemcr.2017.04.022.

Kume, Tsutomu, HaiYan Jiang, Jolanta M. Topczewska, and Brigid L. M. Hogan. 2001. "The Murine Winged Helix Transcription Factors, Foxc1 and Foxc2, Are Both Required for Cardiovascular Development and Somitogenesis." Genes \& Development 15(18):2470-82. doi: 10.1101/gad.907301.

Laclef, Christine, Ghislaine Hamard, Josiane Demignon, Evelyne Souil, Christophe Houbron, and Pascal Maire. 2003. "Altered Myogenesis in Six1-Deficient Mice." Development 130(10):2239-52. doi: 10.1242/dev.00440. 
Lapidos, Karen A., Rahul Kakkar, and Elizabeth M. McNally. 2004. "The Dystrophin Glycoprotein Complex: Signaling Strength and Integrity for the Sarcolemma." Circulation Research 94(8):1023-31. doi: 10.1161/01.RES.0000126574.61061.25.

Larsson, L., X. Li, A. Teresi, and G. Salviati. 1994. "Effects of Thyroid Hormone on Fast- and Slow-Twitch Skeletal Muscles in Young and Old Rats." The Journal of Physiology 481(1):149-61. doi: 10.1113/jphysiol.1994.sp020426.

Linke, Wolfgang A. 2018. "Titin Gene and Protein Functions in Passive and Active Muscle." Annual Review of Physiology 80:389-411. doi: 10.1146/annurev-physiol-021317-121234.

Liu, P., M. Wakamiya, M. J. Shea, U. Albrecht, R. R. Behringer, and A. Bradley. 1999. "Requirement for Wnt3 in Vertebrate Axis Formation." Nature Genetics 22(4):361-65. doi: 10.1038/11932.

Long, Chengzu, Hui Li, Malte Tiburcy, Cristina Rodriguez-Caycedo, Viktoriia Kyrychenko, Huanyu Zhou, Yu Zhang, Yi-Li Min, John M. Shelton, Pradeep P. A. Mammen, Norman Y. Liaw, Wolfram-Hubertus Zimmermann, Rhonda Bassel-Duby, Jay W. Schneider, and Eric N. Olson. 2018. "Correction of Diverse Muscular Dystrophy Mutations in Human Engineered Heart Muscle by Single-Site Genome Editing." Science Advances 4(1):eaap9004. doi: 10.1126/sciadv.aap9004.

Luz, M. a. M., M. J. Marques, and H. Santo Neto. 2002. "Impaired Regeneration of DystrophinDeficient Muscle Fibers Is Caused by Exhaustion of Myogenic Cells.” Brazilian Journal of Medical and Biological Research = Revista Brasileira De Pesquisas Medicas E Biologicas 35(6):691-95. doi: 10.1590/s0100-879x2002000600009.

M Ying, Ying, Wang S, Sang Y, Sun P, Lal B, Goodwin Cr, Guerrero-Cazares H, QuinonesHinojosa A, Laterra J, and Xia S. 2011. "Regulation of Glioblastoma Stem Cells by Retinoic Acid: Role for Notch Pathway Inhibition." Oncogene 30(31). Retrieved October 14, 2020 (https://pubmed.ncbi.nlm.nih.gov/21383690/).

Madden, Lauran, Mark Juhas, William E. Kraus, George A. Truskey, and Nenad Bursac. 2015. "Bioengineered Human Myobundles Mimic Clinical Responses of Skeletal Muscle to Drugs.” ELife 4:e04885. doi: 10.7554/eLife.04885.

Maffioletti, Sara Martina, Shilpita Sarcar, Alexander B. H. Henderson, Ingra Mannhardt, Luca Pinton, Louise Anne Moyle, Heather Steele-Stallard, Ornella Cappellari, Kim E. Wells, Giulia Ferrari, Jamie S. Mitchell, Giulia E. Tyzack, Vassilios N. Kotiadis, Moustafa Khedr, Martina Ragazzi, Weixin Wang, Michael R. Duchen, Rickie Patani, Peter S. Zammit, Dominic J. Wells, Thomas Eschenhagen, and Francesco Saverio Tedesco. 2018. "ThreeDimensional Human IPSC-Derived Artificial Skeletal Muscles Model Muscular 
Dystrophies and Enable Multilineage Tissue Engineering." Cell Reports 23(3):899-908. doi: 10.1016/j.celrep.2018.03.091.

Mankoo, Baljinder S., Susan Skuntz, Ian Harrigan, Elena Grigorieva, Al Candia, Christopher V. E. Wright, Heinz Arnheiter, and Vassilis Pachnis. 2003. "The Concerted Action of Meox Homeobox Genes Is Required Upstream of Genetic Pathways Essential for the Formation, Patterning and Differentiation of Somites." Development 130(19):4655-64. doi: 10.1242/dev.00687.

Marcelle, C., M. R. Stark, and M. Bronner-Fraser. 1997. “Coordinate Actions of BMPs, Wnts, Shh and Noggin Mediate Patterning of the Dorsal Somite.” Development 124(20):3955-63.

Martins, Gabriel G., Pedro Rifes, Rita Amândio, Gabriela Rodrigues, Isabel Palmeirim, and Sólveig Thorsteinsdóttir. 2009. "Dynamic 3D Cell Rearrangements Guided by a Fibronectin Matrix Underlie Somitogenesis." PLOS ONE 4(10):e7429. doi: 10.1371/journal.pone.0007429.

Mauro, Alexander. 1961. "SATELLITE CELL OF SKELETAL MUSCLE FIBERS.” The Journal of Biophysical and Biochemical Cytology 9(2):493-95.

Mayeuf-Louchart, A., M. Lagha, A. Danckaert, D. Rocancourt, F. Relaix, S. D. Vincent, and M. Buckingham. 2014. "Notch Regulation of Myogenic versus Endothelial Fates of Cells That Migrate from the Somite to the Limb." Proceedings of the National Academy of Sciences 111(24):8844-49. doi: 10.1073/pnas.1407606111.

Meech, Robyn, Katie N. Gonzalez, Marietta Barro, Anastasia Gromova, Lizhe Zhuang, Julie-Ann Hulin, and Helen P. Makarenkova. 2012. "Barx2 Is Expressed in Satellite Cells and Is Required for Normal Muscle Growth and Regeneration.” Stem Cells (Dayton, Ohio) 30(2):253-65. doi: 10.1002/stem.777.

Messina, Graziella, Stefano Biressi, Stefania Monteverde, Alessandro Magli, Marco Cassano, Laura Perani, Elena Roncaglia, Enrico Tagliafico, Linda Starnes, Christine E. Campbell, Milena Grossi, David J. Goldhamer, Richard M. Gronostajski, and Giulio Cossu. 2010. "Nfix Regulates Fetal-Specific Transcription in Developing Skeletal Muscle." Cell 140(4):554-66. doi: 10.1016/j.cell.2010.01.027.

Miller, K. J., D. Thaloor, S. Matteson, and G. K. Pavlath. 2000. "Hepatocyte Growth Factor Affects Satellite Cell Activation and Differentiation in Regenerating Skeletal Muscle." American Journal of Physiology. Cell Physiology 278(1):C174-181. doi: 10.1152/ajpcell.2000.278.1.C174. 
Missias, A. C., G. C. Chu, B. J. Klocke, J. R. Sanes, and J. P. Merlie. 1996. "Maturation of the Acetylcholine Receptor in Skeletal Muscle: Regulation of the AChR Gamma-to-Epsilon Switch." Developmental Biology 179(1):223-38. doi: 10.1006/dbio.1996.0253.

Miura, Shigeto, Shannon Davis, John Klingensmith, and Yuji Mishina. 2006. "BMP Signaling in the Epiblast Is Required for Proper Recruitment of the Prospective Paraxial Mesoderm and Development of the Somites." Development 133(19):3767-75. doi: 10.1242/dev.02552.

Montgomery, R. D. 1962. "Growth of Human Striated Muscle.” Nature 195(4837):194-95. doi: $10.1038 / 195194 \mathrm{a} 0$.

Moretti, A., L. Fonteyne, F. Giesert, P. Hoppmann, A. B. Meier, T. Bozoglu, A. Baehr, C. M. Schneider, D. Sinnecker, K. Klett, T. Fröhlich, F. Abdel Rahman, T. Haufe, S. Sun, V. Jurisch, B. Kessler, R. Hinkel, R. Dirschinger, E. Martens, C. Jilek, A. Graf, S. Krebs, G. Santamaria, M. Kurome, V. Zakhartchenko, B. Campbell, K. Voelse, A. Wolf, T. Ziegler, S. Reichert, S. Lee, F. Flenkenthaler, T. Dorn, I. Jeremias, H. Blum, A. Dendorfer, A. Schnieke, S. Krause, M. C. Walter, N. Klymiuk, K. L. Laugwitz, E. Wolf, W. Wurst, and C. Kupatt. 2020. "Somatic Gene Editing Ameliorates Skeletal and Cardiac Muscle Failure in Pig and Human Models of Duchenne Muscular Dystrophy." Nature Medicine 26(2):207-14. doi: 10.1038/s41591-019-0738-2.

Morimoto, Mitsuru, Yu Takahashi, Maho Endo, and Yumiko Saga. 2005. "The Mesp2 Transcription Factor Establishes Segmental Borders by Suppressing Notch Activity." Nature 435(7040):354-59. doi: 10.1038/nature03591.

Mourikis, Philippos, Ramkumar Sambasivan, David Castel, Pierre Rocheteau, Valentina Bizzarro, and Shahragim Tajbakhsh. 2012. "A Critical Requirement for Notch Signaling in Maintenance of the Quiescent Skeletal Muscle Stem Cell State." Stem Cells (Dayton, Ohio) 30(2):243-52. doi: 10.1002/stem.775.

Nam, Ki-Hwan, Alec S. T. Smith, Saifullah Lone, Sunghoon Kwon, and Deok-Ho Kim. 2015. "Biomimetic 3D Tissue Models for Advanced High-Throughput Drug Screening." Journal of Laboratory Automation 20(3):201-15. doi: 10.1177/2211068214557813.

Nassari, Sonya, Delphine Duprez, and Claire Fournier-Thibault. 2017. "Non-Myogenic Contribution to Muscle Development and Homeostasis: The Role of Connective Tissues." Frontiers in Cell and Developmental Biology 5:22. doi: 10.3389/fcell.2017.00022.

Naujok, Ortwin, Jana Lentes, Ulf Diekmann, Claudia Davenport, and Sigurd Lenzen. 2014. "Cytotoxicity and Activation of the Wnt/Beta-Catenin Pathway in Mouse Embryonic Stem Cells Treated with Four GSK3 Inhibitors.” BMC Research Notes 7:273. doi: 10.1186/17560500-7-273. 
Niro, Claire, Josiane Demignon, Stéphane Vincent, Yubing Liu, Julien Giordani, Nicolas Sgarioto, Maryline Favier, Isabelle Guillet-Deniau, Alexandre Blais, and Pascal Maire. 2010. "Six1 and Six4 Gene Expression Is Necessary to Activate the Fast-Type Muscle Gene Program in the Mouse Primary Myotome." Developmental Biology 338(2):168-82. doi: 10.1016/j.ydbio.2009.11.031.

Oginuma, Masayuki, Yasutaka Niwa, Deborah L. Chapman, and Yumiko Saga. 2008. "Mesp2 and Tbx6 Cooperatively Create Periodic Patterns Coupled with the Clock Machinery during Mouse Somitogenesis.” Development 135(15):2555-62. doi: 10.1242/dev.019877.

Olsen, Steen, Per Aagaard, Fawzi Kadi, Goran Tufekovic, Julien Verney, Jens L. Olesen, Charlotte Suetta, and Michael Kjær. 2006. "Creatine Supplementation Augments the Increase in Satellite Cell and Myonuclei Number in Human Skeletal Muscle Induced by Strength Training." The Journal of Physiology 573(Pt 2):525-34. doi: 10.1113/jphysiol.2006.107359.

Ostrovidov, Serge, Vahid Hosseini, Samad Ahadian, Toshinori Fujie, Selvakumar Prakash Parthiban, Murugan Ramalingam, Hojae Bae, Hirokazu Kaji, and Ali Khademhosseini. 2014. "Skeletal Muscle Tissue Engineering: Methods to Form Skeletal Myotubes and Their Applications." Tissue Engineering. Part B, Reviews 20(5):403-36. doi: 10.1089/ten.teb.2013.0534.

Parker, Maura H., Patrick Seale, and Michael A. Rudnicki. 2003. "Looking Back to the Embryo: Defining Transcriptional Networks in Adult Myogenesis." Nature Reviews Genetics 4(7):497-507. doi: 10.1038/nrg1109.

Petrof, B. J., J. B. Shrager, H. H. Stedman, A. M. Kelly, and H. L. Sweeney. 1993. "Dystrophin Protects the Sarcolemma from Stresses Developed during Muscle Contraction." Proceedings of the National Academy of Sciences of the United States of America 90(8):3710-14.

Poggesi, Corrado, Chiara Tesi, and Robert Stehle. 2005. "Sarcomeric Determinants of Striated Muscle Relaxation Kinetics." Pflugers Archiv: European Journal of Physiology 449(6):505-17. doi: 10.1007/s00424-004-1363-5.

Pourquié, Olivier. 2011. "Vertebrate Segmentation: From Cyclic Gene Networks to Scoliosis." Cell 145(5):650-63. doi: 10.1016/j.cell.2011.05.011.

Pourquié, Olivier, Ziad Al Tanoury, and Jérome Chal. 2018. "The Long Road to Making Muscle In Vitro." Pp. 123-42 in Current Topics in Developmental Biology. Vol. 129, Human Embryonic Stem Cells in Development, edited by A. H. Brivanlou. Academic Press. 
Racca, Alice W., Anita E. Beck, Vijay S. Rao, Galina V. Flint, Scott D. Lundy, Donald E. Born, Michael J. Bamshad, and Michael Regnier. 2013. "Contractility and Kinetics of Human Fetal and Human Adult Skeletal Muscle: Contractility and Kinetics of Human Fetal and Adult Skeletal Muscle." The Journal of Physiology 591(12):3049-61. doi: 10.1113/jphysiol.2013.252650.

Rajakumari, Sona, Jun Wu, Jeff Ishibashi, Hee-Woong Lim, An-Hoa Giang, Kyoung-Jae Won, Randall R. Reed, and Patrick Seale. 2013. "EBF2 Determines and Maintains Brown Adipocyte Identity." Cell Metabolism 17(4):562-74. doi: 10.1016/j.cmet.2013.01.015.

Ramkumar, Nitya, and Kathryn V. Anderson. 2011. "SnapShot: Mouse Primitive Streak." Cell 146(3):488-488.e2. doi: 10.1016/j.cell.2011.07.028.

Rao, Lingjun, Ying Qian, Alastair Khodabukus, Thomas Ribar, and Nenad Bursac. 2018. "Engineering Human Pluripotent Stem Cells into a Functional Skeletal Muscle Tissue." Nature Communications 9(1):126. doi: 10.1038/s41467-017-02636-4.

Relaix, Frédéric, Josiane Demignon, Christine Laclef, Julien Pujol, Marc Santolini, Claire Niro, Mounia Lagha, Didier Rocancourt, Margaret Buckingham, and Pascal Maire. 2013. "Six Homeoproteins Directly Activate Myod Expression in the Gene Regulatory Networks That Control Early Myogenesis." PLOS Genetics 9(4):e1003425. doi: 10.1371/journal.pgen.1003425.

Relaix, Frederic, and Peter S. Zammit. 2012. "Satellite Cells Are Essential for Skeletal Muscle Regeneration: The Cell on the Edge Returns Centre Stage." Development (Cambridge, England) 139(16):2845-56. doi: 10.1242/dev.069088.

Reubinoff, B. E., M. F. Pera, C. Y. Fong, A. Trounson, and A. Bongso. 2000. "Embryonic Stem Cell Lines from Human Blastocysts: Somatic Differentiation in Vitro." Nature Biotechnology 18(4):399-404. doi: 10.1038/74447.

Rios, Anne C., Olivier Serralbo, David Salgado, and Christophe Marcelle. 2011. "Neural Crest Regulates Myogenesis through the Transient Activation of NOTCH." Nature 473(7348):532-35. doi: 10.1038/nature09970.

Rosenblatt, J. David. 1992. "A Time Course Study of the Isometric Contractile Properties of Rat Extensor Digitorum Longus Muscle Injected with Bupivacaine." Comparative Biochemistry and Physiology Part A: Physiology 101(2):361-67. doi: 10.1016/03009629(92)90547-4.

Rossi, Giuliana, and Graziella Messina. 2014. "Comparative Myogenesis in Teleosts and Mammals." Cellular and Molecular Life Sciences 71(16):3081-99. doi: 10.1007/s00018014-1604-5. 
Saga, Y., N. Hata, H. Koseki, and M. M. Taketo. 1997. "Mesp2: A Novel Mouse Gene Expressed in the Presegmented Mesoderm and Essential for Segmentation Initiation." Genes \& Development 11(14):1827-39. doi: 10.1101/gad.11.14.1827.

Sambasivan, Ramkumar, Roseline Yao, Adrien Kissenpfennig, Laetitia Van Wittenberghe, Andràs Paldi, Barbara Gayraud-Morel, Hind Guenou, Bernard Malissen, Shahragim Tajbakhsh, and Anne Galy. 2011. "Pax7-Expressing Satellite Cells Are Indispensable for Adult Skeletal Muscle Regeneration." Development 138(17):3647-56. doi: 10.1242/dev.067587.

Schiaffino, S., S. Ausoni, L. Gorza, L. Saggin, K. Gundersen, and T. Lomo. 1988. "Myosin Heavy Chain Isoforms and Velocity of Shortening of Type 2 Skeletal Muscle Fibres." Acta Physiologica Scandinavica 134(4):575-76. doi: https://doi.org/10.1111/j.1365201X.1988.tb10643.x.

Schiaffino, Stefano, and Carlo Reggiani. 2011. "Fiber Types in Mammalian Skeletal Muscles." Physiological Reviews 91(4):1447-1531. doi: 10.1152/physrev.00031.2010.

Schiaffino, Stefano, Alberto C. Rossi, Vika Smerdu, Leslie A. Leinwand, and Carlo Reggiani. 2015. "Developmental Myosins: Expression Patterns and Functional Significance." Skeletal Muscle 5:22. doi: 10.1186/s13395-015-0046-6.

Schmeichel, Karen L., and Mina J. Bissell. 2003. "Modeling Tissue-Specific Signaling and Organ Function in Three Dimensions." Journal of Cell Science 116(Pt 12):2377-88. doi: $10.1242 /$ jcs.00503.

Schultz, Edward. 1974. "A Quantitative Study of the Satellite Cell Population in Postnatal Mouse Lumbrical Muscle.” The Anatomical Record 180(4):589-95. doi: 10.1002/ar.1091800405.

Seale, Patrick, Luc A. Sabourin, Adele Girgis-Gabardo, Ahmed Mansouri, Peter Gruss, and Michael A. Rudnicki. 2000. "Pax7 Is Required for the Specification of Myogenic Satellite Cells." Cell 102(6):777-86. doi: 10.1016/S0092-8674(00)00066-0.

Selvaraj, Sridhar, Ricardo Mondragon-Gonzalez, Bin Xu, Alessandro Magli, Hyunkee Kim, Jeanne Lainé, James Kiley, Holly Mckee, Fabrizio Rinaldi, Joy Aho, Nacira Tabti, Wei Shen, and Rita CR Perlingeiro. 2019. "Screening Identifies Small Molecules That Enhance the Maturation of Human Pluripotent Stem Cell-Derived Myotubes." ELife 8:e47970. doi: 10.7554/eLife.47970.

Shang, Min, Federica Cappellesso, Ricardo Amorim, Jens Serneels, Federico Virga, Guy Eelen, Stefania Carobbio, Melvin Y. Rincon, Pierre Maechler, Katrien De Bock, Ping-Chih Ho, Marco Sandri, Bart Ghesquière, Peter Carmeliet, Mario Di Matteo, Emanuele Berardi, and Massimiliano Mazzone. 2020. "Macrophage-Derived Glutamine Boosts Satellite Cells and Muscle Regeneration.” Nature 587(7835):626-31. doi: 10.1038/s41586-020-2857-9. 
Shansky, Janet, Joseph Chromiak, Michael Del Tatto, and Herman Vandenburgh. 1997. "A Simplified Method for Tissue Engineering Skeletal Muscle Organoids in Vitro." In Vitro Cellular \& Developmental Biology - Animal 33(9):659-61. doi: 10.1007/s11626-9970118-y.

Simonides, Warner S., and Cornelis van Hardeveld. 2008. "Thyroid Hormone as a Determinant of Metabolic and Contractile Phenotype of Skeletal Muscle." Thyroid: Official Journal of the American Thyroid Association 18(2):205-16. doi: 10.1089/thy.2007.0256.

Smith, Alec S. T., Jennifer Davis, Gabsang Lee, David L. Mack, and Deok-Ho Kim. 2016. "Muscular Dystrophy in a Dish: Engineered Human Skeletal Muscle Mimetics for Disease Modeling and Drug Discovery." Drug Discovery Today 21(9):1387-98. doi: 10.1016/j.drudis.2016.04.013.

Soong, Poh Loong, Malte Tiburcy, and Wolfram-Hubertus Zimmermann. 2012. "Cardiac Differentiation of Human Embryonic Stem Cells and Their Assembly into Engineered Heart Muscle." Current Protocols in Cell Biology Chapter 23:Unit23.8. doi: 10.1002/0471143030.cb2308s55.

Swist, Sandra, Andreas Unger, Yong Li, Anja Vöge, Marion von Frieling-Salewsky, Åsa Skärlén, Nicola Cacciani, Thomas Braun, Lars Larsson, and Wolfgang A. Linke. 2020. "Maintenance of Sarcomeric Integrity in Adult Muscle Cells Crucially Depends on Z-Disc Anchored Titin." Nature Communications 11(1):4479. doi: 10.1038/s41467-020-18131-2.

Tajbakhsh, Shahragim, and Ralf Spörle. 1998. "Somite Development: Constructing the Vertebrate Body.” Cell 92(1):9-16. doi: 10.1016/S0092-8674(00)80894-6.

Takemoto, Tatsuya, Masanori Uchikawa, Megumi Yoshida, Donald M. Bell, Robin Lovell-Badge, Virginia E. Papaioannou, and Hisato Kondoh. 2011. "Tbx6-Dependent Sox2 Regulation Determines Neural or Mesodermal Fate in Axial Stem Cells." Nature 470(7334):394-98. doi: 10.1038 /nature09729.

Tatsumi, Ryuichi, and Ronald E. Allen. 2004. "Active Hepatocyte Growth Factor Is Present in Skeletal Muscle Extracellular Matrix." Muscle \& Nerve 30(5):654-58. doi: 10.1002/mus.20114.

Tiburcy, Malte, James E. Hudson, Paul Balfanz, Susanne Schlick, Tim Meyer, Mei-Ling Chang Liao, Elif Levent, Farah Raad, Sebastian Zeidler, Edgar Wingender, Johannes Riegler, Mouer Wang, Joseph D. Gold, Izhak Kehat, Erich Wettwer, Ursula Ravens, Pieterjan Dierickx, Linda W. van Laake, Marie Jose Goumans, Sara Khadjeh, Karl Toischer, Gerd Hasenfuss, Larry A. Couture, Andreas Unger, Wolfgang A. Linke, Toshiyuki Araki, Benjamin Neel, Gordon Keller, Lior Gepstein, Joseph C. Wu, and Wolfram-Hubertus 
Zimmermann. 2017. "Defined Engineered Human Myocardium With Advanced Maturation for Applications in Heart Failure Modeling and Repair." Circulation 135(19):1832-47. doi: 10.1161/CIRCULATIONAHA.116.024145.

Tiburcy, Malte, Alex Markov, Lena K. Kraemer, Peter Christalla, Margret Rave-Fraenk, Henrike J. Fischer, Holger M. Reichardt, and Wolfram-Hubertus Zimmermann. 2019. "Regeneration Competent Satellite Cell Niches in Rat Engineered Skeletal Muscle." FASEB BioAdvances 1(12):731-46. doi: 10.1096/fba.2019-00013.

Tiburcy, Malte, Tim Meyer, Poh Loong Soong, and Wolfram-Hubertus Zimmermann. 2014. "Collagen-Based Engineered Heart Muscle." Pp. 167-76 in Cardiac Tissue Engineering: Methods and Protocols, Methods in Molecular Biology, edited by M. Radisic and L. D. Black III. New York, NY: Springer.

Uezumi, Akiyoshi, So-ichiro Fukada, Naoki Yamamoto, Shin'ichi Takeda, and Kunihiro Tsuchida. 2010. "Mesenchymal Progenitors Distinct from Satellite Cells Contribute to Ectopic Fat Cell Formation in Skeletal Muscle." Nature Cell Biology 12(2):143-52. doi: 10.1038/ncb2014.

Vandenburgh, H. H., P. Karlisch, and L. Farr. 1988. "Maintenance of Highly Contractile TissueCultured Avian Skeletal Myotubes in Collagen Gel." In Vitro Cellular \& Developmental Biology: Journal of the Tissue Culture Association 24(3):166-74. doi: 10.1007/BF02623542.

van der Velden, Jos L. J., Ramon C. J. Langen, Marco C. J. M. Kelders, Emiel F. M. Wouters, Yvonne M. W. Janssen-Heininger, and Annemie M. W. J. Schols. 2006. "Inhibition of Glycogen Synthase Kinase-3 $\beta$ Activity Is Sufficient to Stimulate Myogenic Differentiation." American Journal of Physiology-Cell Physiology 290(2):C453-62. doi: 10.1152/ajpcell.00068.2005.

Volonte, Daniela, Youhua Liu, and Ferruccio Galbiati. 2005. "The Modulation of Caveolin-1 Expression Controls Satellite Cell Activation during Muscle Repair." FASEB Journal: Official Publication of the Federation of American Societies for Experimental Biology 19(2):237-39. doi: 10.1096/fj.04-2215fje.

Wakeford, S., D. J. Watt, and T. A. Partridge. 1991. "X-Irradiation Improves Mdx Mouse Muscle as a Model of Myofiber Loss in DMD." Muscle \& Nerve 14(1):42-50. doi: 10.1002/mus.880140108.

White, Robert B., Anne-Sophie Biérinx, Viola F. Gnocchi, and Peter S. Zammit. 2010. "Dynamics of Muscle Fibre Growth during Postnatal Mouse Development." BMC Developmental Biology 10(1):21. doi: 10.1186/1471-213X-10-21. 
Willoughby, D. S., and J. Rosene. 2001. "Effects of Oral Creatine and Resistance Training on Myosin Heavy Chain Expression." Medicine and Science in Sports and Exercise 33(10):1674-81. doi: 10.1097/00005768-200110000-00010.

Wolf, F. Alexander, Philipp Angerer, and Fabian J. Theis. 2018. "SCANPY: Large-Scale SingleCell Gene Expression Data Analysis.” Genome Biology 19(1):15. doi: 10.1186/s13059017-1382-0.

van der Worp, H. Bart, David W. Howells, Emily S. Sena, Michelle J. Porritt, Sarah Rewell, Victoria O'Collins, and Malcolm R. Macleod. 2010. "Can Animal Models of Disease Reliably Inform Human Studies?" PLoS Medicine 7(3). doi: 10.1371/journal.pmed.1000245.

Xi, Haibin, Wakana Fujiwara, Karen Gonzalez, Majib Jan, Simone Liebscher, Ben Van Handel, Katja Schenke-Layland, and April D. Pyle. 2017. "In Vivo Human Somitogenesis Guides Somite Development from HPSCs." Cell Reports 18(6):1573-85. doi: 10.1016/j.celrep.2017.01.040.

Xi, Haibin, Justin Langerman, Shan Sabri, Peggie Chien, Courtney S. Young, Shahab Younesi, Michael Hicks, Karen Gonzalez, Wakana Fujiwara, Julia Marzi, Simone Liebscher, Melissa Spencer, Ben Van Handel, Denis Evseenko, Katja Schenke-Layland, Kathrin Plath, and April D. Pyle. 2020. "A Human Skeletal Muscle Atlas Identifies the Trajectories of Stem and Progenitor Cells across Development and from Human Pluripotent Stem Cells." Cell Stem Cell 27(1):158-176.e10. doi: 10.1016/j.stem.2020.04.017.

Xu, Bin, Mengen Zhang, Rita C. R. Perlingeiro, and Wei Shen. 2019. "Skeletal Muscle Constructs Engineered from Human Embryonic Stem Cell Derived Myogenic Progenitors Exhibit Enhanced Contractile Forces When Differentiated in a Medium Containing EGM-2 Supplements." Advanced Biosystems 3(12):1900005. doi: 10.1002/adbi.201900005.

Yablonka-Reuveni, Zipora. 2011. "The Skeletal Muscle Satellite Cell: Still Young and Fascinating at 50." The Journal of Histochemistry and Cytochemistry: Official Journal of the Histochemistry Society 59(12):1041-59. doi: 10.1369/0022155411426780.

Yamaguchi, T. P., K. Harpal, M. Henkemeyer, and J. Rossant. 1994. "Fgfr-1 Is Required for Embryonic Growth and Mesodermal Patterning during Mouse Gastrulation." Genes \& Development 8(24):3032-44. doi: 10.1101/gad.8.24.3032.

Yin, Hang, Feodor Price, and Michael A. Rudnicki. 2013. "Satellite Cells and the Muscle Stem Cell Niche." Physiological Reviews 93(1):23-67. doi: 10.1152/physrev.00043.2011. 
Yoon, Jeong Kyo, Randall T. Moon, and Barbara Wold. 2000. "The BHLH Class Protein PMesogenin1 Can Specify Paraxial Mesoderm Phenotypes." Developmental Biology 222(2):376-91. doi: 10.1006/dbio.2000.9717.

Zimmermann, W. H., C. Fink, D. Kralisch, U. Remmers, J. Weil, and T. Eschenhagen. 2000. "Three-Dimensional Engineered Heart Tissue from Neonatal Rat Cardiac Myocytes." Biotechnology and Bioengineering 68(1):106-14. 


\section{Appendix}

\subsection{Reagents}

Table A1. List of Chemicals, Peptides and Recombinant Proteins

\begin{tabular}{|c|c|c|}
\hline $\begin{array}{l}\text { Chemicals, Peptides and Recombinant } \\
\text { Proteins }\end{array}$ & Source & Identifier \\
\hline Aceton & Carl Roth & Cat. $\# 5025$ \\
\hline Agarose, universal, peqGOLD & VWR Peqlab & Cat. \#7322789P \\
\hline B-27 Supplement & $\begin{array}{ll}\text { Thermo } \quad \text { Fisher } \\
\text { Scientific }\end{array}$ & Cat. \#17504044 \\
\hline Bovine Serum Albumin & Sigma-Aldrich & Cat. \#A3294 \\
\hline Carbachol (Carbamoyl choline chloride) & Sigma-Aldrich & Cat. \#C4382 \\
\hline $\begin{array}{l}\text { CHIR99021, 6-[[2-[[4-(2,4-Dichlorphenyl)-5-(5- } \\
\text { methyl-1H-imidazol-2-yl)-2 } \\
\text { pyrimidinyl]amino]ethyl]amino]-3 } \\
\text { pyridinecarbonitril, CT99021 }\end{array}$ & Stemgent & Cat. $\# 040004$ \\
\hline Collagen (Acid Solubilized Telo Collagen) & $\begin{array}{ll}\text { LLC } & \text { Collagen } \\
\text { Solutions } & \end{array}$ & Cat. \#FS22024 \\
\hline Creatine monohydrate & Sigma-Aldrich & Cat. \#C3630 \\
\hline Cardiotoxin & Latoxan & Cat. \#L8102 \\
\hline $\begin{array}{l}\text { DAPT, tert-Butyl }(2 S)-2-[[(2 S)-2-[[2-(3,5- \\
\text { difluorophenyl)acetyl]amino]propanoyl] }] \text { amino]- } \\
\text { 2-phenylacetate Gamma } \gamma \text {-secretase inhibitor }\end{array}$ & Tocris & Cat. \#2634 \\
\hline Dimethyl sulfoxide (DMSO) & Sigma-Aldrich & Cat. \#D2650 \\
\hline $\begin{array}{l}\text { DMEM, low glucose, GlutaMAX }{ }^{\mathrm{TM}} \text { Supplement, } \\
\text { pyruvate }\end{array}$ & $\begin{array}{ll}\text { Thermo } & \text { Fisher } \\
\text { Scientific } & \end{array}$ & Cat. \#10567014 \\
\hline
\end{tabular}




\begin{tabular}{|c|c|c|}
\hline DMEM, powder,low glucose, pyruvate & $\begin{array}{ll}\text { Thermo } & \text { Fisher } \\
\text { Scientific } & \end{array}$ & Cat. \#31600036 \\
\hline DNase I recombinant, RNase-free & Roche & Cat. $\# 4716728001$ \\
\hline Ethanol $(99.5 \%)$ & Carl Roth & Cat. $\# 5054$ \\
\hline Fast SYBR Green Master Mix & $\begin{array}{ll}\text { Thermo } & \text { Fisher } \\
\text { Scientific } & \end{array}$ & Cat. $\# 4385610$ \\
\hline FemtoLUCENT ${ }^{\mathrm{TM}}$ Plus HRP & Gbiosciences & Cat. \#786003 \\
\hline FGF-2, basic fibroblast growth factor & Peprotech & Cat. \#AF10018B \\
\hline Fluoromount-G & Southern Biotech & Cat. $\# 010001$ \\
\hline Formalin solution $4 \%$ (Histofix) & Carl Roth & Cat. \#P0871 \\
\hline HGF, Hepatocyte growth factor & Peprotech & Cat. \#10039B \\
\hline High Capacity cDNA Reverse Transcription Kit & Applied Biosystems & Cat. \#10400745 \\
\hline Knockout serum replacement & $\begin{array}{ll}\text { Thermo } & \text { Fisher } \\
\text { Scientific } & \end{array}$ & Cat. \#10828028 \\
\hline $\begin{array}{l}\text { LDN193189, 4-[6-(4-piperazin-1- } \\
\text { ylphenyl)pyrazolo[1,5-a]pyrimidin-3- } \\
\text { yl]quinoline;hydrochloride }\end{array}$ & Stemgent & Cat. $\# 040074$ \\
\hline Matrigel (Growth Factor Reduced) & $\begin{array}{l}\text { Becton Dickinson/BD } \\
\text { Biosciences }\end{array}$ & Cat. $\# 354230$ \\
\hline MEM Non-essential amino acid solution, 100x & $\begin{array}{ll}\text { Thermo } & \text { Fisher } \\
\text { Scientific } & \end{array}$ & Cat. \#11140035 \\
\hline 2-Mercaptoethanol & $\begin{array}{ll}\text { Thermo } & \text { Fisher } \\
\text { Scientific } & \end{array}$ & Cat. \#21985023 \\
\hline Mini-PROTEIN TGX ${ }^{\mathrm{TM}}$ Precast gels & BioRad & Cat. \#4561084 \\
\hline N-2 Supplement & $\begin{array}{ll}\text { Thermo } & \text { Fisher } \\
\text { Scientific } & \end{array}$ & Cat. \#17502048 \\
\hline $\mathrm{NaOH} 1 \mathrm{~mol} / \mathrm{L}(1 \mathrm{~N})$ & Carl Roth & Cat. \#K0211 \\
\hline Phosphate-buffered saline (PBS) & $\begin{array}{ll}\text { Thermo } & \text { Fisher } \\
\text { Scientific } & \\
\end{array}$ & Cat. \#14190094 \\
\hline
\end{tabular}




\begin{tabular}{|c|c|c|}
\hline Penicillin/Streptomycin (100x) & $\begin{array}{l}\text { Thermo } \quad \text { Fisher } \\
\text { Scientific }\end{array}$ & Cat. \#15140122 \\
\hline Phosphatase inhibitor, PhosSTOP & Roche & Cat. \#4906845001 \\
\hline SYLGARD $^{\mathrm{TM}} 184$ Silicone Elastomer Kit & Dow corning & Cat. \#1317318 \\
\hline Protease inhibitor, cOmplete Mini & Roche & Cat. \#11836153001 \\
\hline StemMACS iPS-Brew XF Medium & Miltenyi Biotec & Cat. \#130104368 \\
\hline Triiodo-L-thyronine (T3) & Sigma-Aldrich & Cat. \#T2877 \\
\hline Triton $^{\mathrm{TM}} \mathrm{X}-100$ & Sigma-Aldrich & Cat. \#T8787 \\
\hline Trizol $^{\mathrm{TM}}$ Reagent & $\begin{array}{ll}\text { Thermo } & \text { Fisher } \\
\text { Scientific } & \end{array}$ & Cat. $\# 15596026$ \\
\hline TrypLE Express & $\begin{array}{ll}\text { Thermo } & \text { Fisher } \\
\text { Scientific } & \end{array}$ & Cat. $\# 12605010$ \\
\hline Versene Solution (0.48 mM EDTA) & $\begin{array}{ll}\text { Thermo } & \text { Fisher } \\
\text { Scientific } & \end{array}$ & Cat. $\# 15040033$ \\
\hline $\begin{array}{l}\text { Y27632, 4-[(1R)-1-aminoethyl]-N-pyridin-4- } \\
\text { ylcyclohexane-1-carboxamide;dihydrochloride }\end{array}$ & Stemgent & Cat. $\# 04001210$ \\
\hline
\end{tabular}


Table A2. List of antibodies

\begin{tabular}{|l|l|l|}
\hline Antibodies & Source & Identifier \\
\hline$\alpha$-actinin (Sarcomeric) & Sigma-Aldrich & A7811 \\
\hline Beta-Dystroglycan & Leica Biosystem & NCL-b-DG \\
\hline Dystrophin Monoclonal & Millipore & MAB 1645 \\
\hline Dystrophin Monoclonal & Sigma-Aldrich & MANDYS8 \\
\hline Goat anti-mouse Alexa Fluor 488 & Thermo Fisher Scientific & A32723 \\
\hline Goat anti-mouse Alexa Fluor 546 & Thermo Fisher Scientific & A-11030 \\
\hline Goat anti-rabbit Alexa Fluor 546 & Thermo Fisher Scientific & A-11035 \\
\hline HRP conjugated goat anti-mouse-IgG & Dako & P0161 \\
\hline Ki67 & Abcam & Ab15580 \\
\hline Laminin & Sigma-Aldrich & L 9393 \\
\hline MyoD & Dako & MA1-41017 \\
\hline Myogenin & DSHB & F5D-c \\
\hline Myosin heavy chain 2 (fast type) & DSHB & A4.74 \\
\hline Myosin heavy chain 3 (embryonic) & DSHB & F1.652 \\
\hline Myosin heavy chain 7 (slow type) & DSHB & A4.951 \\
\hline Neurofilament H, SMI32 & Biolegend & SMI-32P \\
\hline Oct4 & Abcam & Ab19857 \\
\hline Pax3 & DSHB & Pax3-c \\
\hline Pax7 & DSHB & Pax7-c \\
\hline Vinculin (VCL) & Sigma-Aldrich & V9264 \\
\hline
\end{tabular}




\subsection{Gene panel}

\section{List of muscle enriched genes (muscle_gene panel)}

ABCC9, ABCG1, ABLIM3, ACADL, ACOT11, ACTC1, ACTN2, ACVR2A, ADAM2, ADAMTS20, ADAMTS5, ADGRA1, AFAP1L1, AKR1C1, AKR1C2, AKR1C3, AL138752.2, AL591806.4, ALPK2, ALPK3, AP006333.1, ARHGAP18, ARHGAP9, ARMC3, ARPP21, ASB5, ATP2A1, ATP2B1, B4GALNT3, BBOX1, BCL11B, BICDL1, BLCAP, BMPR1B, BVES, C10ORF90, C1QTNF7, CACNA1S, CACNA2D1, CACNA2D4, CAP2, CASQ2, CASS4, CASZ1, CCDC141, CCNG2, CD82, CD96, CDH15, CDH7, CDK15, CELF2, CFL2, CHD7, CHODL, CHRNA1, CHRNB1, CHRND, CHRNG, CLCN5, CLSTN2, COBL, COL19A1, COL25A1, COQ8A, CPM, CYB5R1, DACH1, DES, DGKB, DLG2, DNAH17, DOCK6, DOCK9, DOK7, DSCAML1, DUSP10, DUSP27, DYSF, ECHDC2, EFHD2, EHBP1L1, EMC10, ENO3, ENPP1, ERBB3, EYA1, EYA2, EYA4, EZR, FASTKD1, FAT1, FGD4, FGF10, FGF13, FGF9, FGFR4, FNDC5, FOXO1, FOXP2, FREM2, FRMD3, FRMPD1, FST, FSTL4, GABRB3, GADL1, GATM, GCNT1, GEN1, GLI1, GLRB, GPA33, GPRIN3, GRAMD1B, GREM2, GSG1L, HES, HEYL, HIPK4, HS6ST2, IGDCC4, INPP4, ITGA4, ITGA7, ITGB6, ITIH5, JAM3, JPH1, JPH2, JSRP1, KCND3, KCNN2, KIF24, KLHL13, KLHL14, KLHL31, KLHL41, KREMEN1, KREMEN2, LDB3, LDLRAD3, LFNG, LHFPL6, LINC00514, LMOD3, LRIG1, LRP5, LRRC3B, LRRFIP1, LZTS1, MACROD1, MAMSTR, MAN1C1, MAP4K1, MARCHF3, MATR3_2, MB21D2, MEF2C, MEGF10, MET, MICAL1, MICAL2, MLIP, MMP23B, MRLN, MSR1, MTHFD1L, MYH3, MYL1, MYL4, MYLK4, MYO18B, MYOG, MYOM3, MYOZ2, MYPN, NCOA1, NEB, NECTIN1, NES, NEXN, NKAIN4, NNAT, NPNT, NRK, NTF3, NTN5, NXPH2, OLFML2A, OLFML2B, ORC4, OVCH1, P3H2, PALM2AKAP2, PALMD, PARM1, PAX7, PC, PDE1C, PDGFC, PDLIM3, PITPNM3, PITX2, PITX3, PKP4, PLAC1, PLS3, PLXNA2, POLA1, PPFIA4, PRELID3A, PRKCB, PRUNE2, PSEN2, PTGFR, PUS7, RALYL, RAPSN, RASSF3, RASSF4, RBM20, RBM24, RCL1, RELL1, RGS7, RIF1, RYR1, RYR2, SCN7A, SEMA3D, SEMA6B, SEPTIN4, SETD7, SGCA, SGCD, SHD, SHISA9, SIM1, SKP2, SLC16A10, SLC24A2, SLC24A3, SLC38A5, SLC7A2, SLC8A3, SLF2, SMC6, SMOC1, SMYD1, SNTG2, SOX6, SPAG6, SPATS2L, SRL, SRPK3, ST6GALNAC5, ST7, STARD13, STC1, STC2, STK26, SYN2, SYNE3, SYTL3, TANC1, TEAD4, TMEM131L, TMEM232, TMTC1, TNC, TNNI1, TNNT1, TNNT2, TNNT3, TNPO1, TPM2, TRDN, TRIM55, TRIM72, TRPA1, TSHZ3, TSPAN12, TSPAN33, UNC45B, USP6, VGLL2, VGLL3, VWCE, WDR43, ZEB2, ZNF536 


\subsection{Supplemental figure}

\section{Supplemental Figure 1}
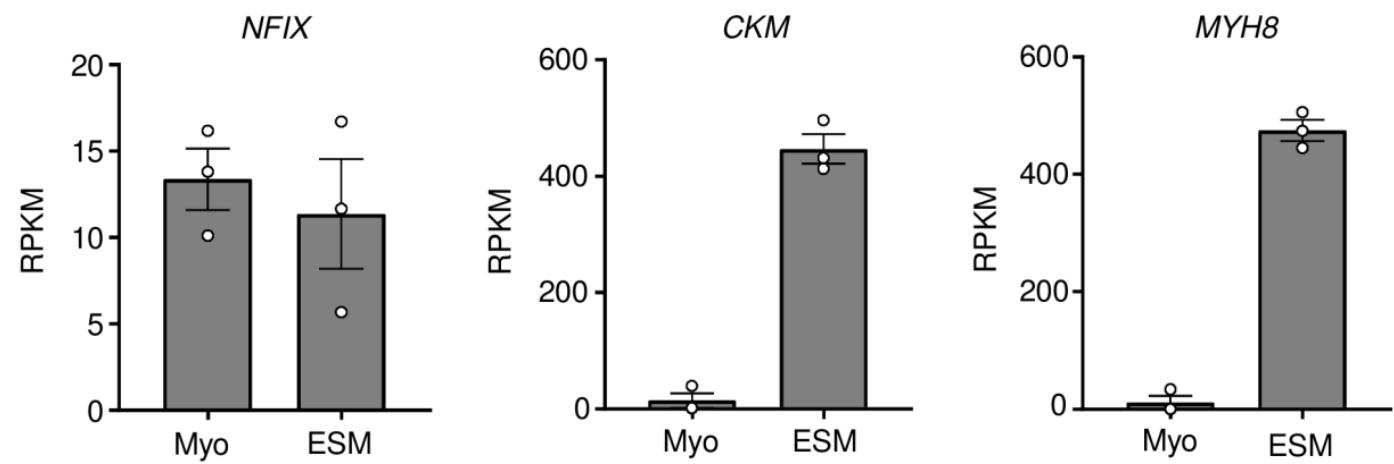

Supplemental Figure 1. Secondary myogenesis in ESM.

Expression levels (Reads per Kilobase Million, RPKM) of genes indicative of secondary myogenesis in day 22 monolayer skeletal muscle cultures (Myo) and 5 wk old ESM generated from hPSC. 